\title{
Time-dependent non-reflecting boundary conditions for simulations of wave propagation in geophysical shear flows
}

\section{Nijimbere Victor}

\author{
A thesis submitted to \\ the Faculty of Graduate Studies and Research \\ in partial fulfillment of \\ the requirements for the degree of \\ Master of Science
}

School of Mathematics and Statistics
Ottawa-Carleton Institute of Mathematics and Statistics

Carleton University

Ottawa, Ontario, Canada

March, 2010

Copyright (C)

2010 Nijimbere Victor 


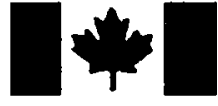

Library and Archives

Canada

Published Heritage

Branch

395 Wellington Street

Ottawa ON K1A ON4

Canada
Bibliotheque et

Archives Canada

Direction du

Patrimoine de l'édition

395, rue Wellington

Ottawa ON K1A ON4

Canada
Your file Votre référence
ISBN: 978-0-494-68619-5
Our file Notre référence
ISBN: $978-0-494-68619-5$

\section{NOTICE:}

The author has granted a nonexclusive license allowing Library and Archives Canada to reproduce, publish, archive, preserve, conserve, communicate to the public by telecommunication or on the Internet, loan, distribute and sell theses worldwide, for commercial or noncommercial purposes, in microform, paper, electronic and/or any other formats.

The author retains copyright ownership and moral rights in this thesis. Neither the thesis nor substantial extracts from it may be printed or otherwise reproduced without the author's permission.
AVIS:

L'auteur a accordé une licence non exclusive permettant à la Bibliothèque et Archives Canada de reproduire, publier, archiver, sauvegarder, conserver, transmettre au public par télécommunication ou par l'Internet, prêter, distribuer et vendre des thèses partout dans le monde, à des fins commerciales ou autres, sur support microforme, papier, électronique et/ou autres formats.

L'auteur conserve la propriété du droit d'auteur et des droits moraux qui protège cette thèse. Ni la thèse ni des extraits substantiels de celle-ci ne doivent être imprimés ou autrement reproduits sans son autorisation.
In compliance with the Canadian Privacy Act some supporting forms may have been removed from this thesis.

While these forms may be included in the document page count, their removal does not represent any loss of content from the thesis.
Conformément à la loi canadienne sur la protection de la vie privée, quelques formulaires secondaires ont été enlevés de cette thèse.

Bien que ces formulaires aient inclus dans la pagination, il n'y aura aucun contenu manquant. 


\begin{abstract}
In numerical simulations of wave propagation in fluids, one often needs to apply non-reflecting boundary conditions or radiation conditions at the outflow boundary to prevent wave reflections. This thesis examines time-dependent radiation conditions for modeling the propagation of atmospheric gravity waves and Rossby waves.

The radiation condition is written in terms of a Laplace convolution integral, so it is non-local in time and expensive to compute, requiring values of the dependent variable at all previous time levels. The convolution integral is approximated so as to make the computations local in time and hence less expensive.

The results are compared with those obtained by simply setting the fluid variables to zero at boundary or applying a steady radiation condition and are found to be more stable and to more accurately represent the exact solutions. A nonlinear correction to the time-dependent radiation condition is also developed and implemented.
\end{abstract}




\section{Acknowledgements}

I would like to thank my supervisor, Prof. Lucy Campbell for her patience, wisdom, kindness, for her excellent professionalism in teaching and specially for being an excellent guider in research throughout my Master's thesis work at Carleton University in the School of Mathematics and Statistics.

I would like also to thank my parents, my brother and my sister and their families and all my friends for their moral support. I would like to thank Edmond Nkinzo Kabushemeye for helping me to make the movies which were helpful in the analysis of the results presented in this thesis work. 


\section{Contents}

Abstract

$\begin{array}{lll}\text { Acknowledgements } & \text { ii }\end{array}$

List of figures vii

List of symbols $\quad$ xv

1 Introduction 1

1.1 Wave simulations in geophysical fluids $\ldots \ldots \ldots \ldots \ldots$

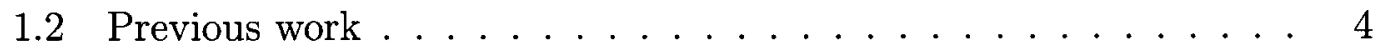

1.3 An overview of the thesis . . . . . . . . . . 7

2 Governing equations $\quad 11$

2.1 The basic equations of fluid dynamics . . . . . . . . . . . 11

2.1.1 The Navier-Stokes equations . . . . . . . . . . . . . 12

2.1 .2 The continuity equation $\ldots \ldots \ldots \ldots 14$

2.1 .3 The energy equation $\ldots \ldots \ldots \ldots \ldots$

2.2 The governing equations for Rossby waves $\ldots \ldots \ldots \ldots$

2.2.1 The beta plane approximation and the Coriolis force $\ldots 19$

2.2 .2 The barotropic vorticity equation $\ldots \ldots \ldots \ldots$ iii 
2.2.3 The non-dimensional barotropic vorticity equation . . . . . 22

2.3 Governing equations for gravity waves . . . . . . . . . 25

2.3.1 The Momentum equation under the Boussinesq approxi-

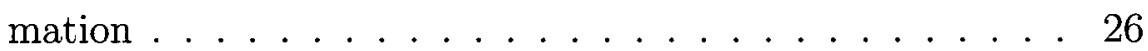

2.3.2 The energy equation under the Boussinesq approximation . 29

2.3.3 The nondimensional equations for $\Psi$ and $\varrho \ldots . . . . .30$

2.4 Asymptotic behavior of solutions of the linear problems across the critical layer ....................... 33

2.5 Exact expressions for transient Rossby and gravity waves . . . . . 37

3 The derivation of the radiation conditions $\quad 40$

3.1 Steady radiation condition . . . . . . . . . . . . . . 41

3.1.1 Steady radiation condition for Rossby waves . . . . . . . . 41

3.1.2 Steady radiation condition for gravity waves . . . . . . . . 44

3.2 Time-dependent linear radiation conditions . . . . . . . . . . 47

3.2.1 Time-dependent linear radiation condition for Rossby waves 47

3.2.2 Time-dependent linear radiation condition for gravity waves 50

3.3 Time-dependent nonlinear radiation conditions . . . . . . . . . 53

4 Numerical implementation $\quad 60$

4.1 Local in time implementation of the linear time-dependent radiation condition .................. 69

4.2 Implementation of the nonlinear correction . . . . . . . . . 72

5 Results of the numerical simulations $\quad 74$

5.1 Results of the simulations of the Rossby propagation . . . . . . 75 
5.1.1 Results of the simulations in a configuration with a critical layer in the flow ................ 75

5.1.2 Results of simulations of Rossby wave propagations in a configuration without a critical layer in flow . . . . . 80

5.1.3 The results of nonlinear simulations . . . . . . . . 88

5.2 The results of simulations of gravity wave propagation . . . . . 101

5.2.1 The results of simulations of gravity wave propagations in a configuration with a critical layer in the flow . . . . . 103

5.2.2 Results of simulations of gravity wave propagations in a configuration without a critical layer in flow . . . . . 106

5.2.3 The results of nonlinear simulations . . . . . . . . 113

6 Conclusions

A Dispersion relation

A.1 Rossby wave dispersion relation . . . . . . . . . . . . . . 129

A.2 Gravity wave dispersion relation . . . . . . . . . . . 130

B The Eliassen-Palm theorem and the mean flow evolution

B.1 The nonlinear evolution of the mean flow . . . . . . . . . . . 131

B.2 The Eliassen-Palm theorem ... . . . . . . . . . . 134

C The Richardson number and Miles-Howard theorem

D Exact solution for transient Rossby waves in the long-wave limit 140

D.1 Convergence of the solution . . . . . . . . . . . . . . 145 


\section{List of Figures}

5.1 Simulation of Rossby wave propagation: the background flow speed is $\bar{u}=\tanh y$ as a function of the latitude $y$. The critical level is at $y_{c}=0 \ldots \ldots \ldots \ldots \ldots$

5.2 Linear and nonlinear simulations of Rossby wave propagation: contour plot of $\Psi(x, y, t)$ at $t=70,(a)$ linear simulation with $\epsilon=0$ and $(b)$ nonlinear simulation with $\epsilon=0.05$. In both $(a)$ and (b) $\delta=0.2$ and $\beta=1$. A periodic forcing $e^{i k_{o} x}, k_{o}=2$, has been applied at the upper boundary of the rectangular domain. The background flow speed is $\bar{u}=\tanh y$, the critical level is at $y=0$. Both the contour show that there are not much transmission activity beyond the critical layer. . . . . . . . . . 78 
5.3 Linear and nonlinear simulations of Rossby wave propagation: plot of the jump in the momentum flux $[F]$ across the critical layer as a function of time for $0 \leq t \leq 70$. A periodic forcing $e^{i k_{o} x}, k_{o}=2$, has been applied at the upper boundary of the rectangular domain, $\delta=0.2$ and $\beta=1$. The background flow speed is $\bar{u}=\tanh y$, the critical level is at $y=0$. The dashed line shows the result obtained for the linear simulation $(\varepsilon=0)$ while the solid line shows the result obtained for the nonlinear simulation $(\varepsilon=0.05)$. In the linear case $[F]$ becomes almost constant by about $t=20$. This indicates absorption of the waves by the mean flow. In the nonlinear case $[F]$ alternates between positive values (absorption) and the negative values (overreflection). . . . . . 79

5.4 Linear and nonlinear simulations of Rossby wave propagation: plot of the momentum flux as function of the latitude $y$ at the time $t=70$. A periodic forcing $e^{i k_{o} x}, k_{o}=2$, has been applied at the upper boundary of the rectangular domain, $\delta=0.2$ and $\beta=1$. The background flow speed is $\bar{u}=\tanh y$, the critical level is at $y=0$. The dashed line corresponds to the linear simulation $(\varepsilon=0)$ while the solid line corresponds to the nonlinear simulation $(\varepsilon=0.05) \ldots \ldots \ldots \ldots \ldots \ldots$ 
5.5 Linear simulation of Rossby wave propagation $(\varepsilon=0)$ with the zero boundary condition: contour plots of the streamfunction $\psi(x, y, t)$ obtained at (a) $t=2$, (b) $t=5$, (c) $t=10$, (d) $t=23$, (e) $t=42$ and (f) $t=62$. A periodic forcing $e^{i k_{o} x}, k_{o}=2$, has been applied at the upper boundary of the rectangular domain, $\delta=0.2$ and $\beta=1$. The background flow is constant, $\bar{u}=1 \ldots$. . 83

5.6 Linear simulation of Rossby wave propagation $(\varepsilon=0)$ with the steady boundary condition: contour plots of the streamfunction $\psi(x, y, t)$ obtained at (a) $t=2$, (b) $t=5$, (c) $t=10$, (d) $t=23$, (e) $t=42$ and (f) $t=62$. A periodic forcing $e^{i k_{o} x}, k_{o}=2$, has been applied at the upper boundary of the rectangular domain, $\delta=0.2$ and $\beta=1$. The background flow is constant, $\bar{u}=1$. . . .

5.7 Linear simulation of Rossby wave propagation $(\varepsilon=0)$ with the time-dependent radiation condition: contour plots of the streamfunction $\psi(x, y, t)$ obtained at (a) $t=2$, (b) $t=5$, (c) $t=10$, (d) $t=23$, (e) $t=42$ and (f) $t=62$. A periodic forcing $e^{i k_{o} x}$, $k_{o}=2$, has been applied at the upper boundary of the rectangular domain, $\delta=0.2$ and $\beta=1$. The background flow is constant, $\bar{u}=1.85$ 
5.8 Linear simulation of Rossby wave propagation: plot of the streamfunction $\psi(x, y, t)$ as a function of time $\mathrm{t}$ at $x=0.6$ and $y=-1$, $0 \leq t \leq 60$. A periodic forcing $e^{i k_{o} x}, k_{o}=2$, has been applied at the upper boundary of the rectangular domain, $\delta=0.2$ and $\beta=1$. The background flow is constant, $\bar{u}=1$. The solid line corresponds to the simulation with time-dependent radiation condition and the dashed-dotted line corresponds to the simulation with the zero boundary condition whereas the dashed line is the result obtained for the simulation with the steady radiation condition. .................... 86

5.9 Linear simulation of Rossby wave propagation: plot of the averaged horizontal momentum flux $F$ as a function of $y$ at the time $t=40$. A periodic forcing $e^{i k_{o} x}, k_{o}=2$, has been applied at the upper boundary of the rectangular domain, $\delta=0.2$ and $\beta=1$. The background flow is constant, $\bar{u}=1$. The solid line corresponds to the simulation with time-dependent radiation condition and the dashed-dotted line corresponds to the simulation with the zero boundary condition whereas the dashed line is the result obtained for the simulation with the steady radiation con-

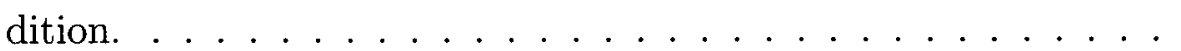


5.10 Linear simulation of Rossby wave propagation $(\varepsilon=0)$ with the time-dependent radiation condition in the long-wave limit $\delta=0$ : contour plots of the streamfunction $\psi(x, z, t)$ obtained at (a) $t=2$, (b) $t=5$, (c) $t=10$, (d) $t=23$, (e) $t=45$ and (f) $t=62$. A periodic forcing $e^{i k_{o} x}, k_{o}=2$, has been applied at the upper boundary of the rectangular domain and $\beta=1$. The background flow is constant, $\bar{u}=1 \ldots \ldots$. . . . . . . . . . . 91

5.11 Nonlinear simulation of Rossby wave propagation $(\varepsilon=0.05)$ with the zero boundary condition: contour plots of the streamfunction $\psi(x, z, t)$ obtained at (a) $t=10$, (b) $t=23$, (c) $t=42$, (d) $t=62$, (e) $t=80$ and (f) $t=85$. A periodic forcing $e^{i k_{o} x}, k_{o}=2$, has been applied at the upper boundary of the rectangular domain, $\delta=0.2$ and $\beta=1$. The background flow is constant, $\bar{u}=1 \ldots 92$

5.12 Nonlinear simulation of Rossby wave propagation $(\varepsilon=0.05)$ with the steady radiation condition, contour plots of the streamfunction $\psi(x, z, t)$ obtained at (a) $t=10$, (b) $t=23$, (c) $t=42$, (d) $t=62$, (e) $t=67$ and (f) $t=69$. A periodic forcing $e^{i k_{o} x}$, $k_{o}=2$, has been applied at the upper boundary of the rectangular domain, $\delta=0.2$ and $\beta=1$. The background flow is constant, $\bar{u}=1.93$

5.13 Nonlinear simulation of Rossby wave propagation $(\varepsilon=0.05)$ with the time-dependent radiation condition: contour plots of the streamfunction $\psi(x, z, t)$ obtained at (a) $t=10$, (b) $t=23$, (c) $t=42$, (d) $t=62$, (e) $t=80$ and (f) $t=85$. A periodic forcing $e^{i k_{o} x}$, $k_{o}=2$, has been applied at the upper boundary of the rectangular domain, $\delta=0.2$ and $\beta=1$. The background flow is constant, $\bar{u}=1.94$ 
5.14 Nonlinear simulation of Rossby wave propagation $(\varepsilon=0.05)$ : plot of the streamfunction $\psi(x, y, t)$ as a function of time $\mathrm{t}$ at $x=0.6$ and $y=-1,0 \leq t \leq 60$. A periodic forcing $e^{i k_{o} x}, k_{o}=2$, has been applied at the upper boundary of the rectangular domain, $\delta=0.2$ and $\beta=1$. The background flow is constant, $\bar{u}=1$. The solid line corresponds to the simulation with time-dependent radiation condition and the dashed-dotted line corresponds to the simulation with the zero boundary condition whereas the dashed line is the result obtained for the simulation with the steady radiation

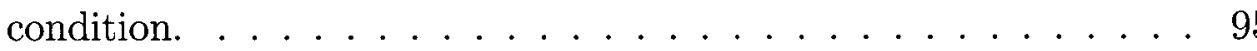

5.15 Nonlinear simulation of Rossby wave propagation $(\varepsilon=0.05)$ : contours plot of the streamfunction $\psi(x, y, t)$ at the time $t=5$ with, (a) the zero boundary condition, (b) the steady radiation condition , (c) the time-dependent radiation condition and (d) the zeros boundary condition on a large domain in the $y$-direction. A periodic forcing $e^{i k_{o} x}, k_{o}=2$, has been applied at the upper boundary of the rectangular domain and $\delta=0.2$. The background flow is constant, $\bar{u}=1 \ldots \ldots \ldots \ldots$. . . . . . . . . . 96 
5.16 Nonlinear simulation of Rossby wave propagation $(\varepsilon=0.05)$ : plot of the streamfunction $\psi(x=1.5, y=-1, t)$ as a function of time $t, 0<t<12$. A periodic forcing $e^{i k_{o} x}, k_{o}=2$, has been applied at the upper boundary of the rectangular domain and $\delta=0.2$. The background flow is constant, $\bar{u}=1$. The solid line corresponds to the zero boundary condition on a large domain in the $y$-direction, the dashed line corresponds to the time-dependent radiation condition, the dotted line corresponds to the steady radiation condition and the dashed-dotted line to the zero boundary

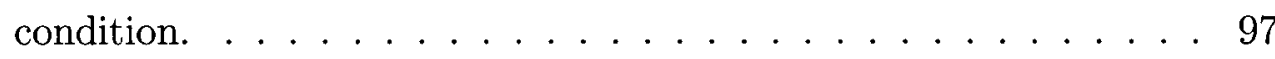

5.17 Nonlinear simulation of Rossby wave propagation $(\varepsilon=0.05)$ : plot of the averaged momentum flux $F(y=-1, t)$ as a function of time $t, 0<t<12$. A periodic forcing $e^{i k_{o} x}, k_{o}=2$, has been applied at the upper boundary of the rectangular domain and $\delta=0.2$. The background flow is constant, $\bar{u}=1$. The solid line corresponds to the zero boundary condition on a large domain in the $y$-direction, the dashed line corresponds to the time-dependent radiation condition, the dotted line corresponds to the steady radiation condition and the dashed-dotted line to the zero boundary

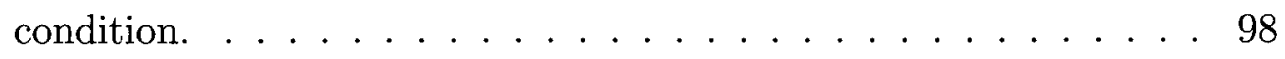


5.18 Nonlinear simulation of Rossby wave propagation $(\varepsilon=0.05)$ with the corrected time-dependent radiation condition: plot of $\hat{\psi}(\kappa, y, t)$ as a function of the wavenumber $\kappa$ at the time $t=125$ across the outflow boundary. A periodic forcing $e^{i k_{o} x}, k_{o}=2$, has been applied at the upper boundary of the rectangular domain, $\delta=0.2$ and $\beta=1$. The background flow is constant, $\bar{u}=1$. The solid line corresponds to the simulation with the time-dependent radiation condition without the nonlinear correction while the dashed line corresponds to the simulation with the time dependent radiation condition with the nonlinear correction. The difference between the two curve is barely noticeable at $(\kappa=0)$ since the zero wavenumber term and the nonlinear correction are very small compared with the magnitude of of $\hat{\psi}$ at the forcing $\left(\kappa= \pm k_{o}\right) \ldots 100$

5.19 Simulation of gravity wave propagation: the background flow speed is $\bar{u}=\tanh \left(z-z_{c}\right)$. The critical level is at $z_{c}=5 . \ldots 102$

5.20 Linear and nonlinear simulations of gravity wave propagation: contour plot of $\Psi(x, z, t)$ at $t=34,(a)$ linear simulation with $\epsilon=0$ and $(b)$ nonlinear simulation with $\epsilon=0.05$. In both $(a)$ and $(b) \delta=0.2$ and $R i=2$. A periodic forcing $e^{i k_{o} x}, k_{o}=2$, has been applied at the lower boundary of the rectangular domain. The background flow speed is $\bar{u}=\tanh \left(z-z_{c}\right)$, the critical level is at $z_{c}=5$. Both contour plots show that there is not much transmission of wave activity beyond the critical layer. . . . . . 104 
5.21 Linear and nonlinear simulations of gravity wave propagation: plot of the jump in the momentum flux $[F]$ across the critical layer as a function of time for $0 \leq t \leq 34$. A periodic forcing $e^{i k_{o} x}, k_{o}=2$, has been applied at the lower boundary of the rectangular domain, $\delta=0.2$ and $R i=2$. The background flow speed is $\bar{u}=\tanh \left(z-z_{c}\right)$, the critical level is at $z_{c}=5$. The dashed line shows the result obtained for the linear simulation $(\varepsilon=0)$ while the solid line shows the result obtained for the nonlinear simulation $(\varepsilon=0.05)$. In the linear case $[F]$ becomes almost constant by about $t=20$. This indicates absorption of the waves by the mean flow. In the nonlinear case $[F]$ alternates between the positive values (absorption) and the negative values (reflection). . 105

5.22 Linear simulation of gravity wave propagation $(\varepsilon=0)$ with the zero boundary condition: contour plots of the streamfunction $\psi(x, z, t)$ obtained at (a) $t=4$, (b) $t=8$, (c) $t=15$, (d) $t=35$, (e) $t=45$ and (f) $t=65$. A periodic forcing $e^{i k_{o} x}, k_{o}=2$, has been applied at the lower boundary of the rectangular domain and $\delta=0.2$. The background flow is constant, $\bar{u}=1 \ldots \ldots \ldots 8$

5.23 Linear simulation of gravity wave propagation $(\varepsilon=0)$ with the steady boundary condition: contour plots of the streamfunction $\psi(x, z, t)$ obtained at (a) $t=4$, (b) $t=8$, (c) $t=15$, (d) $t=35$, (e) $t=45$ and (f) $t=65$. A periodic forcing $e^{i k_{o} x}, k_{o}=2$, has been applied at the lower boundary of the rectangular domain and $\delta=0.2$. The background flow is constant, $\bar{u}=1 \ldots \ldots \ldots$ 
5.24 Linear simulation of gravity wave propagation $(\varepsilon=0)$ with the time-dependent radiation condition: contour plots of the streamfunction $\psi(x, z, t)$ obtained at (a) $t=4$, (b) $t=8$, (c) $t=15$, (d) $t=35$, (e) $t=45$ and (f) $t=65$. A periodic forcing $e^{i k_{o} x}$, $k_{o}=2$, has been applied at the lower boundary of the rectangular domain and $\delta=0.2$. The background flow is constant, $\bar{u}=1 \ldots 110$

5.25 Linear simulation of gravity wave propagation $(\varepsilon=0)$ : plot of the averaged vertical momentum flux $F$ as a function of $\mathrm{z}$ at the time $t=40$. A periodic forcing $e^{i k_{o} x}, k_{o}=2$, has been applied at the lower boundary of the rectangular domain and $\delta=0.2$. The background flow is constant, $\bar{u}=1$. The solid line corresponds to the simulation with the time-dependent radiation condition and the dotted line corresponds to the simulation with the zero boundary condition while the dashed line is the result obtained for the simulation with the steady radiation condition. . . . . 111

5.26 Linear simulation of gravity wave propagation $(\varepsilon=0)$ with the time-dependent radiation condition in the long-wave limit $\delta=0$ : contour plots of the streamfunction $\psi(x, z, t)$ obtained at (a) $t=4$, (b) $t=8$, (c) $t=15$, (d) $t=35$, (e) $t=45$ and (f) $t=65$. A periodic forcing $e^{i k_{o} x}, k_{o}=2$, has been applied at the lower boundary of the rectangular domain, $R i=2$. The background flow is constant $\bar{u}=1 \ldots \ldots \ldots 112$ 
5.27 Nonlinear simulation of gravity wave propagation $(\varepsilon=0.05)$ with the zero boundary condition: contour plots of the streamfunction $\psi(x, z, t)$ obtained at (a) $t=4$, (b) $t=8$, (c) $t=15$, (d) $t=35$, (e) $t=45$ and (f) $t=62$. A periodic forcing $e^{i k_{o} x}, k_{o}=2$, has been applied at the lower boundary of the rectangular domain and $\delta=0.2$. The background flow is constant, $\bar{u}=1 \ldots \ldots \ldots 115$

5.28 Nonlinear simulation of gravity wave propagation $(\varepsilon=0.05)$ with the steady radiation condition: contour plots of the streamfunction $\psi(x, z, t)$ obtained at (a) $t=4$, (b) $t=8$, (c) $t=15$, (d) $t=35$, (e) $t=45$ and (f) $t=60$. A periodic forcing $e^{i k_{o} x}, k_{o}=2$, has been applied at the lower boundary of the rectangular domain and $\delta=0.2$. The background flow is constant, $\bar{u}=1 \ldots \ldots 116$

5.29 Nonlinear simulation of gravity wave propagation $(\varepsilon=0.05)$ with the time-dependent radiation condition: contour plots of the streamfunction $\psi(x, z, t)$ obtained at (a) $t=4$, (b) $t=8$, (c) $t=15$, (d) $t=35$, (e) $t=45$ and (f) $t=62$. A periodic forcing $e^{i k_{o} x}$, $k_{o}=2$, has been applied at the lower boundary of the rectangular domain and $\delta=0.2$. The background flow is constant, $\bar{u}=1 \ldots 117$

5.30 Nonlinear simulation of gravity wave propagation $(\varepsilon=0.05)$ : contours plot of the streamfunction $\psi(x, z, t)$ at time $t=8$ with, (a) the zero boundary condition, (b) the steady radiation condition , (c) the time-dependent radiation condition and (d) the zeros boundary condition on a large domain. A periodic forcing $e^{i k_{o} x}$, $k_{o}=2$, has been applied at the lower boundary of the rectangular domain and $\delta=0.2$. The background flow is constant, $\bar{u}=1 \ldots 118$ 
5.31 Nonlinear simulation of gravity wave propagation $(\varepsilon=0.05)$ : plot of the streamfunction $\psi(x=1.5, z=6, t)$ as a function of time $t$, $0<t<10$. A periodic forcing $e^{i k_{o} x}, k_{o}=2$, has been applied at the lower boundary of the rectangular domain and $\delta=0.2$. The background flow is constant, $\bar{u}=1$. The solid line corresponds to the zero boundary condition on a large domain in the $z$-direction, the dashed line corresponds to the time-dependent radiation condition, the dashed-dotted line corresponds to the steady radiation condition and the dotted line to the zero boundary condition. . . 119

5.32 Nonlinear simulation of gravity wave propagation $(\varepsilon=0.05)$ : plot of the averaged momentum flux $F(z=6, t)$ as a function of time $t, 0<t<10$. A periodic forcing $e^{i k_{o} x}, k_{o}=2$, has been applied at the lower boundary of the rectangular domain and $\delta=0.2$. The background flow is constant, $\bar{u}=1$. The solid line corresponds to the zero boundary condition on a large domain in the $z$-direction, the dashed line corresponds to the time-dependent radiation condition, the dashed-dotted line corresponds to the steady radiation condition and the dotted line to the zero boundary condition. . . 120 
5.33 Nonlinear simulation of gravity wave propagation $(\varepsilon=0.05)$ : plot of $\hat{\psi}(\kappa, z, t)$ as a function of the wavenumbers $\kappa$ across the outflow boundary at the time $t=65$. A periodic forcing $e^{i k_{o} x}, k_{o}=2$, has been applied at the lower boundary of the rectangular domain and $\delta=0.2$. The background flow is constant, $\bar{u}=1$. The dashed line corresponds to the time-dependent radiation condition with the the nonlinear correction (3.76) while the solid line corresponds to the time-dependent radiation condition without the nonlinear correction. . . . . . . . . . . . . . . . . . . 122

5.34 Nonlinear simulation of gravity wave propagation $(\varepsilon=0.05)$ : plot of wave-induced mean streamfunction $\bar{\psi}_{0}(z, t)$ as a function of $z$ at the time $t=65$. The dashed line corresponds to the timedependent radiation condition with the nonlinear correction while the solid line corresponds to the time-dependent radiation condition without the correction. A periodic forcing $e^{i k_{o} x}, k_{o}=2$, has been applied at the lower boundary of the rectangular domain and $\delta=0.2$. The background flow is constant, $\bar{u}=1 \ldots \ldots \ldots 123$ 


\section{List of Symbols}

- $x$ horizontal or zonal space variable

- $y$ meridional space variable or latitude

- $z$ vertical space variable or altitude

- $t$ time variable

- $\hat{x}$ unit vector in the $x$-direction

- $\hat{y}$ unit vector in the $y$-direction

- $\hat{z}$ unit vector in the $z$-direction

- $\Psi$ total streamfunction

- $\psi$ perturbation of streamfunction

- $\bar{\psi}$ mean of the streamfunction

- $\bar{\psi}_{0}$ wave-induced mean streamfunction

- $\xi$ total vorticity

- $\bar{\zeta}$ mean of the total vorticity

- $\zeta$ perturbation of vorticity

- $\varrho$ total stratified density

- $\varrho_{o}$ constant reference density

- $\rho$ perturbation density 
- $\bar{\rho}$ mean density or hydrostatic density

- $\rho_{o}$ constant reference density density

- $\varphi$ amplitude of the streamfunction at the source

- $\phi$ amplitude of the streamfunction near the outflow boundary

- $k_{o}$ wavenumber at the forcing

- $\kappa$ wavenumber in the $x$-direction

- $\vec{u}$ velocity of the fluid

- $u x$-component of the velocity of the fluid

- $\bar{u}$ mean flow velocity

- $\bar{u}_{0}$ wave-induced mean velocity

- $v y$-component of the velocity of the fluid

- $w z$-component of the velocity of the fluid

- $\Lambda$ wavevector

- $k x$-component of the wavevector

- $l y$-component of the wavevector

- $m z$-component of the wavevector

- $\beta$ gradient of the planetary vorticity in the $y$-direction

- $B$ typical scale of the gradient of the planetary vorticity in the $y$ direction 
- $f$ Coriolis factor

- $\vec{\Omega}$ angular velocity of the earth rotation

- $\theta$ latitude angle

- $H_{o}$ scale height of the atmosphere

- $N^{2}$ square of Brunt Väisälä frequency

- $R i$ Richardson number

- $L_{x}$ typical length scale in the horizontal or zonal direction

- $L_{y}$ typical length scale in the meridional direction

- $L_{z}$ typical length scale in the vertical direction

- $M$ typical scale for the mass

- $U$ typical scale for the velocity

- $V$ typical scale for the volume

- $p$ pressure of the fluid

- $\bar{p}$ hydrostatic pressure

- $T$ temperature of the fluid

- $\delta$ square root of the aspect ratio

- $\varepsilon$ characteristic parameter of the nonlinearity of the governing equations

- $\vec{g}$ acceleration due to gravity 
- $\vec{\eta}$ exterior force or superposition of exterior forces

- $\eta$ magnitude of the exterior force

- $\eta_{1} x$-component of the exterior force

- $\eta_{2} y$-component of the exterior force

- $\eta_{3} z$-component of the exterior force

- $\vec{F}_{c}$ centripetal force

- $F$ momentum flux

- $[F]$ jump in the momentum flux

- $R$ Radius of the earth

- $\omega$ pulsation or intrinsic frequency

- $\vec{C}$ phase velocity

- $c=C_{x}$ phase velocity in the $x$-direction

- $C_{y}$ phase velocity in the $y$-direction

- $C_{z}$ phase velocity in the $z$-direction

- $\vec{C}_{g}$ group velocity

- $C_{g x}$ group velocity in the $x$-direction

- $C_{g y}$ group velocity in the $y$-direction

- $C_{g z}$ group velocity in the $z$-direction 
- $C_{p}$ heat capacity at constant pressure

- $C_{V}$ heat capacity at constant volume

- $e$ internal energy

- $h$ enthalpy

- $q$ thermal energy (heat)

- $\vec{q}$ heat flux vector per unit of area

- $\emptyset$ viscous dissipation

- $\mu$ constant of viscosity

- $\nu$ kinematic viscosity

- $\mathcal{V}$ typical scale for the kinematic viscosity

- $\mathcal{R}$ constant for a perfect gas

- $\mathcal{K}$ thermal conductivity

- $\alpha$ thermal diffusivity

- $\mathcal{A}$ typical scale for the thermal diffusivity

- $\kappa$ Fourier transform variable in the $x$-direction

- $s$ Laplace transform variable

- $J(\psi, \zeta)$ Jacobian of $\psi$ and $\zeta$

- $J(\psi, \rho)$ Jacobian of $\psi$ and $\rho$ 
- $\hat{\psi}$ Fourier transform of $\psi$

- $\tilde{\psi}$ Fourier-Laplace transform of $\psi$

- $\hat{\xi}$ Fourier transform of $\xi$

- $\hat{\zeta}$ Fourier transform of $\zeta$

- $\hat{\rho}$ Fourier transform of $\rho$

- $\hat{\phi}$ Fourier transform of $\phi$

- $\mathcal{F}$ Fourier transform

- $\mathcal{F}^{-1}$ Inverse Fourier transform

- DFT discrete Fourier transform

- FFT fast Fourier transform

- $\hat{J}(\psi, \zeta) D F T$ of the Jacobian of $\psi$ and $\zeta$

- $\hat{J}(\psi, \rho) D F T$ of the Jacobian of $\psi$ and $\rho$

- IDFT inverse discrete Fourier transform

- IFFT inverse fast Fourier transform

- $\mathcal{L}$ Laplace transform

- $\mathcal{L}^{-1}$ inverse Laplace transform

- $\Delta x, \Delta y, \Delta z, \Delta t$ increments in $x, y, z$ and $t$ 


\section{Chapter 1}

\section{Introduction}

\subsection{Wave simulations in geophysical fluids}

Waves in fluids can be simulated with mathematical models based on the equations for conservation of mass, momentum and energy, with the waves being represented as perturbations to the steady basic flow. In geophysical fluid dynamics, the effects of the earth's rotation and the density-stratification of the basic flow are taken into account. There are various types of waves in geophysical flows in the atmosphere and ocean. These include internal gravity waves which result from the effects of gravity and the buoyancy of the fluid flow, and Rossby waves which result from the effects of the earth's rotation. In this thesis we will focus on these two types of waves.

Internal gravity waves propagate along the orthogonal axis of the earth's tangent plane, the $z$-axis (Nappo, 2002). Due to changes in the fluid density in the atmosphere (stratified density), there is variation in the buoyancy forces. The equilibrium is restored by generating the gravity waves. These waves transport momentum and energy within them and they produce large scale effects on the 
general circulation of the atmosphere and ocean. Stratospheric sudden warming and clear air turbulence are some of the observed phenomena that result from gravity waves interactions. Thus, it is important for weather prediction and climate modeling to characterize the properties of these waves. Since gravity waves are relatively small-scale waves with wavelength of about $1-100 \mathrm{~km}$, they are not generally resolved by large scale general circulation models. The effects of gravity waves are included in these models by means of parametrization which are based on the mathematical theory of gravity waves.

A simple two-dimensional mathematical model for gravity waves consists of a rectangular domain in a plane perpendicular to the surface of the earth with Cartesian coordinates, $x$ in the horizontal direction and $z$ in the vertical direction. The gravity waves are considered to be perturbations to some basic background flow. The background flow velocity has no vertical component and its horizontal component is the horizontal mean flow speed, for example it could be represented as

$$
\vec{u}=(\bar{u}(z), 0)
$$

This configuration allows mathematical analyses using perturbation theory and both linear and nonlinear numerical simulations. It is assumed that the waves are generated at one boundary of the domain of interest by a source such as convection or storms. Gravity waves generated in the lower atmosphere generally propagate upwards into the stratosphere. So the source level of the waves can be considered to be the lower boundary of the rectangular domain and in theory the upper boundary should be at infinity, in other word the domain should be unbounded above in the $z$-direction.

On the other hand, Rossby (or planetary) waves are giant meanders in high- 
altitude winds that are a major influence of the weather (Holton, 2000). They result from the rotation of the earth, specially the variation of the Coriolis effect with latitude. Cyclones and anticyclones are typical phenomena which are induced by Rossby waves interactions in the atmosphere.

From a mathematical perspective, Rossby waves can be studied either in 3 dimensions using spherical coordinates or in 2 dimensions using rectangular coordinates in a plane tangent to the surface of the earth, $x$ for the west-east (zonal) coordinate and $y$ for the south-north (meridional) coordinate. The background flow velocity in this case has no meridional component and its zonal component is is the zonal mean flow, ie.

$$
\vec{u}=(\bar{u}(y), 0)
$$

Rossby waves, in the northern hemisphere, are frequently generated at midlatitudes and propagate southwards towards the equator. In a two dimensional model in rectangular geometry it is assumed that the waves are forced at the northern boundary of the rectangular domain. In theory, the domain should be assumed to be infinite allowing the waves to propagate indefinitely southwards.

In practice in numerical simulation, we must limit the waves to a finite domain, this means that once the waves get to the opposite boundary, they are prevented from propagating further and often there are instabilities which may change the physical nature or behavior of the disturbance. In other to avoid such instabilities, we need to apply appropriate boundary conditions at the outflow boundary. These are known as non-reflecting boundary conditions or radiation conditions. Ideally, non-reflecting boundary conditions should be nonlinear and time-dependent, but in practice they are generally based on linearized steady equations. This thesis examines time-dependent radiation conditions for mod- 
eling the propagation of atmospheric gravity waves and Rossby waves in both linear and nonlinear configurations.

\subsection{Previous work}

In the past, different techniques have been tried by different researchers in order to simulate the behavior of fluids on an infinite domain using a finite computational domain. A frequently used method developed in the past 40 years consists of surrounding the outflow boundary of the computational boundary with a sponge layer, which is a layer of high viscosity, in order to dissipate the energy of incident waves near the interested boundary. The sponge layer technique is commonly used in large scale numerical models, e.g. general circulation models

of the atmosphere. The viscosity in the sponge layer is represented using either Rayleigh friction of the form

$$
\frac{\partial \psi}{\partial t}+\ldots . .=-\gamma(y) \psi
$$

or second-order viscosity of the form

$$
\frac{\partial \psi}{\partial t}+\ldots \ldots=\gamma(y) \nabla^{2} \psi
$$

where $\psi$ is the wave variable and $\gamma$ is a function of the physical space variable $y$ that is large near the outflow and close to zero elsewhere. However in this method the addition of the viscosity is artificial and it changes the physical nature of the waves at the boundary. The viscosity is generally added only in the sponge layer and that makes the physical nature of the waves in the sponge 
layer different from the rest of the domain.

Another approach is to modify the flow characteristics at large distances from the wave source transforming the region near the outflow boundary into an evanescent region and thereby absorbing the waves and preventing reflection near the outflow boundary. This technique removes the waves from the computational domain so that there is no propagation. This idea was used by Geisler and Dickinson (1974) for Rossby waves propagation and is an example of the buffer zone technique which is used in a wide variety of different contexts in fluid dynamics.

Another idea was described by Pearson (1974); he suggested fixing the wavelength by the means of the group velocity based on the time that it takes the wave to propagate up to the outflow boundary and assuming the wave amplitude is constant at the outflow boundary

$$
\psi \sim e^{ \pm i l(y-c t)}
$$

In the context of geophysical fluid simulations this was the starting point of developing what we call today radiation conditions. At the outflow boundary we impose a boundary condition of the form

$$
\frac{\partial \psi}{\partial y}= \pm i l \psi
$$

which implies that the wave amplitude is of the form (1.5) and one can choose the sign that corresponds to a solution with outward energy propagation. However, this type of radiation condition does not take into account the transient evolution of waves. Béland and Warn (1975) developed a time-dependent radiation 
condition for Rossby waves; their radiation condition is derived by linearizing the governing equations and making use of a Laplace transform. One drawback of their method is that the boundary condition is in terms of a Laplace convolution integral in time, so it is non-local in time and expensive to compute, requiring values of the dependent variable at all previous time levels. Also, when applied to a nonlinear problem instabilities sometimes develop at late time. Béland and Warn (1975) noted that the rate of degradation of their simulations depended on both the intensity of the nonlinearities and the scale of motion near the computational boundary.

A time-dependent radiation condition for gravity waves also based on Laplace transforms was first derived by Campbell and Maslowe (2003). This radiation condition suffers from the same drawbacks, it is expensive to evaluate the Laplace convolution integral and it does not always work for the nonlinear problem. Campbell (2004) and Campbell and Maslowe (2003) used these linear radiation conditions for their simulations of Rossby and gravity waves respectively. Both of these studies dealt with configurations where there is a critical line in the flow. A critical line or a critical level is a position in space where the velocity of the wave is equal to speed of the shear flow and the adjacent region to the critical level is called the critical layer. The waves are absorbed or reflected at the critical level and do not reach the outflow boundary. In both studies they concluded that it was enough to simply apply a zero boundary condition at the outflow. However, in a configuration where there is no critical level the waves do reach the outflow boundary and it is necessary to correctly implement the radiation condition. Ideally the radiation condition should take into account nonlinearity as well and time dependence. 
In this thesis techniques are developed to approximate the convolution integral so as to make the computations local in time and hence less expensive. Numerical simulations are then carried out using these time-dependent linear radiation conditions and the results are compared with the results obtained by simply setting the fluid variables to zero at the outflow boundary. The results are assessed using our knowledge of the behavior of the exact solution. We observe that, with zero boundary conditions, inaccurate results are obtained and instabilities may develop, while the simulations with the radiation conditions are able to maintain their stability for a prolonged period of time. However, if the simulations are continued to even later times, the linear radiation conditions inevitably fail when the nonlinear terms become large near the outflow boundary. The ultimate goal would be to develop a fully nonlinear radiation condition.

In this thesis we will include the effects of nonlinearity in the time-dependent radiation condition by adding a nonlinear correction which is based on weakly nonlinear perturbation theory and we find that this improves the results of the nonlinear simulations.

\subsection{An overview of the thesis}

An overview of the thesis is as follows:

In Chapter 2, we derive and discuss the governing equations for the twodimensional propagation of both Rossby waves and gravity waves starting with the basic equations of fluid dynamics that describe conservation of mass, momentum and energy. In both cases, we consider the background fluid to be a shear flow, i.e. for Rossby waves the background flow has only a zonal velocity component $\bar{u}(y)$ that varies with latitude $y$ with no meridional component, 
and for gravity waves the background flow has density $\bar{\rho}(z)$ and a horizontal velocity component $\bar{u}(z)$ both of which vary with height and there is no vertical velocity component. We present the beta-plane approximation for Rossby wave propagation and the Boussinesq approximation for gravity waves.

In Chapter 3, we derive radiation conditions for both Rossby and gravity waves. We first derive the steady radiation condition or the steady non-reflecting boundary condition by assuming the amplitude of the waves does not depend on time. Next, we derive the time-dependent linear non-reflecting boundary condition for which the amplitude of the waves is assumed to depend on time. These are the boundary conditions developed by Béland and Warn (1975) and by Campbell and Maslowe (2003) for Rossby and gravity waves respectively. In order to derive the time-dependent radiation condition we take a Laplace transform in time, this leads to a Laplace convolution integral which is nonlocal in time. Finally we describe how to add a nonlinear correction to the timedependent linear radiation condition. To do this we make use of weakly-nonlinear theory, i.e. we seek a solution of the form $\psi=\psi^{(0)}+\varepsilon \psi^{(1)}$ where $\psi^{(1)}$ is obtained from the substitution of $\psi$ into the nonlinear equation while $\psi^{(0)}$ is the solution obtained if a linear radiation condition is used.

In Chapter 4, we discuss about the numerical methods, finite difference and pseudospectral approximations, that are used in this study. We use the discrete Fourier transform $(D F T)$ to descretize all the quantities with respect to the $x$ variable, this allows us to apply the pseudospectral approximation to the nonlinear terms (Jacobian) and thus utilize the fast Fourier transform algorithm $(F F T)$. Central finite difference approximations are used in the $y$ and $z$ directions inside the rectangular domain for Rossby and gravity waves respectively, 
the backward finite difference approximation is used at the outflow boundary while the forward finite difference approximation is used for the time discretization. In this thesis we describe an appropriate way to implement the radiation condition to make the implementation of the Laplace convolution integral local in time.

In Chapter 5 we present the results of the simulations. The first set of results are for the case where the mean flow profile is a hyperbolic tangent profile. So there is a critical layer in the flow. This is the configuration studied by Campbell and Maslowe (2003) and Campbell (2004). With this mean flow profile there is a critical level in the flow and the waves are absorbed or reflected in the critical layer and we don't need the radiation condition. Our simulations however focus on configurations in which there is no critical layer and so waves propagate all the way to the outflow boundary where a non-reflecting radiation is applied. We present the results for gravity wave and Rossby wave propagations from both linear and nonlinear simulations.

The new and original ideas presented in this thesis are the following:

- The derivation of an exact solution for a transient forced Rossby wave in the form of an infinite series (section 2.5 and Appendix D) using Laplace transform.

- The extension of the linear time-dependent radiation condition to include a nonlinear correction (section 3 ).

- The numerical implementation of the time-dependent radiation condition making the computation of the Laplace convolution integral local in time (Chap 3). 
- The development of MATLAB programs to solve the linear and nonlinear Rossby wave and gravity wave problems using spectral and finite difference method (Chap 4).

- A comparison of the results obtained with different outflow boundary conditions. 


\section{Chapter 2}

\section{Governing equations}

\subsection{The basic equations of fluid dynamics}

In this section we present the basic equations of fluid dynamics which are based on the physical principles of conservation of mass, momentum and energy. These equations are the starting point for modeling the propagation of Rossby waves and gravity waves in the atmosphere. The derivation of the basic equations can be found in any fluid dynamics text such as Batchelor (1967) and Kundu and Cohen (2004).

We will make the Boussinesq approximation which is a commonly used approximation for studying geophysical flows. It is described in details by Spiegel and Veronis (1960). Under the Boussinesq approximation the fluid density and temperature are treated as constant except in the terms involving the effects of gravity in the governing equations. The assumptions made in the Boussinesq 
approximation are:

1. The variation of the density $\varrho$ in time and space is small enough that we can set $\frac{1}{\varrho} \frac{D \varrho}{D t} \approx 0$, where $\frac{D}{D t}$ denotes the rate of change of $\varrho$ following the flow.

2. We also assume $\frac{\partial \varrho}{\partial p} \approx 0$, i.e. the fluid is almost incompressible or the density does not change much with the pressure $p$.

3. The vertical scale height is small enough that the density varies by only a small amount in the vertical direction.

4. The fluid properties such as $\mu$ the viscosity coefficient and $\mathcal{K}$ the thermal conductivity are assumed to be constant and the viscous dissipation can be neglected.

\subsubsection{The Navier-Stokes equations}

The principle of conservation of momentum (Newton's second law) applied to incopressible fluid flows leads to the Navier-Stokes equation which is given by

$$
\varrho \frac{D \vec{u}}{D t}=-\nabla p+\varrho \vec{g}+\mu \nabla^{2} \vec{u}+\vec{\eta}
$$

where $\vec{\eta}$ is an exterior force or a superposition of two or more exterior forces that could include the Coriolis force, the centrifugal or centripetal force, electric or magnetic force, depending on the problem that one needs to model. $\varrho$ is the density of the fluid, $\vec{u}$ is the velocity of the fluid motion and in rectangular coordinates has 3 components $u, v$ and $w$, respectively in $x, y$ and $z$-directions, $\vec{g}=(0,0,-g)$ is the acceleration due to gravity and the differential operator $\frac{D}{D t}$ 
is given by

$$
\frac{D}{D t}=\frac{\partial}{\partial t}+u \frac{\partial}{\partial x}+v \frac{\partial}{\partial y}+w \frac{\partial}{\partial z}
$$

The term $\mu \nabla^{2} \vec{u}$ represents the effects of viscosity or friction in the fluid, $\mu$ is the viscosity coefficient and $\varrho$ and $p$ are the density and the pressure of the fluid respectively. In Cartesian coordinates, equation (2.1) is written as

$$
\begin{aligned}
& \varrho \frac{D u}{D t}=-\frac{\partial p}{\partial x}+\mu \nabla^{2} u+\eta_{1} \\
& \varrho \frac{D v}{D t}=-\frac{\partial p}{\partial y}+\mu \nabla^{2} v+\eta_{2} \\
& \varrho \frac{D w}{D t}=-\frac{\partial p}{\partial z}-\varrho g+\mu \nabla^{2} w+\eta_{3}
\end{aligned}
$$

For Rossby waves the vector $\vec{\eta}$ represents the sum of the Coriolis force and the centripetal force and is given by

$$
\vec{\eta}=(\varrho f v,-\varrho f u, 0)+\vec{F}_{c}
$$

where $f$ is the Coriolis parameter. The centripetal force $\vec{F}_{c}$ is orthogonal to the direction of propagation of the Rossby waves so its net contribution is zero. So $\vec{\eta}$ is just the Coriolis force and is given by

$$
\vec{\eta}=(\varrho f v,-\varrho f u, 0)
$$

In our 2-D model for Rossby waves, the fluid undergoes rotations in the $x y$ plane. So the force due to gravity is orthogonal to the propagation of the waves and its effects are not included in the model. The Rossby wave model comprises equations (2.3) and (2.4) for the horizontal velocity components $u$ and $v$. 
Gravity waves on the other hand propagate in a direction that is approximately perpendicular to the earth's tangent plane, so the effect of the Coriolis force is negligible and we can neglect the $y$-component because the information contained in $y$-component is also in the $x$-component. The effect of the force due to gravity on the other hand is more important. The variation of the density is compensated by the restoring force (buoyancy force), consequently there is an important change in the force due to gravity. Therefore the gravity wave model comprises only equations (2.3) and (2.5) for the horizontal velocity component $u$ and the vertical velocity component $w$.

\subsubsection{The continuity equation}

The principle of conservation of mass of a fluid leads to the continuity equation which is given in rectangular coordinates by

$$
\frac{1}{\varrho} \frac{D \varrho}{D t}+\left[\frac{\partial u}{\partial x}+\frac{\partial v}{\partial y}+\frac{\partial w}{\partial z}\right]=0
$$

In conditions where $\frac{D \varrho}{D t}$ is small relative to the density $\varrho$ (for example under the assumptions of the Boussinesq approximation) we can say that $\frac{D Q}{D t} \approx 0$ and the continuity equation can be written as

$$
\frac{\partial u}{\partial x}+\frac{\partial v}{\partial y}+\frac{\partial w}{\partial z}=0
$$

Thus in a horizontal plane the continuity equation is given by

$$
\frac{\partial u}{\partial x}+\frac{\partial v}{\partial y}=0
$$


while in a vertical plane the continuity equation is given by

$$
\frac{\partial u}{\partial x}+\frac{\partial w}{\partial z}=0
$$

\subsubsection{The energy equation}

In our gravity wave model, the conservation of energy is given by

$$
\varrho \frac{D e}{D t}=-\nabla \cdot \vec{q}-p \nabla \cdot \vec{u}+\varnothing
$$

where $e$ represents the internal energy, $\varnothing$ is the viscous dissipation and $\vec{q}$ is the heat flux vector (per unit of area). The equation of state, assuming the energy is a function of temperature $T$ only, is given by

$$
e(T)=C_{V} T
$$

where $C_{v}$ is the specific heat capacity at constant volume $V$ and given by

$$
C_{V}=\left(\frac{\partial e}{\partial T}\right)_{V}
$$

where the subscript $V$ indicates that the differentiation with respect to $T$ is carried out with fixed $V$. The relation between the pressure $p$, the density $\varrho$ and the temperature $T$ is given by the equation of state for a perfect gas,

$$
p=\mathcal{R} \varrho T
$$


where $\mathcal{R}=C_{p}-C_{v}$ is a constant for a given perfect gas. $C_{p}$ is the specific heat capacity at constant pressure $p$ and is given by

$$
C_{p}=\left(\frac{\partial h}{\partial T}\right)_{p}
$$

where $h$ is the enthalpy. Under the Boussinesq approximation we assume $\nabla \cdot \vec{u}=0$ in the continuity equation because $\frac{1}{\varrho} \frac{D \varrho}{D t}$ is small. However we write the continuity equation as $\nabla \cdot \vec{u}=0$ we can not justify neglecting $p \nabla \cdot \vec{u}$ term in (2.12) because although the $\nabla \cdot \vec{u}$ is small the pressure can be large and the product $p \nabla \cdot \vec{u}$ can be of the same order of magnitude as other terms in (2.12). So we keep $p \nabla \cdot \vec{u}$ term and rewrite it in terms of $\varrho$ and $T$.

According to the continuity equation (2.8) can be written as

$$
\frac{1}{\varrho} \frac{D \varrho}{D t}=-\nabla \cdot \vec{u}
$$

which implies that

$$
-p \nabla \cdot \vec{u}=\frac{p}{\varrho} \frac{D \varrho}{D t}=\frac{p}{\varrho}\left[\left(\frac{\partial \varrho}{\partial T}\right) \frac{D T}{D t}+\left(\frac{\partial \varrho}{\partial p}\right) \frac{D p}{D t}\right]
$$

assuming the density depends on both temperature and pressure. The second term on the right hand side is small relative to the first term according to assumptions 2 of the Boussinesq approximation given on page 12. So it can be neglected to obtain

$$
-p \nabla \cdot \vec{u}=\frac{p}{\varrho} \frac{D \varrho}{D t} \approx \frac{p}{\varrho}\left(\frac{\partial \varrho}{\partial T}\right)_{p} \frac{D T}{D t}
$$

where the subscript $p$ denotes differentiation of $\varrho$ with respect to $T$ with the 
pressure being kept fixed.

Using the perfect gas law (2.15), equation (2.19) becomes,

$$
\begin{array}{r}
-p \nabla \cdot \vec{u}=\frac{p}{\varrho} \frac{D}{D t}\left(\frac{p}{\mathcal{R} T}\right) \approx \frac{p}{\varrho}\left(\frac{\partial}{\partial T}\left(\frac{p}{\mathcal{R} T}\right)\right)_{p} \frac{D T}{D t} \\
=\frac{p}{\varrho} \frac{p}{\mathcal{R}}\left(-\frac{1}{T^{2}}\right) \frac{D T}{D t}=-\mathcal{R} \varrho \frac{D T}{D t}
\end{array}
$$

Combining this equation with the energy equation (2.12) yields

$$
\varrho \frac{D e}{D t}=-\nabla \cdot \vec{q}-\mathcal{R} \varrho \frac{D T}{D t}+\varnothing
$$

If we combine equation (2.21) with equation (2.13), use the fact that $\mathcal{R}=C_{p}-C_{V}$ and rearrange the terms, we obtain

$$
C_{p} \varrho \frac{D T}{D t}=-\nabla \cdot \vec{q}+\varnothing
$$

According to Fourier's law of heat conduction $\vec{q}$ is given by

$$
\vec{q}=-\mathcal{K} \nabla T
$$

where $\mathcal{K}$ is the thermal conductivity.

For geophysical flows under the Boussinesq approximation the ratio of the magnitude of viscous dissipation $\varnothing$ to the term $C_{p} \varrho \frac{D T}{D t}$ is extremely small ( $10^{-7}$ ). This can be shown using dimensional analysis (Kundu and Cohen 2004. p 120). We thus are justified in neglecting the viscous dissipation. We then 
substitute (2.23) into equation (2.22) to get

$$
C_{p} \varrho \frac{D T}{D t}=-\nabla \cdot(-\mathcal{K} \nabla T)=\mathcal{K} \nabla^{2} T
$$

Rearranging the terms yields a single equation for the temperature

$$
\frac{D T}{D t}=\alpha \nabla^{2} T
$$

where $\alpha$, the thermal diffusivity, is given by

$$
\alpha=\frac{\mathcal{K}}{C_{p} \varrho}
$$

In section (2.3), we will use equation (2.25) to derive the equation that describes the density stratification in the atmosphere .

\subsection{The governing equations for Rossby waves}

In this section, we derive an equation known as the barotropic vorticity equation, to model Rossby waves in a horizontal plane, starting from the momentum equations (2.3), (2.4) and the continuity equation in the horizontal plane (2.10). We consider Rossby waves propagating in a horizontal plane and thus neglect variations of fluid properties in the vertical direction. A two dimensional fluid with no vertical variations is an example of a barotropic fluid, i.e., a fluid for which the pressure is a function of density only, and vice versa. So surfaces of constant pressure are the same as surfaces of constant density. Thus the momentum equations we use to model the propagation of Rossby wave in the 
atmosphere are,

$$
\begin{aligned}
& \frac{\partial u}{\partial t}+u \frac{\partial u}{\partial x}+v \frac{\partial u}{\partial y}=-\frac{1}{\varrho} \frac{\partial p}{\partial x}+\nu \nabla^{2} u+f v \\
& \frac{\partial v}{\partial t}+u \frac{\partial v}{\partial x}+v \frac{\partial v}{\partial y}=-\frac{1}{\varrho} \frac{\partial p}{\partial y}+\nu \nabla^{2} w-f u
\end{aligned}
$$

where $\nu=\frac{\mu}{\varrho}$ is the kinematic viscosity and $f$ is the Coriolis factor.

\subsubsection{The beta plane approximation and the Coriolis force}

For a thin layer of fluid of constant density on a rotating sphere, the Coriolis force $\vec{\eta}$ is given (see for example Kundu and Cohen 2004) by

$$
\vec{\eta}=(2 \Omega v \sin \theta,-2 \Omega u \sin \theta)=(f v,-2 f u)
$$

where

$$
f=2 \Omega \sin \theta
$$

is called the Coriolis factor, $\Omega$ the magnitude of the angular velocity of the earth rotation and $\theta$ the latitude. In the beta-plane approximation we consider some fixed latitude $\theta_{o}$ where the Coriolis factor is $f_{o}=2 \Omega \sin \theta_{o}$ and then assume that $\theta$ is close enough to $\theta_{o}$ that we can expand $f$ as a Taylor series about the latitude $\theta=\theta_{o}$ and approximate it by the first two terms. We also use the approximation for the arclength $y \approx R\left(\theta-\theta_{o}\right)$ and therefore the Coriolis factor can be approximated as 


$$
f \approx f_{o}+\left(\frac{d f}{d \theta}\right)_{\theta=\theta_{o}}\left(\theta-\theta_{o}\right) \approx f_{o}+\left(\frac{d f}{d y}\right)_{y=0} \frac{y}{R}=f_{o}+\beta y
$$

where $\beta=\left(\frac{\partial f}{\partial y}\right)_{y=0}=\frac{1}{R}\left(\frac{d f}{d \theta}\right)_{\theta=\theta_{o}}=\frac{2 \Omega}{R} \cos \theta_{o}$ is the gradient of planetary vorticity in $y$-direction (see for example Kundu and Cohen, 2004).

\subsubsection{The barotropic vorticity equation}

In order to derive the barotropic vorticity equation (Holton, 1975), we first differentiate equation (2.27) with respect to $y$, then equation (2.28) with respect to $x$ and subtract the first equation from the second to get

$$
\begin{array}{r}
\left(v_{x}-u_{y}\right)_{t}+u_{x}\left(v_{x}-u_{y}\right)+u\left(v_{x}-u_{y}\right)_{x}+v_{y}\left(v_{x}-u_{y}\right)+v\left(v_{x}-u_{y}\right)_{y} \\
=\nu \nabla^{2}\left(v_{x}-u_{y}\right)+\beta v
\end{array}
$$

where the subscripts $x, y$ and $t$ denote the partial differentiation with respect to $x, y$ and $t$ respectively. Substituting $u_{x}=-v_{y}$ from the the continuity equation(2.10) and rearranging the terms yields

$$
\left(v_{x}-u_{y}\right)_{t}+u\left(v_{x}-u_{y}\right)_{x}+v\left(v_{x}-u_{y}\right)_{y}=\nu \nabla^{2}\left(v_{x}-u_{y}\right)+\beta v
$$

The continuity equation (2.10) allows us to define a streamfunction $\Psi$ by $v=\frac{\partial \Psi}{\partial x}$ and $-u=\frac{\partial \Psi}{\partial y}$. This implies that

$$
v_{x}-u_{y}=\Psi_{x x}+\Psi_{y y}=\nabla^{2} \Psi
$$


Hence, in terms of the streamfunction $\Psi$, equation (2.32) is written as

$$
\nabla^{2} \Psi_{t}+\Psi_{x} \nabla^{2} \Psi_{y}-\Psi_{y} \nabla^{2} \Psi_{x}+\beta \Psi_{x}-\nu \nabla^{4} \Psi=0
$$

This equation is known as the barotropic vorticity equation. It describes the fluid motion on a beta-plane in terms of the stream function $\Psi$ and the quantity $\xi=\nabla^{2} \Psi$ which is known as the vorticity. The vorticity $\xi$ measures the amount of rotation that the fluid undergoes in the $x y$ plane and is given by the $z$-component of the curl of $\vec{u}$

$$
\xi=\hat{z} \cdot \nabla \times \vec{u}=v_{x}-u_{y}=\Psi_{x x}+\Psi_{y y}=\nabla^{2} \Psi
$$

where $\hat{z}$ is the unit vector in the $z$ direction. Hence, in terms of $\xi$ and $\Psi$, equation (2.34) is simply,

$$
\xi_{t}+\Psi_{x} \xi_{y}-\Psi_{y} \xi_{x}+\beta \Psi_{x}-\nu \nabla^{2} \xi=0
$$

Equation (2.36) can be written as

$$
\frac{D \xi}{D t}=-\beta v+\nu \nabla^{2} \xi
$$

If there is no rotation, i.e. $\beta \approx 0$, equation (2.37) resembles the energy conservation equation. In the context of rotating flows $\xi$ is called the potential vorticity or the relative vorticity. The total vorticity $f_{o}+\beta y+\xi$ is called the absolute vorticity. Equation (2.37) represents the conservation of potential vorticity. 


\subsubsection{The non-dimensional barotropic vorticity equation}

Throughout this thesis we will work with non-dimensional variables and parameters. In this section we describe the process of nondimensionalizing the governing equation. To indicate that a variable or a parameter is dimensional, we put an asterisk $(*)$ on it, so that the dimensional barotropic voroticity equation (2.34) is written as,

$$
\nabla^{* 2} \Psi_{t^{*}}^{*}+\Psi_{x^{*}}^{*} \nabla^{* 2} \Psi_{y^{*}}^{*}-\Psi_{y^{*}}^{*} \nabla^{* 2} \Psi^{*} x^{*}+\beta^{*} \Psi_{x^{*}}^{*}-\nu^{*} \nabla^{* 4} \Psi^{*}=0
$$

with $\nabla^{* 2}=\frac{\partial^{2}}{\partial x^{* 2}}+\frac{\partial^{2}}{\partial y^{* 2}}$. We define the nondimensional variables and parameters (without asterisks) by: $x=\frac{x^{*}}{L_{x}}, y=\frac{y^{*}}{L_{y}}, t=\frac{t^{*} U}{L_{x}}, \Psi=\frac{\Psi^{*}}{U L_{y}}, \beta=\frac{\beta^{*}}{B}$ and $\nu=\frac{\nu^{*}}{\mathcal{V}}$ where $L_{x}$ and $L_{y}$ are typical length scales in the zonal (west-east) and meridional (south-north) directions respectively. For the beta-plane approximation to be valid we can only consider a narrow range of latitudes, so $L_{y}$ must be relatively small, i.e. $L_{y}<<L_{x}$. $B$ is a typical scale for the planetary vorticity gradient in the meridional direction, $U$ a typical velocity and $\mathcal{V}$ is the scale of the kinematic viscosity. The dimensional linear differential operators in terms of the nondimensional parameters are thus given by:

$$
\frac{\partial}{\partial t^{*}}=\frac{U}{L_{x}} \frac{\partial}{\partial t}, \frac{\partial}{\partial x^{*}}=\frac{1}{L_{x}} \frac{\partial}{\partial x}, \frac{\partial}{\partial y^{*}}=\frac{1}{L_{y}} \frac{\partial}{\partial y}
$$

and

$$
\nabla^{* 2}=\frac{1}{L_{y}^{2}}\left(\delta \frac{\partial^{2}}{\partial x^{2}}+\frac{\partial^{2}}{\partial y^{2}}\right)=\frac{1}{L_{y}^{2}} \nabla^{2}
$$

where $\delta=\frac{L_{y}^{2}}{L_{x}^{2}}$ is the square of the aspect ratio. Since we want $L_{y}<<L_{x}$ we assume that $\delta<<1$. In terms of the nondimensional quantities, equation (2.38) 
becomes

$$
\nabla^{2} \Psi_{t}+\Psi_{x} \nabla^{2} \Psi_{y}-\Psi_{y} \nabla^{2} \Psi_{x}+\beta B \frac{L_{y}{ }^{2}}{U} \Psi_{x}-\nu \mathcal{V} \frac{L_{x}}{U L_{y}{ }^{2}} \nabla^{4} \Psi=0
$$

This suggests that we let

$$
B=\frac{U}{L_{y}^{2}}
$$

and

$$
\mathcal{V}=\frac{U L_{y}^{2}}{L_{x}}
$$

which means the nondimensional kinematic viscosity is given by

$$
\nu=\frac{\nu^{*}}{\mathcal{V}}=\frac{1}{R e}=\nu \frac{L_{x}}{U L_{y}{ }^{2}}
$$

while the nondimensional gradient of the planetary voricity in $y$ direction is

$$
\beta=\frac{\beta^{*}}{B}=\beta \frac{L_{y}^{2}}{U}
$$

This gives the nondimensional barotropic vorticity equation

$$
\nabla^{2} \Psi_{t}+\Psi_{x} \nabla^{2} \Psi_{y}-\Psi_{y} \nabla^{2} \Psi_{x}+\beta \Psi_{x}-\nu \nabla^{4} \Psi=0
$$

Clearly this equation is similar to the dimensional one (2.34), with the only difference being that the Laplacian operator has changed, $\nabla^{2}=\delta \frac{\partial^{2}}{\partial x^{2}}+\frac{\partial^{2}}{\partial y^{2}}$.

To describe wave propagation, we write the streamfunction $\Psi$ as the sum of 2 terms, one corresponding to the basic background fluid flow and the other corresponding to the perturbation. In terms of the dimensional variables the 
streamfunction is given by

$$
\Psi^{*}(x, y, t)=\bar{\psi}^{*}(y)+\psi^{*}(x, y, t)
$$

where $\bar{\psi}(y)$ is the zonal mean background flow according to (1.2). We make this equation nondimensional by defining $\Psi^{*}=\frac{\Psi}{U L_{y}}, \bar{\psi}^{*}=\frac{\bar{\psi}}{U L_{y}}$ and $\psi^{*}=\frac{\psi}{\varphi}$ where $\varphi$ is the amplitude of the wave perturbation streamfunction at the source. So in terms of the nondimensional quantities, the total streamfunction is

$$
\Psi=\bar{\psi}+\frac{\varphi}{U L_{y}} \psi
$$

Now let

$$
\varepsilon=\frac{\varphi}{U L_{y}}
$$

This parameter $\varepsilon$ gives a measure of the disturbance at the source relative to the magnitude of the background flow. We will see that $\varepsilon$ characterizes the nonlinearity of the governing equations. So the total streamfunction $\Psi$ is now written as

$$
\Psi(x, y, t)=\bar{\psi}(y)+\varepsilon \psi(x, y, t)
$$

where $\psi(x, y, t)$ is the perturbation function and $\bar{\psi}(y)$ is the initial zonal mean streamfunction and is given by

$$
\bar{\psi}(y)=\left[\frac{1}{L} \int_{0}^{L} \Psi(x, y, t) d x\right]_{t=0}
$$

where $L$ is a suitable chosen nondimensional length in the zonal direction. In the problem that we study here where the waves are generated by a zonally periodic 
forcing at some specified latitude, the appropriate choice of $L$ is the wavelength $\frac{2 \pi}{k_{o}}$ where $k_{o}$ is the wavenumber at the source.

We also write the total vorticity as $\xi(x, y, t)=\bar{\zeta}(y)+\varepsilon \zeta(x, y, t)$ where $\zeta(x, y, t)=\nabla^{2} \psi(x, y, t)$ is the perturbation vorticity and $\bar{\zeta}(y)$ represents the initial zonal mean vorticity. If we make use of equation (2.48) and substitute for $\Psi$ and $\xi$ in the equation (2.36), rearranging the terms yields

$$
\zeta_{t}+\bar{u} \zeta_{x}+\varepsilon\left(\psi_{x} \zeta_{y}-\psi_{y} \zeta_{x}\right)+\left(\beta-\bar{u}^{\prime \prime}\right) \psi_{x}-\nu \nabla^{2} \zeta=0
$$

where $\bar{u}(y)=-\bar{\psi}^{\prime}(y)$ is the initial zonal mean velocity.

In the troposphere and stratosphere, background zonal wind speeds are generally around 10-50 $\mathrm{ms}^{-1}$ while wave perturbations have amplitudes $\sim 1 \mathrm{~ms}^{-1}$. So the nondimensional parameter $\varepsilon$ would be $\lesssim 0.1$. We are therefore justified in considering $\varepsilon$ to be a small parameter. This allows us to linearize (2.50) and make use of linear and weakly nonlinear theories. Also in geophysical flows the viscosity is generally weak enough to justify setting the nondimensional viscous parameter $\nu$ to zero. If we set $\varepsilon=0$ and $\nu=0$ in (2.50), we obtain the linearized inviscid barotropic vorticity equation.

$$
\left[\frac{\partial}{\partial t}+\bar{u} \frac{\partial}{\partial x}\right] \nabla^{2} \psi+\left(\beta-\bar{u}^{\prime \prime}\right) \frac{\partial \psi}{\partial x}=0
$$

\subsection{Governing equations for gravity waves}

In this section we derive the underlying equations to employ as a model for gravity waves starting from the momentum equations (2.3) and (2.5), the continuity equation (2.8) and the energy equation (2.24) in a 2 dimensional rectangular con- 
figuration with a single horizontal coordinate $x$ and a vertical coordinate $z$. We make the Boussinesq approximation which assumes that the density variation is negligible except in the buoyancy term in the vertical momentum equation, but consider the important density variation due to the temperature variation in the $z$ direction.

\subsubsection{The Momentum equation under the Boussinesq ap- proximation}

The Boussinesq approximation is defined in the classical paper by Spiegel and Veronis (1960). Under the Boussinesq approximation we suppose the total density $\varrho(x, z, t)$ is given by

$$
\varrho(x, z, t)=\varrho_{o}+\varrho^{\prime}(x, z, t)
$$

where $\varrho_{o}$ is constant reference density and $\varrho^{\prime}$ is the variation of the density from this constant value. The amplitude of the density variations over the scale height is small, this means $\varrho^{\prime}(x, z, t) \ll \varrho_{o}$. Substituting (2.52) into the momentum equations (2.3) and (2.5) and dividing both sides by $\varrho_{o}$ yields

$$
\begin{aligned}
& {\left[1+\frac{\varrho^{\prime}(x, z, t)}{\varrho_{o}}\right]\left[\frac{\partial u}{\partial t}+u \frac{\partial u}{\partial x}+w \frac{\partial u}{\partial z}\right]=-\frac{1}{\varrho_{o}} \frac{\partial p}{\partial x}+\frac{1}{\varrho_{o}} \mu \nabla^{2} u} \\
& {\left[1+\frac{\varrho^{\prime}(x, z, t)}{\varrho_{o}}\right]\left[\frac{\partial w}{\partial t}+u \frac{\partial w}{\partial x}+w \frac{\partial w}{\partial z}\right]=-\frac{1}{\varrho_{o}} \frac{\partial p}{\partial z}-\frac{\varrho}{\varrho_{o}} g+\frac{1}{\varrho_{o}} \mu \nabla^{2} w}
\end{aligned}
$$


Since $\frac{\varrho^{\prime}}{\varrho_{o}} \ll 1$, we can neglect the factor of $\frac{\varrho^{\prime}}{\varrho_{o}}$ in equations (2.53) and (2.54). In reality, $\varrho_{o}$ changes with altitude $z$, so in the gravity term in (2.54), a substitution which is commonly used is to replace $\varrho_{o}$ by the hydrostatic density $\bar{\rho}(z)$. What is the hydrostatic density? Consider the variation of the hydrostatic pressure $\bar{p}$ with altitude $z$

$$
\frac{d \bar{p}}{d z}=-\bar{\rho} g
$$

Combining equation (2.55) with the equation of state for a perfect gas(2.15) yields

$$
\frac{d \bar{\rho}}{\bar{\rho}}=-\frac{g}{\mathcal{R} T} d z
$$

Hence, the hydrostatic density is given by

$$
\bar{\rho}(z)=\varrho_{o} e^{-\frac{1}{H_{o}} z}
$$

where

$$
H_{o}=\frac{R T}{g}
$$

is called the the scale height of the atmosphere. So, $\varrho_{o}$ can be taken as the amplitude of the hydrostatic density and the hydrostatic density $\bar{\rho}$ as the initial mean flow density in the $z$ direction. Of course these assumptions are only valid if it is assumed that the density $\bar{\rho}(z)$ does not change very rapidly with altitude, i.e. the range of altitudes must be of the same order of magnitude as the scale height. Making these approximations leads to the Boussinesq equations

$$
\frac{\partial u}{\partial t}+u \frac{\partial u}{\partial x}+w \frac{\partial u}{\partial z}=-\frac{1}{\varrho_{o}} \frac{\partial p}{\partial x}+\nu \nabla^{2} u
$$


and

$$
\frac{\partial w}{\partial t}+u \frac{\partial w}{\partial x}+w \frac{\partial w}{\partial z}=-\frac{1}{\varrho_{o}} \frac{\partial p}{\partial z}-\frac{\varrho}{\bar{\rho}} g+\nu \nabla^{2} w
$$

where $\nu=\frac{\mu}{\varrho_{o}}$. Since under the Boussinesq approximation the density varies by only a small amount over the altitudes range, the continuity equation is simply given by (2.11) in rectangular coordinates $x$ and $z$. This allows us to define a streamfunction $\Psi$ by $u=-\Psi_{z}, w=\Psi_{x}$ and then combine equations (2.59) and (2.60) into a single equation for the streamfunction and the vorticity as we did for the Rossby wave configuration. We differentiate (2.59) with respect to $z$ and (2.60) with respect to $x$ and substract the first equation from the second to obtain

$$
\nabla^{2} \Psi_{t}-\Psi_{z} \nabla^{2} \Psi_{x}+\Psi_{x} \nabla^{2} \Psi_{z}+\frac{g}{\bar{\rho}} \frac{\partial \varrho}{\partial x}-\nu \nabla^{4} \Psi=0
$$

In this case, the vorticity $\xi$ is given by

$$
\xi=-\hat{y} \cdot \nabla \times \vec{u}=w_{x}-u_{z}=\Psi_{x x}+\Psi_{z z}=\nabla^{2} \Psi
$$

where $\hat{y}$ is the unit vector in the $y$ direction. So (2.61) can be written as

$$
\xi_{t}-\Psi_{z} \xi_{x}+\Psi_{x} \xi_{z}+\frac{g}{\bar{\rho}} \frac{\partial \varrho}{\partial x}-\nu \nabla^{2} \xi=0
$$

In more compact notation equation (2.63) is written as

$$
\frac{D \xi}{D t}=-\frac{g}{\bar{\rho}} \frac{\partial \varrho}{\partial x}+\nu \nabla^{2} \xi
$$




\subsubsection{The energy equation under the Boussinesq approx- imation}

For a perfect gas the energy equation is (2.24); we shall write it in terms of the density. Under the Boussinesq approximation the pressure is almost constant. So the equation of state (2.15) can be written as

$$
\varrho T \approx \text { Constant }
$$

Moreover, we can write

$$
\varrho T=\varrho_{o} T_{o}
$$

A slight change in temperature $T^{\prime}$ induces a slight change in density $\varrho^{\prime}$, in such a way $T=T_{o}+T^{\prime}$ and $\varrho=\varrho_{o}+\varrho^{\prime}$ and $\varrho^{\prime} \ll \varrho, T^{\prime} \ll T$. Making this substitution for $\varrho$ and $T$ into (2.66) yields

$$
\varrho^{\prime} T_{o}+\varrho_{o} T_{o}+\varrho^{\prime} T^{\prime}+\varrho_{o} T^{\prime}=\varrho_{o} T_{o}
$$

The third term on the left hand side is negligible compared with other terms since it is a product of perturbation quantities. Thus (2.67) becomes

$$
\varrho^{\prime} T_{o}=-\varrho_{o} T^{\prime}
$$

If we substitute for $\varrho^{\prime}$ and $T^{\prime}$ in the equation (2.68), use the fact that $\varrho-\varrho_{o}=\varrho^{\prime}$ and $T-T_{o}=T^{\prime}$, and rearrange the terms, we obtain

$$
\varrho=\varrho_{o}\left(1-\frac{1}{T_{o}}\left(T-T_{o}\right)\right)
$$


So according to (2.69), there is a linear relation between the total density $\varrho$ and the temperature $T$ and so the energy equation (2.24) can be written in terms of the density as

$$
\frac{D \varrho}{D t}=\alpha \nabla^{2} \varrho
$$

This equation can be written in terms of the streamfunction and the density as

$$
\varrho_{t}-\Psi_{z} \varrho_{x}+\Psi_{x} \varrho_{z}-\alpha \nabla^{2} \varrho=0
$$

Our gravity wave model comprises equation (2.63) coupled with equation (2.71).

\subsubsection{The nondimensional equations for $\Psi$ and $\varrho$}

We nondimensionalize equations (2.63) and (2.71) in a similar fashion to what we did for Rossby waves. We make all the variables and parameters in the equations (2.63) and (2.71) nondimensional on the basis of the typical length scales $L_{x}$ and $L_{z}$ in the horizontal and vertical directions, $U$ a typical velocity scale, $M$ a typical mass scale, the typical volume scale $V$, the typical kinematic viscosity scale $\mathcal{V}$ and the typical thermal diffusivity constant $\mathcal{A}$ in a such a way the nondimensional quantities are $x=\frac{x^{*}}{L_{x}}, z=\frac{z^{*}}{L_{z}}, t=\frac{t^{*} U}{L_{x}}, \Psi=\frac{\Psi^{*}}{U L_{z}}, g=g^{*} \frac{L_{z}}{U^{2}}$ and $\nu^{*}=\frac{\nu}{\mathcal{V}}, \rho^{*}=\rho \frac{V}{M}, \bar{\rho}=\bar{\rho}^{*} \frac{V}{M}$ and $\alpha=\frac{\alpha^{*}}{\mathcal{A}}$. The dimensional quantities are those with a superscript $*$. The scale $L_{z}$ is related to the dimensional scale height $H_{o}$ and for the Boussinesq approximation to be valid we consider that $L_{z}$ is relatively small, i.e., $L_{z}<<L_{x}$. In fact, the scales for the kinematic viscosity and the thermal diffusivity are also defined in terms of $L_{x}$ and $L_{z}, U$.

In terms of the nondimensional quantities, equations (2.63) and (2.71) re- 
spectively become

$$
\nabla^{2} \Psi_{t}-\Psi_{z} \nabla^{2} \Psi_{x}+\Psi_{x} \nabla^{2} \Psi_{z}+\frac{g}{\bar{\rho}} \frac{\partial \varrho}{\partial x}-\nu \mathcal{V} \frac{L_{x}}{U L_{z}^{2}} \nabla^{4} \Psi=0
$$

and

$$
\varrho_{t}-\Psi_{z} \varrho_{x}+\Psi_{x} \varrho_{z}-\alpha \mathcal{A} \frac{L_{x}}{U L_{z}} \nabla^{2} \varrho=0
$$

This suggests that the nondimensional kinematic viscosity and the nondimensional thermal diffusivity are given respectively by

$$
\nu=\frac{\nu^{*}}{\mathcal{V}}=\nu \frac{L_{x}}{U L_{z}^{2}}
$$

and

$$
\alpha=\frac{\alpha^{*}}{\mathcal{A}}=\alpha \frac{L_{x}}{U L_{z}}
$$

Hence, this leads to the nondimensional equations

$$
\nabla^{2} \Psi_{t}-\Psi_{z} \nabla^{2} \Psi_{x}+\Psi_{x} \nabla^{2} \Psi_{z}+\frac{g}{\bar{\rho}} \frac{\partial \varrho}{\partial x}-\nu \nabla^{4} \Psi=0
$$

and

$$
\varrho_{t}-\Psi_{z} \varrho_{x}+\Psi_{x} \varrho_{z}-\alpha \nabla^{2} \varrho=0
$$

where this time the Laplacian operator is $\nabla^{2}=\delta \frac{\partial^{2}}{\partial x^{2}}+\frac{\partial^{2}}{\partial z^{2}}$ and $\delta=\frac{L_{z}^{2}}{L_{x}^{2}}$ is the square of the aspect ratio. Since we want $L_{z}<<L_{x}$, we assume that $\delta<<1$.

To define the gravity wave perturbation we consider each fluid variable to be the sum of an initial horizontal mean part and a time dependent perturbation. We write 


$$
\begin{aligned}
\Psi(x, z, t) & =\bar{\psi}(z)+\varepsilon \psi(x, z, t) \\
\varrho(x, z, t) & =\bar{\rho}(z)+\varepsilon \rho(x, z, t)
\end{aligned}
$$

with

$$
\varepsilon=\frac{L_{z} U}{\varphi} \ll 1
$$

Substituting equations (2.78) and (2.79) into equation (2.76) and (2.77) yields

$$
\zeta_{t}+\bar{u}^{\prime} \zeta_{x}-\bar{u}^{\prime \prime} \psi_{x}+\frac{g}{\bar{\rho}} \rho_{x}+\varepsilon\left(\psi_{x} \zeta_{z}-\psi_{z} \zeta_{x}\right)-\nu \nabla^{2} \zeta=0
$$

where

$$
\zeta=\nabla^{2} \psi
$$

and

$$
\rho_{t}+\bar{u} \rho_{x}+\bar{\rho}^{\prime} \psi_{x}+\varepsilon\left(\psi_{x} \rho_{z}-\psi_{z} \rho_{x}\right)-\alpha \nabla^{2} \rho=0
$$

where prime represents differentiation with respect to $z$. We will use (2.80) and (2.82) to simulate the propagation of gravity waves. Letting $\varepsilon, \nu$ and $\alpha$ be small enough, the nonlinear and the viscosity terms can be neglected to get the linearized equations for gravity waves which are

$$
\nabla^{2} \psi_{t}+\bar{u}^{\prime} \nabla^{2} \psi_{x}-\bar{u}^{\prime \prime} \psi_{x}+\frac{g}{\bar{\rho}} \rho_{x}=0
$$

and

$$
\rho_{t}+\bar{u} \rho_{x}+\bar{\rho}^{\prime} \psi_{x}=0
$$




\subsection{Asymptotic behavior of solutions of the lin- ear problems across the critical layer}

In the linear Rossby wave problem if we assume that the solution of the linear inviscid barotropic vorticity equation (2.51) has the form $\psi(x, y, t)=\phi(y) e^{i k(x-c t)}$ then the amplitude $\phi(y)$ is given by the Rayleigh-Kuo equation

$$
\frac{d^{2} \phi}{d y^{2}}+\left[\frac{\beta}{\bar{u}-c}-\delta k_{o}^{2}\right] \phi=0
$$

which is singular if $\bar{u}(y)=c$ at some critical latitude $y=y_{c}$. The solutions for this equation (Lin 1995) can be obtained using the method of Frobenius about $y=y_{c}$ and are of the form

$$
\phi_{A}(y)=\left(y-y_{c}\right)+\frac{\beta-\bar{u}_{c}^{\prime \prime}}{2 \overline{u_{c}^{\prime \prime}}}\left(y-y_{c}\right)^{2}+\ldots
$$

and

$$
\phi_{B}(y)=1+\ldots+\frac{\beta-\bar{u}_{c}^{\prime \prime}}{2 \overline{u_{c}^{\prime \prime}}} \phi_{A} \log \left(y-y_{c}\right)+\ldots
$$

and the general solution is simply the linear combination $\phi=a \phi_{A}+b \phi_{B}$ where the constant $a$ and $b$ are determined from the boundary conditions. Below the critical latitude $y<y_{c}$ so the logarithm is expressed as $\log \left(y-y_{c}\right)=\log \left|y-y_{c}\right|+i \theta$ where $\theta$ is called the logarithmic phase shift. Hence there is a discontinuity in the solution $\phi_{B}$ across the critical layer.

This means that the streamfunction $\psi$ is discontinuous across the critical layer and the average momentum flux which has a jump discontinuity at $y=y_{c}$ would be independent of $y$ according to the Eliassen-Palm theorem (Appendix B). The singularity in equation (2.85) and the discontinuity are the results of 
having neglected the time dependence of the amplitude of the solution.

This analysis of the behavior of the disturbance across the critical layer can be generalized to the more realistic situation for which it is assumed that the streamfunction has the form $\psi(x, y, t)=\hat{\psi}(y, t) e^{i k x}$ and the amplitude varies with time. For the special case $\bar{u}=y$ and $\delta=0$ (the long-wave assumption) we can derive an asymptotic solution in terms of modified Bessel functions (Dickinison 1970 and Warn and Warn 1976). In this particular case the critical latitude is at $y_{c}=0$.

Setting $\bar{u}=y$ and $\delta=0$ and applying the Laplace transform to (2.85) and rearranging terms leads to

$$
\left(y-\frac{i s}{k}\right) \tilde{\psi}_{y y}(y, s)+\tilde{\psi}(y, s)=0
$$

where $\tilde{\psi}(y, s)$ is the Laplace transform of $\hat{\psi}(y, t)$. If at $y=y_{1}$ we apply the monochromatic forcing $\psi\left(x, y_{1}, t\right)=e^{i k_{o} x}$ so $\hat{\psi}\left(y_{1}=1, t\right)$ and $\tilde{\psi}\left(y_{1}, s\right)=\frac{1}{s}$. Solutions of (2.88) can be expressed in terms of modified Bessel functions $\mathcal{I}_{1}$ and $\mathcal{K}_{1}$ and it is required that the solution is bounded as $y \rightarrow-\infty$. We find

$$
\psi(x, y, t)=\frac{1}{2 \pi i} \int_{-i \infty}^{i \infty} \frac{e^{s t}}{s} F(s) d s
$$

where

$$
F(s)=\frac{\left(\frac{i s}{k_{o}}-y\right)^{1 / 2} \mathcal{K}_{1}\left[2\left(\frac{i s}{k_{o}}-y\right)^{1 / 2}\right]}{\left(\frac{i s}{k_{o}}-y_{1}\right)^{1 / 2} \mathcal{K}_{1}\left[2\left(\frac{i s}{k_{o}}-y_{1}\right)^{1 / 2}\right]}
$$

where $\mathcal{K}_{1}$ is the modified Bessel function of the second kind of order 1.

An asymptotic solution valid in the 'outer' region above and below the critical 
layer mainly called the outer solution and valid for large time was first correctly derived by Warn and Warn (1976) by taking the inverse Laplace transform of $\tilde{\psi}(x, y, s)$ to obtain

$$
\begin{array}{r}
\psi(x, y, t) \sim e^{-i k_{o} x} \frac{(-y)^{1 / 2} \mathcal{K}_{1}\left[(-y)^{1 / 2}\right]}{\left(-y_{1}\right)^{1 / 2} \mathcal{K}_{1}\left[2\left(-y_{1}\right)^{1 / 2}\right]}-\frac{1}{\left(y-y_{1}\right)^{1 / 2} \mathcal{K}_{1}\left[2\left(y-y_{1}\right)^{1 / 2}\right]} \frac{e^{-i k_{o} y t}}{k_{o}^{2} y t^{2}} \\
+e^{-i k_{o} x}\left(y_{1}-y\right)^{1 / 2} \mathcal{K}_{1}\left[2\left(y_{1}-y\right)^{1 / 2}\right] \frac{e^{-i k_{o} y_{1} t}}{k_{o}^{2} y t^{2}}+O\left(y^{-2} t^{-3}\right)
\end{array}
$$

$$
(-y)^{1 / 2} \mathcal{K}\left[2(-y)^{1 / 2}\right] \sim 1+\log y+(1-2 \gamma) y+\ldots
$$

where $\log y=\log |y|+i \theta$ for $y<0$ and one can show that $\theta=-\pi$. The solution inside the critical layer can be obtained using a combination of the method of matched asymptotic expansions and the method of multiple scales. Solution (2.90) exhibit the same logarithmic phase shift $\theta=-\pi$ as the steady solution (2.87) and tends to a steady solution as $t$ becomes large.

The leading order steady term of the solution is discontinuous so the averaged momentum flux is discontinous above the critical layer. In a nonlinear configuration this divergence of momentum flux results in an acceleration of the mean flow in the critical layer. The physical interpretation of this is that there is a transfer of momentum flux (and energy) from the waves to the mean flow or the waves are absorbed by the mean flow.

A similar analysis for gravity waves can be carried out. Since the propagation of gravity waves is governed by 2 equations, equations (2.83) and (2.84), it is better to combine these 2 equations into one in order to reduce the complexity of the problem. To do so, let us apply the operator $\left[\frac{\partial}{\partial t}+\bar{u} \frac{\partial}{\partial x}\right]$ to equation $(2.83)$, and $\frac{g}{\bar{\rho}} \frac{\partial}{\partial x}$ to equation (2.84) to obtain respectively 


$$
\left[\frac{\partial}{\partial t}+\bar{u} \frac{\partial}{\partial x}\right]^{2} \nabla^{2} \psi-\bar{u}^{\prime \prime}(z)\left[\frac{\partial}{\partial t}+\bar{u} \frac{\partial}{\partial x}\right] \psi_{x}+\left[\frac{\partial}{\partial t}+\bar{u} \frac{\partial}{\partial x}\right] g \frac{\rho_{x}}{\bar{\rho}(z)}=0
$$

and

$$
\frac{g}{\bar{\rho}}\left[\frac{\partial}{\partial t}+\bar{u} \frac{\partial}{\partial x}\right] \rho_{x}=N^{2} \psi_{x x}
$$

where

$$
N^{2}=-\frac{g}{\bar{\rho}} \frac{\partial \rho}{\partial z}
$$

$N$ has unit of frequency $s^{-1}$ and is called the Brunt-Väisälä frequency. Substituting for $\bar{\rho}$ into equation (2.93) yields

$$
N^{2}=\frac{g}{H_{o}}
$$

the constant Brunt-Väisälä frequency to be used in this study. Combining equations (2.91) and (2.92) yields

$$
\left[\frac{\partial}{\partial t}+\bar{u} \frac{\partial}{\partial x}\right]^{2} \nabla^{2} \psi-\bar{u}^{\prime \prime}(z)\left[\frac{\partial}{\partial t}+\bar{u} \frac{\partial}{\partial x}\right] \psi_{x}+N^{2} \psi_{x x}=0
$$

We assume that the solution of this equation has the form $\psi(x, z, t)=\phi(z) e^{i k_{o}(x-c t)}$. Miles (1961) found that the independent linear solutions to the steady amplitude equation that is given by the Taylor Goldstein equation

$$
\frac{d^{2} \phi}{d z^{2}}-\left[\frac{N^{2}}{(\bar{u}-c)^{2}}+\frac{\bar{u}^{\prime \prime}}{(\bar{u}-c)}+\delta k_{o}^{2}\right] \phi=0
$$

which are

$$
\begin{aligned}
& \phi_{A}(z) \sim\left(z-z_{c}\right)^{1 / 2+i \gamma} \\
& \phi_{B}(z) \sim\left(z-z_{c}\right)^{1 / 2-i \gamma}
\end{aligned}
$$


are valid in the outer region above and below the critical level and are discontinuous across the critical layer with a phase change of $-\pi$. In the case where $\bar{u}=z$ and $\delta=0$ the solutions are given exactly by the expressions in (2.97) and (2.98).

Booker and Bretherton (1967) in their study generalized this problem by assuming $\psi(x, z, t)=\hat{\psi}(z, t) e^{i k x}$ and did apply a monochromatic forcing $e^{i k_{o} x}$ at $z=z_{1}$. They concluded that there is wave absorption by the mean flow across the critical layer as in the linear Rossby wave problem. In the present study the main question is "What actually happen if there is not a critical layer in the flow?".

\subsection{Exact expressions for transient Rossby and gravity waves}

In our numerical solution we use a mean flow with no critical latitude or critical level. The simplest way to do so is to set $\bar{u}=$ constant. For this type of the mean flow velocity Nadon and Campbell (2007) derived an exact expression for the transient gravity wave for a monochromatic forcing $e^{i k_{o} x}$ applied at lower boundary $z=z_{1}$. Seeking a solution of the form $\psi(x, z, t)=\hat{\psi}(z, t) e^{i k_{o} x}$ and taking the long-wave limit $\delta=0$, they obtained the amplitude equation

$$
\left[\frac{\partial}{\partial t}+i k_{o} \bar{u}\right] \phi_{z z}(z, t)-N^{2} k_{o}^{2} \phi(z, t)=0
$$


which they solved by taking a Laplace transform in time. For a wave with upward group velocity and positive wavenumber $k_{o}>0$ they got the solution

$$
\begin{array}{r}
\psi(x, z, t)=e^{i k_{o} x} e^{-\frac{i N\left(z-z_{1}\right)}{\bar{u}-c}}-e^{i k(x-\bar{u} t)} \sum_{n=1}^{\infty}\left(\frac{\sqrt{N\left(z-z_{1}\right)}}{i(\bar{u}-c) \sqrt{k t}}\right)^{n} \mathcal{J}_{n}\left(2 \sqrt{N k_{o}\left(z-z_{1}\right) t}\right) \\
+ \text { c.c. }
\end{array}
$$

where $\mathcal{J}_{n}$ are Bessel functions of the first type of order $n$. This series solution is an example of a general class of series called Neumann series whose elements are Bessel functions of increasing order. Neumann series and their relations to Laplace integrals are discussed in the recent work of Pogany and Suli (2009).

If the time $t \rightarrow \infty$ the second term of (2.100) vanishes, the remaining term purely periodic in $x$ and $z$ and is the solution of the Taylor Goldstein equation (2.96). We note that $\psi(x, z, t)$ is continuous and is finite as the time $t$ approaches infinity. By using the series expansion for the Bessel function

$$
\mathcal{J}_{n}(\gamma)=\sum_{j=0}^{\infty}(-1)^{j} \frac{\left(\frac{\gamma}{2}\right)^{2 j+n}}{j !(n+j) !}
$$

it is easy to verify that the solution (2.100) is zero at the time $t=0$.

A similar approach to that of Nadon and Campbell (2007) can be utilized in order to derive an exact expression for the transient Rossby wave in the long-wave limit $\delta=0$. If we seek a solution of the form $\psi(x, y, t)=\phi(y, t) e^{i k_{o} x}$ with the monochromatic forcing being applied at the upper boundary of the rectangular domain $y=y_{1}$ and then solve the linearized inviscid barotropic equation by taking a Laplace transform in time we find that the Laplace transform of the 
amplitude $\phi(y, t)$ is

$$
\tilde{\phi}(y, s)=\frac{e^{ \pm i \sqrt{\frac{i k_{0} \beta}{s+i k_{0} \bar{u}}}}}{s}
$$

$\phi(y, t)$ is computed in Appendix D by inverting the Laplace transform and is given by

$\phi(y, t)=e^{-i \sqrt{\frac{\beta}{\bar{u}}}(y-y 1)}-2 e^{-i k_{o} \bar{u} t} \sum_{l=0}^{\infty} \sum_{j=0}^{\infty} \frac{1}{\left(i k_{o} \bar{u}\right)^{l+1}} \frac{\left(-i k_{o} \beta\right)^{l+j+1}\left(y-y_{1}\right)^{2 l+2 j+2} t^{j}}{(2 l+2 j+2) ! j !}$

Hence the streamfunction $\psi(x, y, t)$ is given by

$$
\begin{array}{r}
\psi(x, y, t)=-2 e^{i k_{o}(x-\bar{u} t)} \sum_{l=0}^{\infty} \sum_{j=0}^{\infty} \frac{1}{\left(i k_{o} \bar{u}\right)^{l+1}} \frac{\left(-i k_{o} \beta\right)^{l+j+1}\left(y-y_{1}\right)^{2 l+2 j+2} t^{j}}{(2 l+2 j+2) ! j !} \\
+e^{i k_{o} x} e^{-i \sqrt{\frac{\beta}{u}}(y-y 1)}+c . c(2.1
\end{array}
$$

It is shown in Appendix D that the transient part of $\phi(y, t)$ is convergent and bounded, the steady term is periodic in $x$ and $y$ and is the solution of the Rayleigh-Kuo equation (2.85). The streamfunction $\psi(x, y, t)$ is continuous and approaches a finite value as the time $t$ approaches infinity. So in the configuration in which there is no critical latitude the exact expression for the transient gravity wave (2.100) and that for the transient Rossby (2.103) do not have singularities. Hence the waves propagate all the way to the outflow boundary and so it is important to correctly implement a nonreflecting boundary condition or radiation condition at the outflow boundary. 


\section{Chapter 3}

\section{The derivation of the radiation}

\section{conditions}

In this chapter we describe and derive different boundary conditions that can be applied at the outflow boundary of each of our wave propagation configurations. The simplest one is to let the streamfunction vanish, $\Psi=0$, at the outflow boundary. In a configuration where there is a critical layer, the asymptotic solutions described in section 2.4 and numerical simulations show that there is not much transmission of wave activity beyond the critical layer. In the linear case, the waves are absorbed at the critical layer and do not reach the outflow boundary; in the nonlinear case there is wave reflection and absorbtion but not much transmission. It is therefore reasonable to apply a zero boundary condition at the outflow boundary.

If the mean flow profile is such that there is no critical layer, then the waves propagate all the way to the outflow boundary in both the linear and the nonlinear problems, and therefore a zero boundary condition is not appropriate in that

case and a non-reflecting boundary condition is needed. A simple non-reflecting 
boundary condition can be derived from the steady linearized equations. A timedependent radiation condition for Rossby waves was developed by Béland and Warn (1975) and for gravity waves by Campbell and Maslowe (2003). In this chapter we describe these boundary conditions. So in total 3 types of boundary conditions will be implemented, the zero, the steady and the time-dependent boundary conditions. For nonlinear problems, we also derive a nonlinear correction to the linear time-dependent boundary conditions.

\subsection{Steady radiation condition}

\subsubsection{Steady radiation condition for Rossby waves}

In order to derive the steady radiation condition, let us consider the streamfunction $\psi(x, y, t)$ to be a plane wave with a single wavenumber $k_{o}$ in the $x$-direction, for which the amplitude is a function of $y$ only. The fixed wavenumber $k_{o}$ and the phase speed $c$ are specified by imposing a boundary condition $e^{i k_{o}(x-c t)}$ at the forced boundary. So the streamfunction is simply

$$
\psi(x, y, t)=\phi(y) e^{i k_{o}(x-c t)}+c . c .
$$

where the amplitude $\phi$ is complex. Now, let us make a substitution for $\psi(x, y, t)$ into equation (2.51) to get the Rayleigh-Kuo equation (2.85).

If $\bar{u}$ is constant then the equation (2.85) can be written as

$$
\left(\frac{d}{\partial y}-i l\right)\left(\frac{d}{\partial y}+i l\right) \phi(y)=0
$$


where

$$
l^{2}=\frac{\beta}{\bar{u}-c}-\delta k_{o}^{2}
$$

If $\frac{\beta}{\bar{u}-c}-\delta k_{o}^{2}<0$ then the solutions grow or decay exponentially with $y$ and there is no wave propagation. We only consider the case $\frac{\beta}{\bar{u}-c}-\delta k_{o}^{2}>0$ which allow wave propagation.

The steady radiation condition is

$$
\frac{d \phi}{d y} \pm i l \phi=0
$$

at the outflow boundary $y=y_{2}$. The choice of the sign in (3.4) is determined by the sign of the $y$-component $C_{g y}$ of the group velocity. If the sign of $C_{g y}$ is positive, the waves propagate upward. If it is negative, then the waves propagate downward. In our Rossby wave configuration the forcing is at the upper boundary of the rectangular domain; so the waves propagate downward. Therefore we need $C_{g y}$ to be negative. The $y$-component of the group velocity is (see Appendix A)

$$
C_{g y}=\frac{2 \beta l k}{\left(\delta k^{2}+l^{2}\right)^{2}}
$$

For the solution with the wavenumber $+l$ the $y$-component of the group velocity is

$$
C_{g y}(l)=\frac{\partial \omega}{\partial l}=+\frac{2 \beta l k}{\left(\delta k^{2}+l^{2}\right)^{2}}
$$

while for the solution with wavenumber $-l$,

$$
C_{g y}(-l)=\frac{\partial \omega}{\partial(-l)}=-\frac{2 \beta l k}{\left(\delta k^{2}+l^{2}\right)^{2}}
$$


If $k>0, l>0$ then $C_{g y}(l)>0$ and $C_{g y}(-l)<0$. Thus in order to have a downward propagating wave with $C_{g y}<0$ we must choose $-l$ which corresponds to $\phi \sim e^{-i l y}$. If $k>0, l<0$ then we must choose $+l$ which corresponds to $\phi \sim e^{i l y}$. If $k<0$ then we choose $+l$ for $l>0$ and $-l$ for $l<0$. Hence, the steady boundary condition is given by

$$
\frac{d \phi}{d y}+\operatorname{sgn}(k) i|l| \phi=0
$$

This steady radiation condition can be used in an approximate sense in cases where $\bar{u}(y)$ is approximately constant near the outflow boundary, e.g if $\bar{u}=$ $\tanh \left(y-y_{c}\right)$ for $y_{1}>y>y_{2}$ with the radiation condition at $y=y_{2}$ then $\bar{u}\left(y_{2}\right) \approx-1$ provided $y_{c}-y_{2}$ is large enough and we can compute $l$ as

$$
l^{2} \approx \frac{\beta}{\bar{u}\left(y_{2}\right)-c}-\delta k_{o}^{2}
$$

In a configuration where the perturbation comprises a spectrum of wavenumbers

$$
\psi(x, y, t)=\sum_{k=-K / 2}^{k=K / 2} \hat{\phi}_{k}(y) e^{i \kappa(x-c t)}
$$

where $\kappa=k_{o} k$ with $k=-K / 2, \cdots K / 2$, we can apply (3.8) for each wavenumber $\kappa$ as

$$
\frac{d \hat{\phi}_{k}}{d y}+\operatorname{sgn}(k) i|l| \hat{\phi}_{k}=0
$$

where

$$
l^{2}(k) \approx \frac{\beta}{\bar{u}\left(y_{2}\right)-c}-\delta \kappa^{2}(k)
$$


In the section (3.2), we will assume the amplitude of the streamfunction depends upon on time, we will have to use the Laplace transform in order to derive an appropriate boundary condition and this will leads to a Laplace convolution integral.

\subsubsection{Steady radiation condition for gravity waves}

To derive the steady radiation condition for gravity waves we proceed in a similar way to Rossby wave case; we only have to replace $y$ by $z$ in (3.1) and express $\psi$ as

$$
\psi(x, z, t)=\phi(z) e^{i k_{o}(x-c t)}+c . c .
$$

where $\phi$ is complex with imposed boundary condition $e^{i k_{o}(x-c t)}$ at $z=z_{1}$. Substituting $\psi$ into equation (2.95) yields the Taylor Goldstein equation (2.96). If $\bar{u}$ is constant then equation (2.96)

$$
\left(\frac{d}{\partial z}-i m\right)\left(\frac{d}{\partial z}+i m\right) \phi(z)=0
$$

where

$$
m^{2}=\frac{N^{2}}{\left(\bar{u}\left(z_{2}\right)-c\right)^{2}}-\delta k_{o}^{2}>0
$$

We consider the case $\frac{N^{2}}{\left(\bar{u}\left(z_{2}\right)-c\right)^{2}}-\delta k_{o}^{2}>0$ which allows wave propagation. If $\frac{N^{2}}{\left(\bar{u}\left(z_{2}\right)-c\right)^{2}}-\delta k_{o}^{2}<0$ then the solutions grow or decay exponentially with $z$ and therefore there is no wave propagation as in the Rossby wave problem.

So the radiation condition is

$$
\frac{d \phi}{d z} \pm i m \phi=0
$$


at the outflow boundary $z=z_{2}$. The sign in (3.16) depends on the sign of the $z$-component $\left(C_{g z}\right)$ of the group velocity. In the gravity wave case the forcing is applied at the lower boundary, the waves propagate upward. So $C_{g z}$ needs to be positive. The $z$-component of the group velocity is (see Appendix A)

$$
C_{g z}=\frac{\partial \omega}{\partial m}= \pm \frac{N k m}{\left(\delta k^{2}+m^{2}\right)^{3 / 2}}
$$

It is not easy to figure out if $C_{g z}$ is either positive or negative by inspecting (3.17) because of the duality of the \pm signs. One can use the expression for the $x$-component of the phase velocity to get rid of these signs (Booker and Bretherton 1967). The $x$-component of the phase velocity is (see Appendix A) given by

$$
c=C_{x}=\bar{u} \mp \frac{N}{\left(\delta k^{2}+m^{2}\right)^{1 / 2}}
$$

From (3.18) one can show that

$$
\left(\frac{C_{x}-\bar{u}}{N}\right)^{3}=\mp \frac{1}{\left(\delta k^{2}+m^{2}\right)^{3 / 2}}
$$

In our configuration $c=C_{x}=0$. Combining (3.17) and (3.19) yields

$$
C_{g z}=\frac{(c-\bar{u})^{3} m k}{N^{2}}
$$

The vertical wavenumber $m$ defined by (3.15) can be either positive or negative.

Now let us define $m$ so that $\operatorname{sgn}(m)=\operatorname{sgn}(\bar{u}-c)$. For the solution with wavenumber $m$ which has the same sign as $\bar{u}-c, C_{g z}$ is given by (3.20). For the 
solution with wavenumber $-m$ which has the opposite sign to $\bar{u}-c$,

$$
C_{g z}=\frac{\partial \omega}{\partial(-m)}=-\frac{(c-\bar{u})^{3} m k}{N^{2}}
$$

So if $k>0, C_{g z}(m)<0$ and $C_{g z}(-m)>0$ and so for upward propagation we should choose $-m$. If $k<0$ then we must choose $+m$. Hence, the radiation condition is

$$
\frac{d \phi}{d z}+\operatorname{sgn}(k) i|m| \phi=0
$$

Again we can use this radiation condition in an approximate sense for a mean flow configuration that is approximately constant near the outflow boundary e.g. $\bar{u}=\tanh \left(z-z_{c}\right)$. This can be generalized to a configuration where there is a spectrum of wavenumbers, $\kappa(k)$ with $k=-K / 2, \cdots, K / 2 . \psi$ in this case is given by

$$
\psi(x, z, t)=\sum_{k=-K / 2}^{k=K / 2} \hat{\phi}_{k}(z) e^{i \kappa(x-c t)}
$$

We can apply (3.22) for each wavenumber $\kappa$ as

$$
\frac{d \hat{\phi}_{k}}{d z}+\operatorname{sgn}(k) i|m(k)| \hat{\phi}_{k}=0
$$

where

$$
m^{2}(k) \approx \frac{N^{2}}{\left(\bar{u}\left(z_{2}\right)-c\right)^{2}}-\delta \kappa^{2}(k)
$$




\subsection{Time-dependent linear radiation conditions}

\subsubsection{Time-dependent linear radiation condition for Rossby waves}

The time-dependent radiation condition for this configuration was derived by Béland and Warn (1975). Again it is assumed that $\bar{u}$ is constant near the outflow boundary, but we now consider a solution where the amplitude changes with time. First, we are going to derive the boundary condition for a wave comprising a single zonal wavenumber $k_{o}$ and next generalize it to a configuration where there is a spectrum of wavenumbers by applying the Fourier transform. In order to do so, let us consider $\psi(x, y, t)$ is given by

$$
\psi(x, y, t)=\hat{\psi}(y, t) e^{i k_{o} x}+\text { c.c. }
$$

$\psi$ is no longer a plane wave because its amplitude $\hat{\psi}(y, t)$ is a function of time. To take into account time dependence of $\hat{\psi}$, we take the Laplace transform to (2.50) and (3.26), apply the condition of the initial zero vorticity $\hat{\psi}_{y y}(y, 0)=0$ and then combine these 2 equations to yield

$$
\left[s+i k_{o} \bar{u}\right]\left[-\delta k_{o}^{2}+\frac{\partial^{2}}{\partial y^{2}}\right] \tilde{\psi}(y, s)+i k_{o} \beta \tilde{\psi}(y, s)=0
$$

where $\tilde{\psi}$ is the Fourier-Laplace transform of $\psi$. Rearranging terms yields

$$
\tilde{\psi}_{y y}-\left[\delta k_{o}^{2}-\frac{i k_{o} \beta}{\left(s+i k_{o} \bar{u}\right)}\right] \tilde{\psi}=0
$$

i.e. the Rayleigh-Kuo equation with complex phase speed $c=-\frac{i s}{k_{o}}$. 
If $\bar{u}$ is constant, one can write (3.28) as

$$
\left[\frac{\partial}{\partial y}+H(s)\right]\left[\frac{\partial}{\partial y}-H(s)\right] \tilde{\psi}(y, s)=0
$$

where $H(s)$ is the square root of the expression in the square brackets in the equation (3.28) and is given by

$$
H(s)=\delta^{1 / 2} k_{o}\left[\frac{\left(s+i k_{o} \bar{u}\right)-\frac{i \beta}{\delta k_{o}}}{\left(s+i k_{o} \bar{u}\right)}\right]^{1 / 2}
$$

with $\mathcal{R}[H(s)]>0$. Using group velocity arguments we get a bounded solution as $y \rightarrow-\infty$ by keeping the term with the negative sign in front $H(s)$, then

$$
\left[\frac{\partial}{\partial y}-H(s)\right] \tilde{\psi}(y, s)=0
$$

The inverse Laplace transform of $H(s)$ can be found from tables (see for example Abramowitz and Stegun 1964) and is given by

$$
h(t)=\frac{\beta}{2 \delta^{1 / 2}} e^{i k_{o}\left(1+\frac{\beta}{2 \delta k_{o}^{2}}\right) t}\left[\mathcal{J}_{1}\left(\frac{\beta t}{2 \delta k_{o}}\right)+i \mathcal{J}_{o}\left(\frac{\beta t}{2 \delta k_{o}}\right)\right]+\delta^{1 / 2} k_{o} \delta(t-0),
$$

where $\delta(t-0)$ is the delta function and $\mathcal{J}_{o}, \mathcal{J}_{1}$ are Bessel functions of order zero and one respectively. If we invert the Laplace transform, equation(3.31) becomes

$$
\frac{\partial}{\partial y} \hat{\psi}(y, t)-h(t) * \hat{\psi}(y, t)=0
$$


where

$$
h(t) * \hat{\psi}(y, t)=\mathcal{L}^{-1}[H(s) \tilde{\psi}(y, s)]=\int_{0}^{t} \hat{\psi}(y, \tau) h(t-\tau) d \tau
$$

is the Laplace convolution integral of $h(t)$ and $\hat{\psi}(x, y, t)$. So at the boundary, with a single wavenumber, $\hat{\psi}(x, y, t)$ satisfies

$$
\frac{\partial}{\partial y} \hat{\psi}(y, t)-\delta^{1 / 2} k_{o} \psi(\hat{y}, t)=\int_{0}^{t} \psi(\hat{y}, \tau) \mathcal{H}(t-\tau) d \tau
$$

where

$$
\begin{array}{r}
\mathcal{H}(t)=\frac{\beta}{2 \delta^{1 / 2}} e^{i k_{o}\left(1+\frac{\beta}{2 \delta k_{o}^{2}}\right) t}\left[\mathcal{J}_{1}\left(\frac{\beta t}{2 \delta k_{o}}\right)+i \mathcal{J}_{o}\left(\frac{\beta t}{2 \delta k_{o}}\right)\right] \\
=h(t)-\delta^{1 / 2} k_{o} \delta(t-0)
\end{array}
$$

In a configuration where there is a spectrum of wavenumbers

$$
\psi(x, y, t)=\sum_{k=-K / 2}^{k=K / 2} \hat{\psi}_{k}(y, t) e^{i k_{o} k x}
$$

and for each wavenumber $\kappa \hat{\psi}$ satisfies

$$
\frac{\partial}{\partial y} \hat{\psi}_{k}(y, t)-\delta^{1 / 2} \kappa \hat{\psi}_{k}(y, t)=\int_{0}^{t} \hat{\psi}_{k}(y, \tau) \mathcal{H}(\kappa, t-\tau) d \tau
$$

where $\mathcal{H}=\mathcal{H}(\kappa, t)$ is obtained by simply replacing $k_{o}$ by $\kappa$ in (3.36).

For the special case of long waves that we are considering here where $\delta \ll 1$, 
$H$ could be approximated by

$$
H(\kappa, s) \approx \sqrt{\frac{i \kappa \beta}{s+i \kappa \bar{u}}}
$$

and its inverse Laplace transform is simply given by

$$
h(\kappa, t) \approx \sqrt{\frac{i \kappa \beta}{\pi t}} e^{-i \kappa \bar{u} t}
$$

Hence, an approximation to equation (3.38) is in this case given by

$$
\frac{\partial}{\partial z} \hat{\psi}_{k}(z, t)-\sqrt{\frac{i \kappa \beta}{\pi}} \int_{0}^{t} \sqrt{\frac{1}{t-\tau}} \hat{\psi}_{k}(z, \tau) e^{-i \kappa \bar{u}(t-\tau)} d \tau=0
$$

\subsubsection{Time-dependent linear radiation condition for grav- ity waves}

This time-dependent radiation condition is derived for gravity waves using the linear equations (2.83) and (2.84) and was first developed by Campbell and Maslowe (2003). It is derived for the case where $\bar{u}$ is constant or approximately constant near the outflow boundary. We use equation (2.95) to derive the timedependent radiation condition. We proceed in a similar way as in the Rossby waves case. We first consider a wave with a single wavenumber $k_{o}$ for which the streamfunction is given by

$$
\psi(x, z, t)=\hat{\psi}(z, t) e^{i k_{o} x}+c . c .
$$


Substitute into (2.95) and next take the Laplace transform and apply the condition of the initial zero vorticity $\hat{\psi}_{z z}(z, 0)=0$ and $\hat{\psi}_{z z t}(z, 0)=0$ to yield

$$
\left[s+i k_{o} \bar{u}\right]^{2}\left[-\delta k_{o}^{2}+\frac{\partial^{2}}{\partial z^{2}}\right] \tilde{\psi}(z, s)-k_{o}^{2} N^{2} \psi(z, s)=0
$$

where $\tilde{\psi}(z, s)$ is the Fourier-Laplace transform of $\psi(x, z, t)$. Rearranging the terms to get

$$
\tilde{\psi}_{z z}-\left[\frac{N^{2} k_{o}^{2}}{\left(s+i k_{o} \bar{u}\right)^{2}}+\delta k_{o}^{2}\right] \tilde{\psi}=0
$$

which is the Taylor-Goldstein equation with complex phase speed $c=\frac{i s}{k_{o}}$. Moreover let us rewrite equation (3.44) as

$$
\left[\frac{\partial}{\partial z}+H(s)\right]\left[\frac{\partial}{\partial z}-H(s)\right] \tilde{\psi}(z, s)=0
$$

where $H(s)$ is the square root of the expression in the brackets in the equation (3.44), is given by

$$
H(s)=\delta^{1 / 2} k_{o}\left[\frac{\left(s+i k_{o} \bar{u}\right)^{2}+N^{2} / \delta}{\left(s+i k_{o} \bar{u}\right)^{2}}\right]^{1 / 2}
$$

and $\operatorname{Re}[H(s)]>0$. We obtain a bounded solution for equation (3.45) as $z \rightarrow \infty$ by keeping the expression with the positive sign. Hence

$$
\tilde{\psi}_{z}+H(s) \tilde{\psi}=0
$$


It is found (Abramowitz and Stegun 1964) that its inverse Laplace transform is given by

$$
h(t)=\frac{\mathrm{N}^{2} k_{o}}{\delta^{1 / 2}} \int_{0}^{t} \frac{a}{\tau} \mathcal{J}_{1}(a \tau) d \tau e^{-i k_{o} \bar{u} t}+\delta^{1 / 2} k_{o} e^{-i k_{o} \bar{u} t} \delta(t-0),
$$

where $\delta(t-0)$ is the delta function and the constant $a=N^{2} k_{o} / \delta$. If we apply the inverse Laplace transform to (3.47), we find that $\hat{\psi}(z, t)$ satisfies

$$
\frac{\partial}{\partial z} \hat{\psi}(z, t)+\delta^{1 / 2} k_{o} \hat{\psi}(z, t)=-\int_{0}^{t} \hat{\psi}(z, \tau) \mathcal{H}(t-\tau) d \tau .
$$

where

$$
\mathcal{H}(t)=h(t)-\delta^{1 / 2} k_{o} \delta(t-0)=-\delta^{1 / 2} k_{o} g(t) e^{-i k_{o} \bar{u} t}
$$

and

$$
g(t)=\int_{0}^{t} \frac{a}{\eta} \mathcal{J}_{1}(a \eta) d \eta
$$

For a configuration where there is a spectrum of wavenumbers

$$
\psi(x, z, t)=\sum_{k=-K / 2}^{k=K / 2} \hat{\psi}_{k}(z, t) e^{i k_{o} k x}
$$

and for each wavenumber $\kappa, \hat{\psi}$ satisfies

$$
\frac{\partial}{\partial z} \hat{\psi}_{k}(z, t)+\delta^{1 / 2} \kappa \hat{\psi}_{k}(z, t)=-\int_{0}^{t} \hat{\psi}_{k}(z, \tau) \mathcal{H}(\kappa, t-\tau) d \tau
$$


where $\kappa(k)=k_{o} k$ and now $h=h(\kappa, t)$, is obtained by simply replacing $k_{o}$ by $\kappa$ in the expression for $h$, equation (3.48).

For the special case of long waves that we are considering here where $\delta \ll 1$, $H$ could be approximated by

$$
H(\kappa, s) \approx \frac{N \kappa}{s+i \kappa \bar{u}},
$$

and its inverse Laplace transform is simply given by

$$
h(\kappa, t) \approx N \kappa e^{-i \kappa \bar{u} t} .
$$

Hence, an approximation to equation (3.53) is in this case given by

$$
\frac{\partial}{\partial z} \hat{\psi}_{k}(z, t)+N \kappa e^{-i \kappa \bar{u} t} \int_{0}^{t} \hat{\psi}_{k}(z, \tau) e^{i \kappa \bar{u} \tau} d \tau=0
$$

\subsection{Time-dependent nonlinear radiation condi- tions}

All the radiation conditions derived in section 3.1 and section 3.2 are based on linearized equations. In this section we describe a way to take into account the nonlinear terms in equations (2.50) and (2.80) for Rossby and gravity waves respectively. In order to do so, we make use of weakly-nonlinear theory assuming $\psi$ is

$$
\psi \sim \psi^{(0)}+\varepsilon \psi^{(1)}+\ldots
$$


where $\psi^{(0)}$ is the solution obtained if linearized equations are used and $\psi^{(1)}$ can be obtained by substituting (3.57) into the nonlinear inviscid equations (2.50) and (2.80)-(2.82). Substituting $\psi$ into the inviscid barotropic vorticity equation (2.50) yields

$$
\begin{aligned}
& {\left[\frac{\partial}{\partial t}+\bar{u} \frac{\partial}{\partial x}\right] } \nabla^{2} \psi^{(0)}+\varepsilon\left(\psi_{x}^{(0)} \nabla^{2} \psi_{y}^{(0)}-\psi_{y}^{(0)} \nabla^{2} \psi_{x}^{(0)}\right)+\left(\beta-\bar{u}^{\prime \prime}\right) \psi_{x}^{(0)} \\
&+\varepsilon\left[\frac{\partial}{\partial t}+\bar{u} \frac{\partial}{\partial x}\right] \nabla^{2} \psi^{(1)}+\varepsilon\left(\beta-\bar{u}^{\prime \prime}\right) \psi_{x}^{(1)}+O\left(\varepsilon^{2}\right)=0
\end{aligned}
$$

We group terms according to the order of $\varepsilon$ as

$$
\begin{gathered}
O\left(\varepsilon^{0}\right):\left[\frac{\partial}{\partial t}+\bar{u} \frac{\partial}{\partial x}\right] \nabla^{2} \psi^{(0)}+\left(\beta-\bar{u}^{\prime \prime}\right) \psi_{x}^{(0)}=0 \\
O\left(\varepsilon^{1}\right):\left[\frac{\partial}{\partial t}+\bar{u} \frac{\partial}{\partial x}\right] \nabla^{2} \psi^{(1)}+\left(\beta-\bar{u}^{\prime \prime}\right) \psi_{x}^{(1)}=-\left(\psi_{x}^{(0)} \nabla^{2} \psi_{y}^{(0)}-\psi_{y}^{(0)} \nabla^{2} \psi_{x}^{(0)}\right)
\end{gathered}
$$

Equation (3.59) is just the linear inviscid barotropic vorticity equation which has solution given by (3.38). Equation (3.60) is a linear nonhomogeneous inviscid barotropic vorticity equation with the solution

$$
\psi^{(1)}=\psi_{h}^{(1)}+\psi_{p}^{(1)}
$$

where $\psi_{h}^{(1)}$ is the solution of the homogeneous linear equation and $\psi_{p}^{(1)}$ is a particular solution of the nonhomogeneous equation (3.60). 
In order to find the particular solution we substitute

$$
\psi^{(1)}(x, y, t)=\sum_{k=-K / 2}^{k=K / 2} \hat{\psi}_{k}^{(1)}(y, t) e^{i k_{o} k(x-c t)}
$$

into the nonhomogeneous equation (3.60) then take the Laplace transform of the resulting equation, apply the condition of initial zero vorticity $\hat{\psi}_{y y}(y, 0)=0$ and factorize the linear differential operator. We define

$$
g(x, y, t)=-\left(\psi_{x}^{(0)} \nabla^{2} \psi_{y}^{(0)}-\psi_{y}^{(0)} \nabla^{2} \psi_{x}^{(0)}\right)
$$

$$
\hat{g}(\kappa, y, t)=\mathcal{F}\{g(x, y, t)\}
$$

and

$$
\tilde{g}(\kappa, y, s)=\mathcal{L}\{\hat{g}\}=\mathcal{F}\{\mathcal{L}\{g\}\}
$$

To solve the nonhomogeneous equation (3.60) we take a Laplace transform and obtain

$$
\left[\frac{\partial}{\partial y}+H(\kappa, s)\right]\left[\frac{\partial}{\partial y}-H(\kappa, s)\right] \tilde{\psi}_{p}^{(1)}(y, s)=\frac{\tilde{g}(\kappa, y, s)}{s+i \kappa \bar{u}}
$$

as before where $H(\kappa, s)$ is given by

$$
H(\kappa, s)=\delta^{1 / 2} \kappa\left[\frac{(s+i \kappa \bar{u})-\frac{i \beta}{\delta \kappa}}{(s+i \kappa \bar{u})}\right]^{1 / 2}
$$

with $\mathcal{R} e(H)>0$ and $\tilde{\psi}(\kappa, y, s)$ and $\hat{\psi}(\kappa, y, t)$ are the Fourier-Laplace transform and the Fourier transform of $\psi$ respectively. Equation (3.63) can be solved by 
multiplying by an integrating factor $e^{-H(k, s) y}$ to get

$$
\frac{\partial}{\partial y}\left[e^{-H y}\left(\frac{\partial}{\partial y}-H(\kappa, s)\right) \tilde{\psi}_{p}^{(1)}(y, s)\right]=e^{-H(\kappa, s) y} \frac{\tilde{g}(\kappa, y, s)}{s+i \kappa \bar{u}}
$$

Integrating with respect to $y$ gives

$$
\left[\frac{\partial}{\partial y}-H(\kappa, s)\right] \tilde{\psi}_{p}^{(1)}(\kappa, y, s)=e^{H(\kappa, s)} \int e^{-H(\kappa, s) y} \frac{\tilde{g}(\kappa, y, s)}{s+i \kappa \bar{u}} d y
$$

which satisfies the condition of downward propagation.

Clearly (3.66) is not easy to compute in general. For the particular wavenumber $\kappa=0$ however, $H(k, s)=0$ and so equation (3.66) becomes

$$
\frac{\partial}{\partial y} \tilde{\psi}_{p}^{(1)}(0, y, s)=\int \frac{\tilde{g}(0, y, s)}{s} d y
$$

If we invert the Laplace transform, equation (3.67) becomes

$$
\frac{\partial}{\partial y} \hat{\psi}_{p}^{(1)}(0, y, t)=\int_{0}^{t} \int \hat{g}(0, y, \tau) d y d \tau
$$

This result for $\kappa=0$ could be obtained directly from (3.60) without needing a Laplace transform by setting $\kappa=0$ in (3.60) and integrating with respect to $t$ and $y$. 
For $\kappa=0$, we note that $\hat{g}(0, y, t)$ is equal to the $x$-average of $g(x, y, t)$ and so

$$
\begin{aligned}
\hat{g}(0, y, t)=\frac{k_{o}}{2 \pi} \int_{0}^{\frac{2 \pi}{k_{o}}} g(x, y, \tau) d x & =\frac{k_{o}}{2 \pi} \int_{0}^{\frac{2 \pi}{k_{o}}} J\left(\psi^{(0)}, \nabla^{2} \psi^{(0)}\right) d x \\
& =-\frac{k_{o}}{2 \pi} \int_{0}^{\frac{2 \pi}{k_{o}}} \frac{\partial^{2}}{\partial y^{2}} \psi_{x}^{(0)} \psi_{y}^{(0)} d x
\end{aligned}
$$

as shown in Appendix B.1. So the nonlinear radiation condition for the $\kappa=0$ component of the solution is given by

$$
\begin{aligned}
\frac{\partial}{\partial y} \hat{\psi}(\kappa=0, y, t)= & \int_{0}^{t} \hat{\psi}(\kappa=0, y, \tau) \mathcal{H}(\kappa=0, t-\tau) d \tau \\
& -\varepsilon \frac{k_{o}}{2 \pi} \int_{0}^{t} \int \frac{\partial^{2}}{\partial y^{2}} \int_{0}^{2 \pi / k_{o}} \psi_{x} \psi_{y} d x d y d \tau
\end{aligned}
$$

This equation can be written as

$$
\begin{array}{r}
\frac{\partial}{\partial y} \hat{\psi}(\kappa=0, y, t)=\int_{0}^{t} \hat{\psi}(\kappa=0, y, \tau) \mathcal{H}(\kappa=0, t-\tau) d \tau \\
-\varepsilon \frac{k_{o}}{2 \pi} \int_{0}^{t} \frac{\partial}{\partial y} \int_{0}^{2 \pi / k_{o}} \psi_{x} \psi_{y} d x d \tau \\
=\int_{0}^{t} \hat{\psi}(\kappa=0, y, \tau) \mathcal{H}(\kappa=0, t-\tau) d \tau+\varepsilon \int_{0}^{t} \frac{\partial}{\partial y} F d \tau
\end{array}
$$


where $F$ is the averaged momentum flux (see appendix B.1) and is given by

$$
F=-\frac{k_{o}}{2 \pi} \int_{0}^{2 \pi / k_{o}} \psi_{x} \psi_{y} d x
$$

If we apply weakly nonlinear theory to the gravity wave problem as in the Rossby wave problem we obtain the following equations according to the order of $\varepsilon$ :

$$
\begin{array}{r}
O\left(\varepsilon^{0}\right):\left[\frac{\partial}{\partial t}+\bar{u} \frac{\partial}{\partial x}\right]^{2} \nabla^{2} \psi^{(0)}-\bar{u}^{\prime \prime}(z)\left[\frac{\partial}{\partial t}+\bar{u} \frac{\partial}{\partial x}\right] \psi_{x}^{(0)}+N^{2} \psi_{x x}^{(0)}=0 \\
O\left(\varepsilon^{1}\right):\left[\frac{\partial}{\partial t}+\bar{u} \frac{\partial}{\partial x}\right]^{2} \nabla^{2} \psi^{(1)}-\bar{u}^{\prime \prime}(z)\left[\frac{\partial}{\partial t}+\bar{u} \frac{\partial}{\partial x}\right] \psi_{x}^{(1)}+N^{2} \psi_{x x}^{(1)} \\
=-\left[\frac{\partial}{\partial t}+\bar{u} \frac{\partial}{\partial x}\right] J\left(\psi^{(0)}, \nabla^{2} \psi^{(0)}\right)
\end{array}
$$

where $\psi^{(0)}$ is the solution of the linear equation $(2.95)$ and $\psi^{(1)}$ is of the form of (3.61) which the particular solution of the nonhomogeneous equation satisfies an equation analogous to (3.66). For $\kappa=0$ we can find a nonlinear correction by integrating (3.74) twice with respect to $t$ and once with respect to $z$ to yield

$$
\begin{array}{r}
\frac{\partial}{\partial z} \hat{\psi}_{p}^{(1)}(0, z, t)=\int_{0}^{t} \int \hat{g}(0, z, \tau) d z d \tau=-\int_{0}^{t} \int \mathcal{F}\left\{J\left(\psi^{(0)}, \nabla^{2} \psi^{(0)}\right)\right\} d z d \tau \\
=-\frac{k_{o}}{2 \pi} \int_{0}^{t} \int \frac{\partial^{2}}{\partial z^{2}} \int_{0}^{\frac{2 \pi}{k_{o}}} \psi_{x}^{(0)} \psi_{z}^{(0)} d x d z d t=-\frac{k_{o}}{2 \pi} \int_{0}^{t} \frac{\partial}{\partial z} \int_{0}^{\frac{2 \pi}{k_{o}}} \psi_{x}^{(0)} \psi_{z}^{(0)} d x d t
\end{array}
$$

according to Appendix B.1. Hence the component of the solution corresponding 
to the horizontal wavenumber $\kappa=0$ is given by

$$
\begin{array}{r}
\frac{\partial}{\partial z} \hat{\psi}(\kappa=0, z, t)=\int_{0}^{t} \hat{\psi}(\kappa=0, z, \tau) \mathcal{H}(\kappa=0, t-\tau) d \tau \\
-\varepsilon \frac{k_{o}}{2 \pi} \int_{0}^{t} \frac{\partial}{\partial z} \int_{0}^{2 \pi / k_{o}} \psi_{x} \psi_{z} d x d \tau \\
=\int_{0}^{t} \hat{\psi}(\kappa=0, z, \tau) \mathcal{H}(\kappa=0, t-\tau) d \tau+\varepsilon \int_{0}^{t} \frac{\partial}{\partial z} F d \tau
\end{array}
$$

where $F$ is the averaged momentum flux (see appendix B.1) and is given by

$$
F=-\frac{k_{o}}{2 \pi} \int_{0}^{2 \pi / k_{o}} \psi_{x} \psi_{z} d x
$$




\section{Chapter 4}

\section{Numerical implementation}

The numerical model for the Rossby wave propagation problem is

$$
\begin{gathered}
\zeta_{t}+\bar{u} \zeta_{x}+\left(\beta-\bar{u}^{\prime \prime}\right) \psi_{x}+\varepsilon\left(\psi_{x} \zeta_{y}-\psi_{y} \zeta_{x}\right)-\nu \nabla^{2} \zeta=0 \\
\nabla^{2} \psi=\zeta
\end{gathered}
$$

The numerical simulation is carried out on a rectangular domain defined by $x_{1} \leq x \leq x_{2}$ and $y_{1} \geq y \geq y_{2}$ with the following initial and boundary conditions

$$
\begin{gathered}
\psi(x, y, 0)=\zeta(x, y, 0)=0 \\
\psi\left(x, y_{1}, t\right)=e^{i k_{o} x}+c . c ., \quad \zeta\left(x, y_{1}, t\right)=0
\end{gathered}
$$

We set $x_{1}=0, x_{2}=2 \pi / k_{o}, y_{1}=5$ and $y_{2}=-5$, and apply periodic boundary conditions on both $\psi$ and $\zeta$ at $x_{1}$ and $x_{2}$. At the outflow boundary $y=y_{2}$, we apply the zero boundary conditions $\psi=0, \zeta=0$ or we apply one of the radiation conditions (3.11) or (3.38) for $\psi$ and then solve for $\zeta$ using (4.1). 
The numerical implementation is performed using finite difference explicit approximation and spectral methods. The spectral method is used since the boundary conditions are periodic in $x$ and therefore the fast Fourier Transform $(F F T)$ algorithm is utilized in the numerical implementation. Applying the Fourier transform in $x$ to this problem yields

$$
\begin{gathered}
\hat{\zeta}_{t}+i \kappa\left(\bar{u} \hat{\zeta}+\left(\beta-\bar{u}^{\prime \prime}\right) \hat{\psi}\right)+\varepsilon \mathcal{F}\left[\psi_{x} \zeta_{y}-\psi_{y} \zeta_{x}\right]+\nu \delta \kappa^{2} \hat{\zeta}-\nu \hat{\zeta}_{y y}=0 \\
\hat{\psi}_{y y}-\delta \kappa^{2} \hat{\psi}=\hat{\zeta}
\end{gathered}
$$

with the initial conditions

$$
\hat{\psi}(\kappa, y, 0)=\hat{\zeta}(\kappa, y, 0)=0
$$

and the boundary conditions

$$
\hat{\psi}\left(\kappa, y_{1}, t\right)=1, \hat{\zeta}\left(\kappa, y_{1}, t\right)=0
$$

and the radiation condition (3.11) or (3.38) being applied at $y=y_{2}$ to compute $\hat{\psi}$ and then using (4.5) to compute $\hat{\zeta}$.

In the linear problem there are no interactions between harmonics since $\varepsilon$ is set to zero. So simulations are carried out with a single wavenumber $k_{o}$ in the $x$ direction and the use of the spectral method is not needed. The linear problem is defined as

$$
\begin{gathered}
\zeta_{t}+\bar{u} \zeta_{x}+\left(\beta-\bar{u}^{\prime \prime}\right) \psi_{x}-\nu \nabla^{2} \zeta=0 \\
\nabla^{2} \psi=\zeta
\end{gathered}
$$


with the same boundary condition as in the nonlinear problem.

The independent variables are approximated by $x_{k}=k \Delta x, y_{j}=j \Delta y$ and $t_{n}=n \Delta t$ with $\Delta x, \Delta y$, and $\Delta t$ being the increments in $x, y$ and $t$ respectively. The discretized dependent variables in wavenumber space are given by

$$
\hat{\psi}(\kappa, y, t) \approx \hat{\psi}(k, j \Delta y, n \Delta t)=\hat{\psi}_{k, j}^{n}
$$

and

$$
\hat{\zeta}(\kappa, y, t) \approx \hat{\zeta}(k, j \Delta y, n \Delta t)=\hat{\zeta}_{k, j}^{n}
$$

with $n=1,2, \cdots, N, j=1,2, \cdots, J, k=1,2, \cdots, K$.

The simulation is done with the following spectrum of wavenumbers in the $x$ direction $\kappa=\cdots,-3 k_{o},-2 k_{o},-k_{o}, 0,+k_{o},+2 k_{o},+3 k_{o}, \cdots$, where $k_{o}$ is the wave number at the forcing (the upper boundary). The time step was set to $\Delta t=$ 0.001 and different values of $\Delta y$ were tried in the numerical experiments, $\Delta y=$ $-0.02,-0.05,-0.1,-0.2$ and results obtained from these numerical experiments were similar.

The value of $\Delta t$ required for stability could be reduced by using an implicit scheme but then there is the additional expense of inverting a matrix at each time step. The results shown here were obtained with an explicit scheme. The value of $\Delta t=0.001$ was found to be sufficiently small to maintain stability. Note that $\Delta y$ is negative since $j=1$ is the forced boundary $y=y_{1}$ and $j=J$ is the outflow boundary $y=y_{2}$ and $y_{1}>y_{2}$.

We discretize equation (4.5) using the Euler forward in time finite difference approximation and the second order central finite difference approximation in $y$ 
inside the rectangular domain to obtain

$$
\begin{array}{r}
\hat{\zeta}_{k, j}^{n+1}=\hat{\zeta}_{k, j}^{n}-i \kappa\left[\left(\beta-\bar{u}_{j}^{\prime \prime}\right) \hat{\psi}_{k, j}^{n}+\bar{u}_{j} \hat{\zeta}_{k, j}^{n}\right] \Delta t-\varepsilon \hat{J}(\psi, \zeta) \Delta t \\
+\nu\left[\hat{\zeta}_{k, j+1}^{n}+\hat{\zeta}_{k, j-1}^{n}-\hat{\zeta}_{k, j}^{n}\left(\delta(k \Delta y)^{2}+2\right)\right] \frac{\Delta t}{(\Delta y)^{2}}
\end{array}
$$

where $\hat{J}(\psi, \zeta)$ is the discrete Fourier transform $(D F T)$ of the nonlinear term (Jacobian) in the equation (4.5). We compute the Jacobian at each time level using the pseudospectral methods instead of the Fourier convolution integral which is more expensive to compute numerically, this gives

$$
\hat{J}(\psi, \zeta) \approx \mathcal{F}\left[\mathcal{F}^{-1}\left[\hat{\psi}_{x}(x, y, t)\right] \mathcal{F}^{-1}\left[\hat{\zeta}_{y}(x, y, t)\right]-\mathcal{F}^{-1}\left[\hat{\psi}_{y}(x, y, t)\right] \mathcal{F}^{-1}\left[\hat{\zeta}_{x}(x, y, t)\right]\right]
$$

where $\mathcal{F}$ and $\mathcal{F}^{-1}$ stand for the fast Fourier transform and the inverse fast Fourier transform respectively. The use of the $F F T$ and $I F F T$ algorithms allows to reduce the amount of computation from $4 N^{2}$ to $4 N \log _{2} N$ if the DFT and the IDFT are used.

The linear problem with $\varepsilon=0$ is discretized using finite difference approximation in both time and space variables to obtain

$$
\begin{array}{r}
\zeta_{k, j}^{n+1}=\zeta_{k, j}^{n}-i k_{o}\left[\left(\beta-\bar{u}_{j}^{\prime \prime}\right) \psi_{k, j}^{n}+\bar{u}_{j} \zeta_{k, j}^{n}\right] \Delta t \\
+\nu\left[\zeta_{k, j+1}^{n}+\zeta_{k, j-1}^{n}-\zeta_{k, j}^{n}\left(\delta(k \Delta y)^{2}+2\right)\right] \frac{\Delta t}{(\Delta y)^{2}}
\end{array}
$$

At each time level, we compute $\hat{\psi}$ using

$$
\hat{\psi}_{y y}-\delta \kappa^{2} \hat{\psi}=\hat{\zeta}
$$


This is discretized to obtain

$$
\hat{\psi}_{k, j+1}^{n}-\left(2+\delta \Delta y^{2} \kappa^{2}\right) \hat{\psi}_{k, j}^{n}+\hat{\psi}_{k, j-1}^{n}=(\Delta y)^{2} \hat{\zeta}_{k, j}^{n}
$$

Equation (4.17) can be written in matrix form as

$$
A \overrightarrow{\mathcal{u}}=\vec{b}
$$

where $\overrightarrow{\mathcal{U}}$, the matrix $A$ and the vector $\vec{b}$ are given respectively by

$$
\overrightarrow{\mathcal{U}}=\left(\begin{array}{c}
\hat{\psi}_{k, 1}^{n} \\
\hat{\psi}_{k, 2}^{n} \\
\hat{\psi}_{k, 3}^{n} \\
\cdots \\
\\
\hat{\psi}_{k, J-1}^{n} \\
\hat{\psi}_{k, J}^{n}
\end{array}\right)
$$

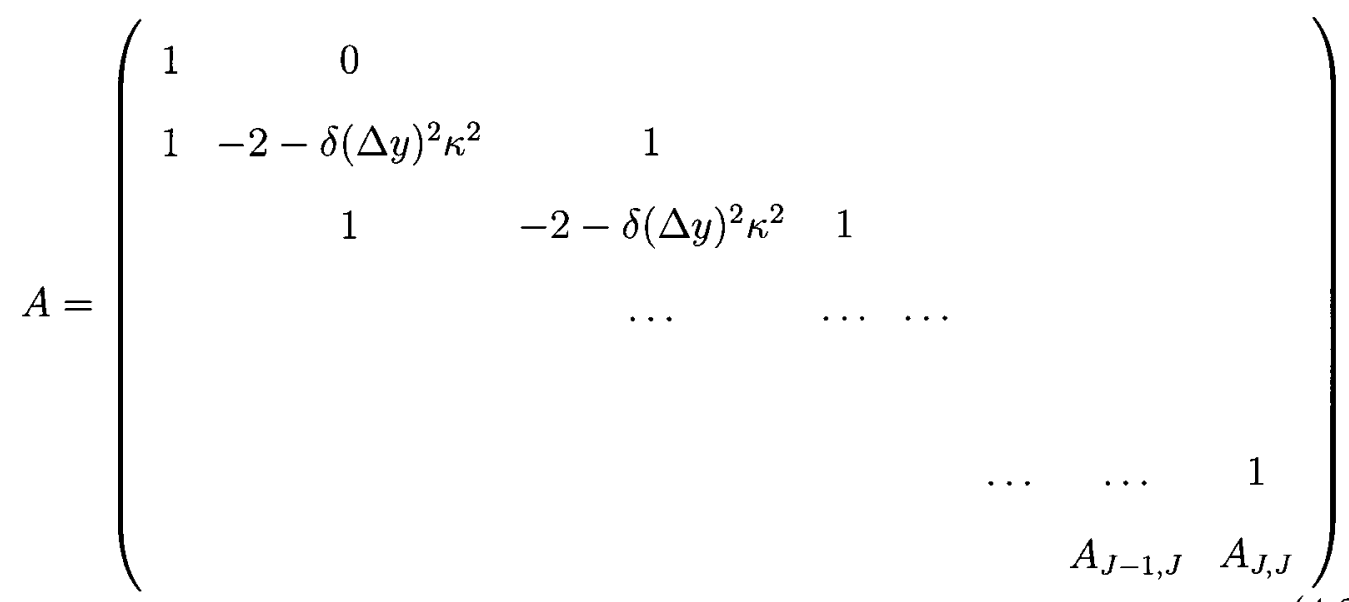


and

$$
b=(\Delta y)^{2}\left(\begin{array}{c}
\zeta_{k, 1}^{n} \\
\hat{\zeta}_{k, 2}^{n} \\
\hat{\zeta}_{k, 3}^{n} \\
\cdots \\
\\
\hat{\zeta}_{k, J-1}^{n} \\
b_{J}
\end{array}\right)
$$

Since $A$ is tridiagonal and diagonally dominant we can readily solve for $\hat{\psi}$ and then invert the transform to get $\psi$. The values of the elements in the $J^{\text {th }}$ row of the matrix $A, A_{J-1, J}$ and $A_{J, J}$, and the element $b_{J}$ in the $J^{t h}$ row of the vector $\vec{b}$ are given by the boundary condition specified.

To implement the zero boundary condition we set $A_{J-1, J}=0, A_{J, J}=1$ and $b_{J}=0$ so that $\hat{\psi}_{k, J}^{n}=0$.

The steady boundary condition is given by equation (3.8) which is discretized using the backward finite difference approximation to obtain

$$
(1+\operatorname{sgn}(k) i|l| \Delta y) \hat{\psi}_{k, J}^{n}-\hat{\psi}_{k, J-1}^{n}=0
$$

This implies that $A_{J-1, J}=0, A_{J, J}=1+\operatorname{sgn}(k) i|l| \Delta y$ and $b_{J}=0$ where $l= \pm \sqrt{\frac{\beta}{\bar{u}-c}-\delta \kappa^{2}}$. 
The numerical model for gravity waves is

$$
\zeta_{t}+\bar{u}^{\prime} \zeta_{x}-\bar{u}^{\prime \prime} \psi_{x}+\varepsilon\left(\psi_{x} \zeta_{z}-\psi_{z} \zeta_{x}\right)+g \frac{\rho_{x}}{\bar{\rho}}-\nu \nabla^{2} \zeta=0
$$

where

$$
\zeta=\nabla^{2} \psi
$$

and

$$
\rho_{t}+\bar{u} \rho_{x}+\bar{\rho}^{\prime} \psi_{x}+\varepsilon\left(\psi_{x} \rho_{z}-\psi_{z} \rho_{x}\right)-\alpha \nabla^{2} \rho=0
$$

The numerical simulation is carried out on a rectangular domain in the vertical plane which is defined by $x_{1} \leq x \leq x_{2}$ and $z_{1} \leq z \leq z_{2}$;

with the initial conditions

$$
\begin{gathered}
\psi(x, z, 0)=\zeta(x, z, 0)=0 \\
\rho(x, z, 0)=\bar{\rho}(z)=e^{-\frac{z}{H_{o}}}
\end{gathered}
$$

and the boundary conditions

$$
\psi\left(x, z_{1}, t\right)=e^{i k_{o} x}+\text { c.c., } \zeta\left(x, z_{1}, t\right)=0
$$

and the radiation condition for $\psi$ is implemented at $z=z_{2}$ and (4.23) is used to compute $\zeta$ as in the Rossby wave problem. We set $x_{1}=0, x_{2}=2 \pi / k_{o}, z_{1}=0$, $z_{2}=10$ and apply periodic boundary conditions on both $\psi, \zeta$ and $\rho$ at $x_{1}$ and $x_{2}$. 
The linear model is obtained by simply setting $\varepsilon=0$ as in Rossby wave problem and with the same boundary conditions as in the nonlinear problem. The same procedure as for Rossby waves is used for gravity waves numerical implementation. We take a Fourier transform in $x$ of equations (4.23) and (4.24), then discretize the resulting equations using finite differences in $z$ and in $t$ variables.

The difference equations are

$$
\begin{array}{r}
\hat{\zeta}_{k, j}^{n+1}=\hat{\zeta}_{k, j}^{n}-i \kappa\left[-\bar{u}_{j}^{\prime \prime} \hat{\psi}_{k, j}^{n}+\bar{u}_{j} \hat{\zeta}_{k, j}^{n}+g \frac{\hat{\zeta}_{k, j}^{n}}{\bar{\rho}_{j}}\right] \Delta t-\varepsilon \hat{J}(\psi, \zeta) \Delta t \\
+\nu\left[\hat{\zeta}_{k, j+1}^{n}+\hat{\zeta}_{k, j-1}^{n}-\hat{\zeta}_{k, j}^{n}\left(\delta(k \Delta z)^{2}+2\right)\right] \frac{\Delta t}{(\Delta z)^{2}} \\
\hat{\rho}_{k, j}^{n+1}=\hat{\rho}_{k, j}^{n}-i \kappa\left[\bar{u}_{j} \hat{\rho}_{k, j}^{n}+H_{o}^{-1} \bar{\rho}_{j} \hat{\psi}_{k, j}^{n}\right] \Delta t-\varepsilon \hat{J}(\psi, \rho) \Delta t \\
+\alpha\left[\hat{\rho}_{k, j+1}^{n}+\hat{\rho}_{k, j-1}^{n}-\hat{\rho}_{k, j}^{n}\left(\delta(k \Delta z)^{2}+2\right)\right] \frac{\Delta t}{(\Delta z)^{2}}
\end{array}
$$

where $\hat{J}(\psi, \zeta)$ and $\hat{J}(\psi, \rho)$ are the $D F T$ of the nonlinear terms (Jacobian) in the equations (4.23) and (4.25) and are computed again using the pseudospectral methods. 
To obtain $\hat{\psi}_{k, j}^{n}$ at each new time level we solve $A \overrightarrow{\mathcal{U}}=\vec{b}$ where $A$ is a matrix of the form

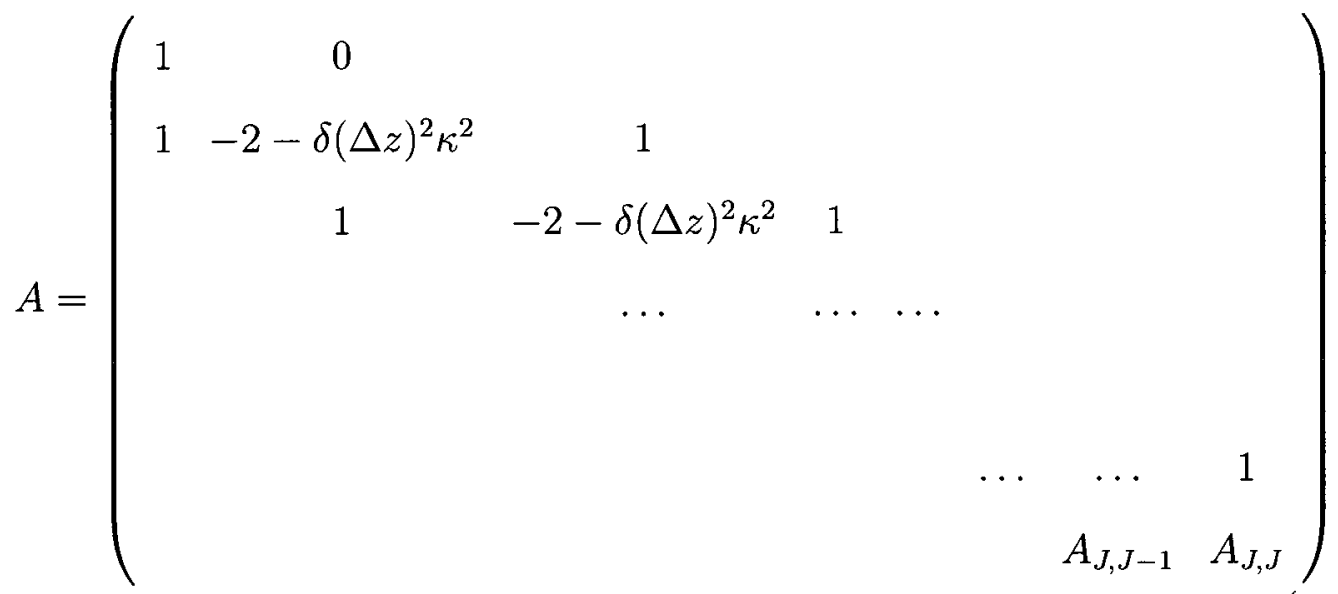

and $\vec{b}$ is a vector of the form

$$
\vec{b}=(\Delta z)^{2}\left(\begin{array}{c}
\hat{\zeta}_{k, 1}^{n} \\
\hat{\zeta}_{k, 2}^{n} \\
\hat{\zeta}_{k, 3}^{n} \\
\cdots \\
\\
\hat{\zeta}_{k, J-1}^{n} \\
b_{J}
\end{array}\right)
$$

In the standard run we used $\Delta z=0.1$ and $\Delta t=0.001$ for similar reasons as in the Rossby wave problem. The only difference is that in the gravity wave configuration the forced boundary $z_{1}$ is at the bottom and so $\Delta z$ is positive. As before the entries $A_{J, J}, A_{J, J-1}$ and $b_{J}$ are determined by the condition imposed at $z=z_{2}$.

For the zero boundary $A_{J, J}=1, A_{J, J-1}=0$ and $b_{J}=0$ so that $\hat{\psi}_{k, j}^{n}=0$. 
The steady boundary condition is given by equation (3.24) which is discretized using the backward finite difference approximation to obtain

$$
(1+\operatorname{sgn}(k) i|m| \Delta z) \hat{\psi}_{k, J}^{n}-\hat{\psi}_{k, J-1}^{n}=0
$$

This implies that $A_{J-1, J}=0, A_{J, J}=1+\operatorname{sgn}(k) i|m| \Delta z$ and $b_{J}=0$ where $m= \pm \sqrt{\frac{N^{2}}{(\bar{u}-c)^{2}}-\delta \kappa^{2}}$.

\subsection{Local in time implementation of the linear time-dependent radiation condition}

The convolution integrals in the time-dependent radiation conditions for Rossby and gravity waves are nonlocal in time. Computing these convolution integrals means that we need to sum up information from all previous levels. A less expensive method of computation is to make the discretization local in time. It consists of splitting the integral into a sum of 2 integrals.

The time-dependent radiation condition for Rossby waves is given by equation (3.38), we split it into two integrals to get

$$
\begin{aligned}
\frac{\partial}{\partial y} \hat{\psi}(\kappa, y, t)-\delta^{1 / 2} \kappa \hat{\psi}(\kappa, y, t) & =\int_{0}^{\bar{t}} \hat{\psi}(\kappa, y, \tau) \mathcal{H}(\kappa, t-\tau) d \tau \\
& +\int_{\bar{t}}^{t} \hat{\psi}(\kappa, y, \tau) \mathcal{H}(\kappa, t-\tau) d \tau
\end{aligned}
$$


and let $\bar{t}=t_{n-1}$ and $t=t_{n}$ so that the semi-discrete form of (4.34) is given by

$$
\left(1+\delta^{1 / 2} \kappa \Delta y\right) \hat{\psi}_{k, J}^{n}-\hat{\psi}_{k, J-1}^{n}=\Delta y C^{n}
$$

where

$$
\begin{array}{r}
C^{n}=\int_{0}^{t_{n-1}} \hat{\psi}(\kappa, y, \tau) \mathcal{H}\left(\kappa, t_{n}-\tau\right) d \tau+\int_{t_{n-1}}^{t_{n}} \hat{\psi}(\kappa, y, \tau) \mathcal{H}\left(\kappa, t_{n}-\tau\right) d \tau \\
\approx \int_{0}^{t_{n-1}} \hat{\psi}(\kappa, y, \tau) \mathcal{H}\left(\kappa, t_{n-1}-\tau\right) d \tau+\int_{t_{n-1}}^{t_{n}} \hat{\psi}(\kappa, y, \tau) \mathcal{H}\left(\kappa, t_{n}-\tau\right) d \tau \\
=C^{n-1}+\Delta C^{n}
\end{array}
$$

such that at each time level we just sum up the actual $\Delta C^{n}$ to the old $C^{n-1}$ to get $C^{n}$. Therefore for the Rossby wave time-dependent radiation condition we set $A_{J-1, J}=-1, A_{J, J}=1+\delta^{1 / 2} \kappa \Delta y$ and $b_{J}=\frac{C^{n}}{\Delta y}$.

At $t=0$ we have $C^{0}=0$. At the first time step $n=1, t=\Delta t$ and so $C^{1}=C^{0}+\Delta C^{1}$. We implement $\Delta C^{n}$ using the trapezoidal rule to yield

$$
\begin{array}{r}
\Delta C^{n} \approx \frac{\Delta t}{2}\left(\hat{\psi}_{k, J}^{n-1} \mathcal{H}\left(\kappa, t_{n}-t_{n-1}\right)+\hat{\psi}_{k, J}^{n} \mathcal{H}\left(\kappa, t_{n}-t_{n}\right)\right) \\
=\frac{\Delta t}{2}\left(\hat{\psi}_{k, J}^{n-1} \mathcal{H}(\kappa, \Delta t)+\hat{\psi}_{k, J}^{n} \mathcal{H}(\kappa, 0)\right) \\
=\hat{\psi}_{k, J}^{n-1} \frac{\beta}{2 \delta^{1 / 2}} \frac{\Delta t}{2} e^{i \kappa\left(1+\frac{\beta}{2 \delta \kappa^{2}}\right) \Delta t}\left[\mathcal{J}_{1}\left(\frac{\beta \Delta t}{2 \delta \kappa}\right)+i \mathcal{J}_{o}\left(\frac{\beta \Delta t}{2 \delta \kappa}\right)\right] \\
+i \frac{\beta}{2 \delta^{1 / 2}} \frac{\Delta t}{2} \hat{\psi}_{k, J}^{n}
\end{array}
$$

We compute $C^{n-1}$ just once for the first iteration and then keep adding on it $\Delta C^{n}$ at each time level instead of computing the full integral at each time $t=t_{n}$. This method makes the algorithm run fast, easy to implement and less expensive, 
and is called the local in time discretization approximation technique. Since the expression for $C^{n}$ given in (4.36) is approximate, a small error is introduced at each time step as a result of the difference between $\mathcal{H}\left(\kappa, t_{n}-\tau\right)$ and $\mathcal{H}\left(\kappa, t_{n-1}-\tau\right)$. The order of magnitude of the error that will accumulate from $t=0$ to $t=t_{n}$ is $O\left(\Delta t \Delta y t_{n-1}\right)$. With our choice of $\Delta t=10^{-3}$ and $\Delta y=10^{-1}$ the error is $\sim 10^{-2}$ by $t=100$.

For the gravity wave problem we compute $C^{n} \approx C^{n-1}+\Delta C^{n}$ where $\Delta C^{n}$ is given by

$$
\begin{array}{r}
\Delta C^{n} \approx \frac{\Delta t}{2}\left(\hat{\psi}_{k, J}^{n-1} \mathcal{H}\left(\kappa, t_{n}-t_{n-1}\right)+\hat{\psi}_{k, J}^{n} \mathcal{H}\left(\kappa, t_{n}-t_{n}\right)\right) \\
=-\delta^{1 / 2} \kappa \frac{\Delta t}{2}\left(\hat{\psi}_{k, J}^{n-1} g(\Delta t) e^{-i \kappa \bar{u}_{J} \Delta t}+\hat{\psi}_{k, J}^{n} g(0)\right) \\
=-\delta^{1 / 2} \kappa \frac{\Delta t}{2} \hat{\psi}_{k, J}^{n-1} g(\Delta t) e^{-i \kappa \bar{u}_{J} \Delta t}
\end{array}
$$

and $g(\Delta t)$ is a real number and is given by

$$
g(\Delta t)=\int_{0}^{\Delta t} \frac{a}{\sigma} \mathcal{J}_{1}(a \sigma) d \sigma
$$

and $\mathcal{J}_{1}(a \sigma)$ is the Bessel function of first kind of order 1. So the integrand in equation (4.39) can be approximated (Abramowitz and Stegun 1964) by the first few terms to get

$$
\frac{a}{\sigma} \mathcal{J}_{1}(a \sigma)=\frac{a^{2}}{2}\left[1-\frac{a^{2} \sigma^{2}}{8}+\frac{a^{4} \sigma^{4}}{192}+\ldots \ldots \ldots\right]
$$

for $\sigma<<1$ so that $g(\Delta t)$ is given by 


$$
\begin{array}{r}
g(\Delta t)=\int_{0}^{\Delta t} \frac{a}{\sigma} \mathcal{J}_{1}(a \sigma) d \sigma=\int_{0}^{\Delta t} \frac{a^{2}}{2}\left[1-\frac{a^{2} \sigma^{2}}{8}+\frac{a^{4} \sigma^{4}}{192}+\ldots \ldots \ldots\right] d \sigma \\
=\frac{a^{2}}{2}\left[1-\frac{a^{2}}{24} \Delta t^{3}+\frac{a^{4}}{960} \Delta t^{5}+O\left(\Delta t^{7}\right)\right]
\end{array}
$$

So for the gravity wave time-dependent radiation condition we set $A_{J, J}=1+$ $\delta^{1 / 2} \kappa \Delta z, A_{J, J-1}=-1$ and $b_{J}=\frac{C^{n}}{\Delta z}$.

For the case $\delta \neq 0$ the expression for $C^{n}$ is approximate as in the Rossby wave case. So an error of magnitude $\sim 10^{-2}$ would accumulate by $t=100$. However for the case $\delta=0$ the relation $C^{n}=C^{n-1}+\Delta C^{n}$ is exact because the integrand in (3.56) is independent of $t$.

\subsection{Implementation of the nonlinear correction}

In section 3.3 we derived an expression for a nonlinear correction that can be added to the time-dependent linear radiation condition. The nonlinear correction comprises a contribution for the wavenumber $\kappa=0$ only and it is a nonlocal in time integral of the $y$ derivative of the momentum flux for Rossby waves and of the $z$ derivative of the momentum flux for gravity waves. The $y$ derivative of the momentum flux is approximated by

$$
\frac{\partial F}{\partial y} \approx \frac{F_{j}^{n}-F_{j-1}^{n}}{\Delta y}
$$


where the momentum flux $F$ is given in the appendix (B) and can be approximated by

$$
F(y, t)=\frac{k_{o}}{2} \sum_{k=-\infty}^{+\infty} k \hat{\psi}_{k}^{*}(y, t) \frac{\partial}{\partial y} \hat{\psi}_{k}(y, t) \approx \frac{k_{o}}{2} \sum_{k=-K / 2}^{K / 2} k \hat{\psi}_{k, j}^{* n} \frac{\hat{\psi}_{k, j}^{n}-\hat{\psi}_{k, j-1}^{n}}{\Delta y}
$$

We use the local in time computation to approximate the nonlinear correction to obtain

$$
\begin{array}{r}
C_{0}^{n}=\varepsilon \int_{0}^{t_{n}} \frac{\partial}{\partial y} F(y, \tau) d \tau=\varepsilon \int_{0}^{t_{n-1}} \frac{\partial}{\partial y} F(y, \tau) d \tau+\varepsilon \int_{t_{n-1}}^{t_{n}} \frac{\partial}{\partial y} F(y, \tau) d \tau \\
=C_{0}^{n-1}+\Delta C_{0}^{n}
\end{array}
$$

and then add $C_{0}^{n}$ to the linear time-dependent radiation condition so that $b_{J}=$ $\frac{C^{n}+C_{0}^{n}}{\Delta y} \cdot C_{0}^{0}=0$ and $\Delta C_{0}^{n}$ is approximated using the trapezoidal rule. 


\section{Chapter 5}

\section{Results of the numerical}

\section{simulations}

For both Rossby and gravity waves, numerical simulations are carried out using configurations in which there is no critical layer and configurations in which there is critical layer. In section 5.1 we describe the Rossby wave simulations results while in section 5.2 we describe the results obtained from the simulations of gravity wave propagation. Each section in this chapter comprises three parts, the first part describes the results for the simulations carried out in configurations in which there is a critical layer in the flow and these results are used as test cases. This means that we first use the results from these simulations to verify the correctness of the numerical implementations by comparing with the results obtained by Campbell (2004) in the Rossby wave case and Campbell and Maslowe (2003) in gravity wave case.

The actual work for this project described in the remaining parts of each of the two sections deals with simulations carried out using configurations in which there is no critical layer and the waves propagate from the source level all the 
way to the opposite boundary where different boundary conditions described in Chapter 3 are implemented. The results are shown as graphs, we then compare these results and conclude that the time-dependent results give the most realistic results.

\subsection{Results of the simulations of the Rossby propagation}

\subsubsection{Results of the simulations in a configuration with a critical layer in the flow}

We solve the initial boundary value problem (4.1)-(4.4) using the numerical methods described in Chapter 4. Simulations are carried out on a rectangular domain given by $0 \leq x \leq 2 \pi$ and $-5 \leq y \leq 5$ with the following parameters; $\varepsilon=0.05, \beta=1.0$, and $\delta=0.2$ and $k_{o}=2$. The background flow was set to $\bar{u}=\tanh y$ so that the critical layer is located at $y_{c}=0$ as shown in Figure 5.1. In the nonlinear simulations a small amount of viscosity corresponding to the kinematic viscosity of $\nu=5 \cdot 10^{-5}$ was necessary to prevent the accumulation of wave activity at the high end of the wavenumber spectrum and near the forced boundary. The mean flow profile $\bar{u}=\tanh y$ does not satisfy the governing equation (4.1) with the viscous term added we need to add an extra term $\left(-\varepsilon^{-1} \nu \bar{u}^{\prime \prime \prime}\right)$ to the left hand side of (4.1). The wave source is at the upper boundary of the rectangular domain $y_{1}=5$ and has the form

$$
\psi\left(x, y_{1}, t\right)=2 \cos k_{o} x=e^{i k_{o} x}+c . c .
$$




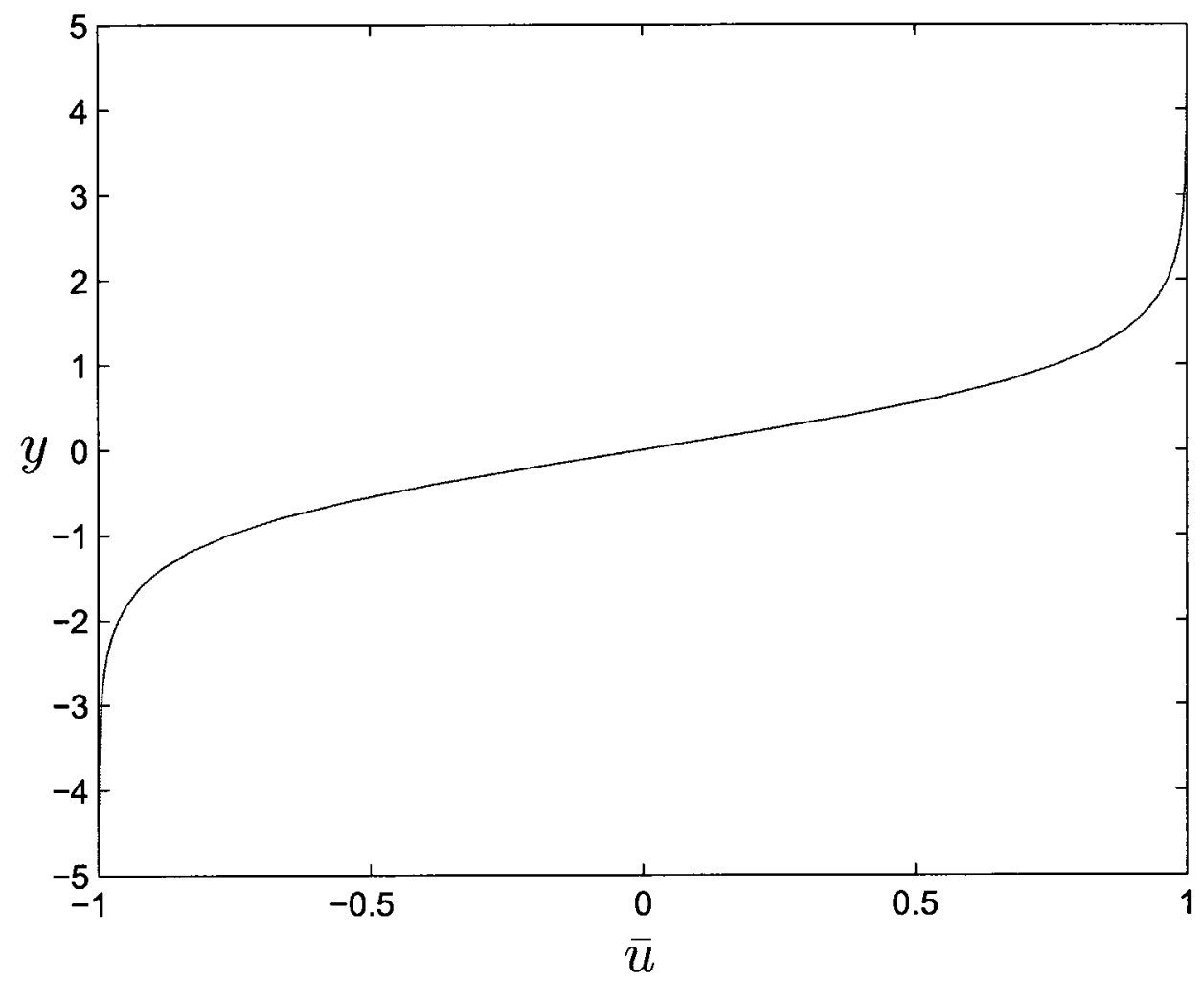

Figure 5.1: Simulation of Rossby wave propagation: the background flow speed is $\bar{u}=\tanh y$ as a function of the latitude $y$. The critical level is at $y_{c}=0$.

Simulations for both linear and nonlinear problems were carried out over the nondimensional time interval from $t=0$ to $t=70$ using the zero boundary condition and the results are presented in Figures 5.2, 5.3 and 5.4. These results are similar to those obtained by Campbell (2004), the only difference being that we have used $\varepsilon=0.05$ instead of $\varepsilon=0.02$. The advantage of using a large value of $\varepsilon$ is that phenomena from nonlinear interactions are observed at earlier time preventing us from having to run the program for very large time.

As is shown in Figure 5.2(a) corresponding to linear simulation and Figure 
$5.2(b)$ to nonlinear simulation, there is little or no transmission of wave activity beyond the critical level. wave absorption and reflection can be observed by examining the behavior of the meridional momentum flux $F$ in the vicinity of the critical layer. At each time step, the jump in the momentum flux $[F]$ across the critical level was computed and the corresponding results are presented in Figure 5.3. The divergence of the momentum flux $\frac{\partial F}{\partial y}$ results in a change in the mean flow according to $\frac{\partial \bar{u}_{0}}{\partial t}=-\varepsilon^{2} \frac{\partial F}{\partial y}$ described in appendix B. So when $[F]$ is positive the waves are absorbed and the mean flow accelerates. The dashed line corresponds to the linear simulation, at about $t=20$ this line is almost constant indicating that there is continuous absorption of the wave by the mean flow. The nonlinear simulation result corresponds to the solid line, the jump $[F]$ drops to zero and to negative values indicating first reflection and then start to oscillate from positive values indicating absorption to negative values indicating over-reflection and at the same time decays as $t$ becomes large.

The variation of the momentum flux $F$ at time $t=70$ as a function of latitude $y$ is shown in Figure 5.4 for both linear (dashed line) and nonlinear (solid line) simulations the momentum flux is positive above the critical level and suddenly drops to zero below the critical level. This is in agreement with the Eliassen-Palm theorem in Appendix B which states that the momentum flux is independent of latitude $y$ in the absence of singularities (critical level). From these observations we conclude that we do not need to implement a radiation condition at the outflow boundary if there is a critical level in the flow and so the zero boundary condition is enough. 

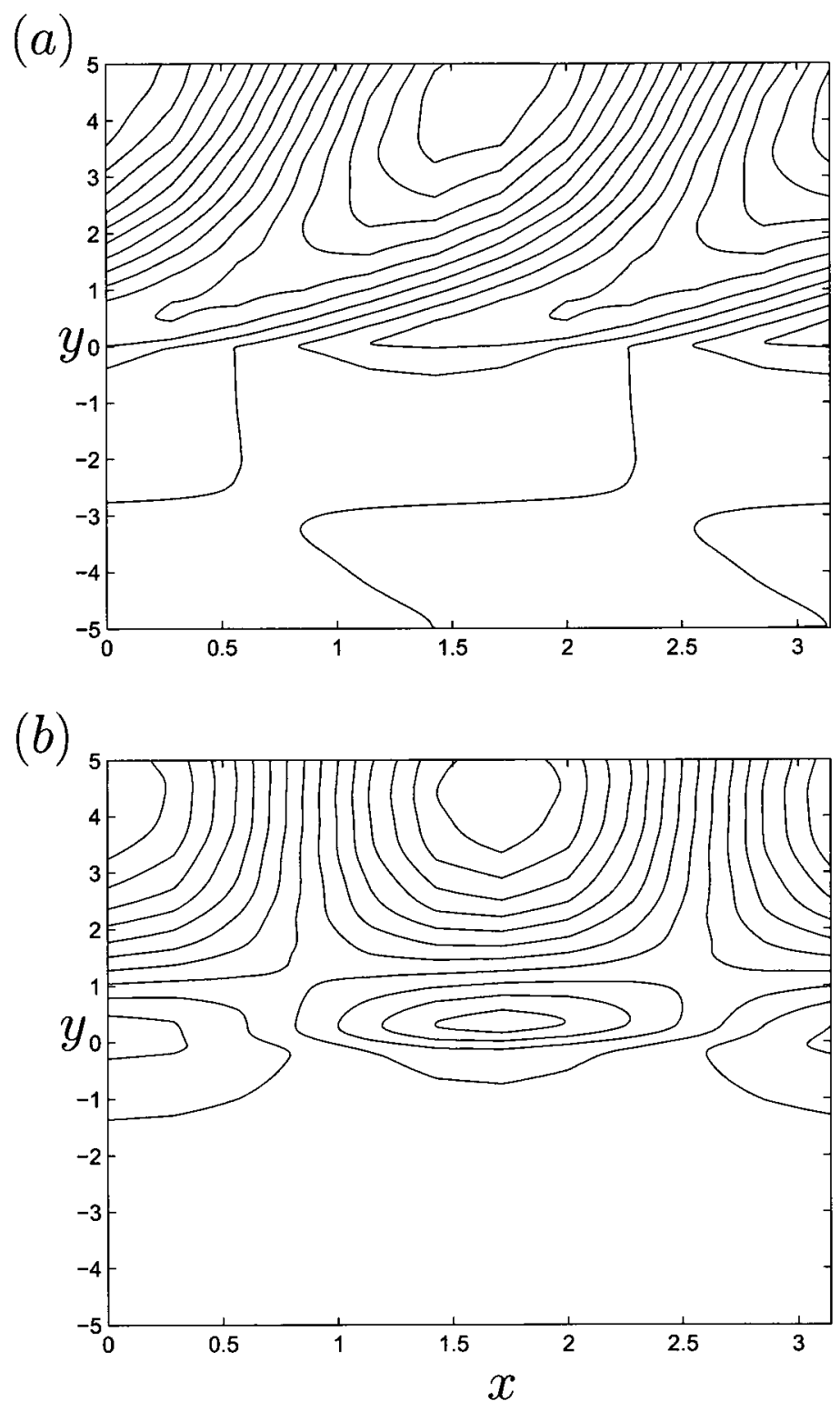

Figure 5.2: Linear and nonlinear simulations of Rossby wave propagation: contour plot of $\Psi(x, y, t)$ at $t=70,(a)$ linear simulation with $\epsilon=0$ and $(b)$ nonlinear simulation with $\epsilon=0.05$. In both $(a)$ and $(b) \delta=0.2$ and $\beta=1$. A periodic forcing $e^{i k_{o} x}, k_{o}=2$, has been applied at the upper boundary of the rectangular domain. The background flow speed is $\bar{u}=\tanh y$, the critical level is at $y=0$. Both the contour show that there are not much transmission activity beyond the critical layer. 


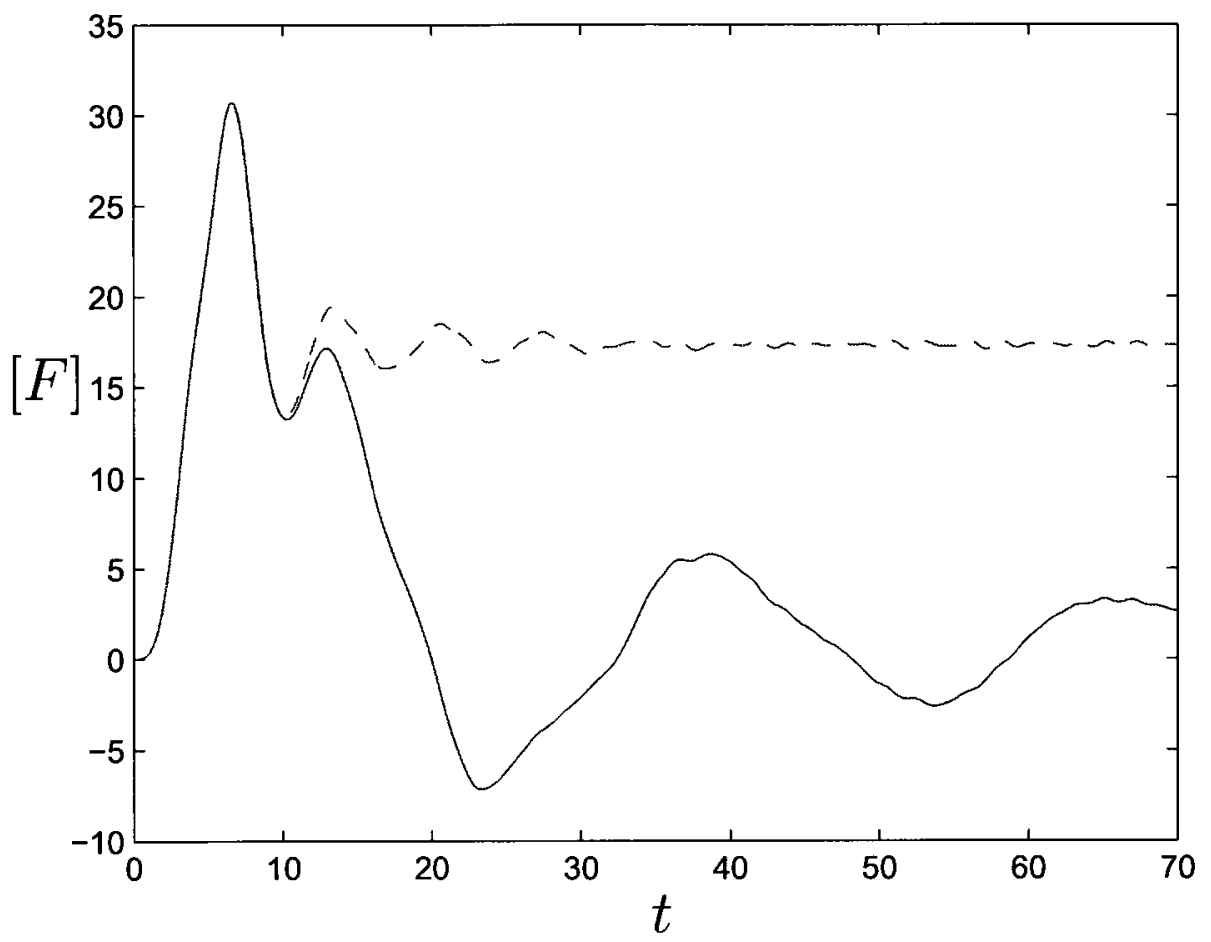

Figure 5.3: Linear and nonlinear simulations of Rossby wave propagation: plot of the jump in the momentum flux $[F]$ across the critical layer as a function of time for $0 \leq t \leq 70$. A periodic forcing $e^{i k_{o} x}, k_{o}=2$, has been applied at the upper boundary of the rectangular domain, $\delta=0.2$ and $\beta=1$. The background flow speed is $\bar{u}=\tanh y$, the critical level is at $y=0$. The dashed line shows the result obtained for the linear simulation $(\varepsilon=0)$ while the solid line shows the result obtained for the nonlinear simulation $(\varepsilon=0.05)$. In the linear case $[F]$ becomes almost constant by about $t=20$. This indicates absorption of the waves by the mean flow. In the nonlinear case $[F]$ alternates between positive values (absorption) and the negative values (overreflection). 


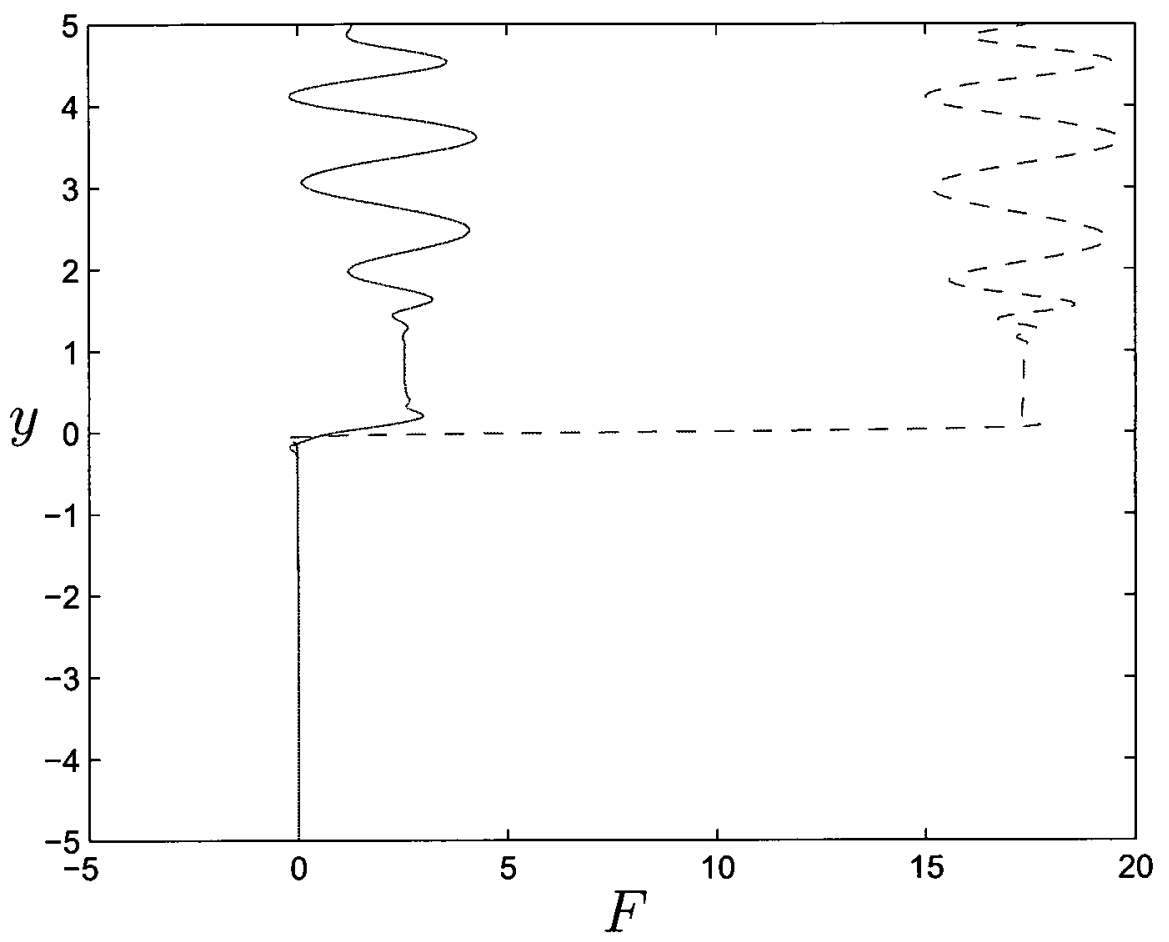

Figure 5.4: Linear and nonlinear simulations of Rossby wave propagation: plot of the momentum flux as function of the latitude $y$ at the time $t=70$. A periodic forcing $e^{i k_{o} x}, k_{o}=2$, has been applied at the upper boundary of the rectangular domain, $\delta=0.2$ and $\beta=1$. The background flow speed is $\bar{u}=\tanh y$, the critical level is at $y=0$. The dashed line corresponds to the linear simulation $(\varepsilon=0)$ while the solid line corresponds to the nonlinear simulation $(\varepsilon=0.05)$.

\subsubsection{Results of simulations of Rossby wave propagations in a configuration without a critical layer in flow}

In this section we show the results of the simulations carried out using a configuration in which there is no critical layer. The simulations were carried out with the same parameters and the same forcing as in the configuration in which there is a critical layer, the only difference being that the background flow was 
modified in order to remove the critical level. In the absence of a critical layer the waves propagate all the way to the outflow boundary. So it is important to correctly implement the boundary condition at the outflow boundary. The results shown here are for the mean flow profile $\bar{u}=1$.

Three types of boundary conditions are implemented at the outflow boundary; the zero boundary condition, the steady radiation condition and the timedependent radiation condition. The results obtained from linear simulations of Rossby wave propagation are shown in Figures 5.5-5.9. The results from the linear simulations are almost the same for $t \leq 10$, some differences are clearly observed for $t>10$. In these simulations $k_{o}=2, \beta=1, \delta=0.2$ and $\bar{u}=1$, so the meridional wavenumber is $l=\sqrt{\frac{\beta}{\bar{u}}-\delta k_{o}^{2}} \approx 0.45$ and we except to have a meridional wavelength of about $2 \pi / l \approx 14$. The magnitude of $l$ relative to $k_{o}$ determines the slant of the contour. The analytical solution given in section 2.5 tells us the solution comprises a steady term periodic in the $y$-direction with meridional wavelength $2 \pi / l$ where $l^{2}=\frac{\beta}{\bar{u}}-\delta k_{o}^{2}$, as well as a transient term.

The results obtained with a zero boundary condition are shown in Figure 5.5. At $t=23$ the waves have reached the lower boundary and are forced to stay inside the rectangular domain. By $t=45$ and $t=62$ it appears that there is superposition of the incident wave with wavenumber $l$ and a reflected wave with a different wavenumber and wavelength.

Figure 5.6 shows the results obtained with the steady radiation condition. In this case there is wave transmission at the outflow boundary. However one can see from Figure 5.6 that the slant of the contours and the direction of propagation of the wave is fixed near the outflow boundary. This is because the steady radiation condition fixes the meridional wavenumber $l \approx 0.45$ and does 
not not take into account the transient part of the solution. Again there appears to be wave reflection that cause deformations of the contours observed for $t>10$ in Figure 5.6, as well as the variation of the wavelength and the increase in the amplitude of the streamfunction at large time as seen in Figure 5.8.

If we look at Figure 5.7, we see that for $t>23$ there are no deformations of the contours, the meridional wavelength does not change much with time and waves evolve with time near the outflow boundary. This can be explained by the fact that the time-dependent radiation condition takes into account the transient evolution of the wave. The momentum flux for this simulations is shown by the solid curve in Figure 5.9. We see that the momentum flux is approximately constant and independent of the latitude $y$ which is agreement with the Eliassen-Palm theorem which states that for a linear wave the momentum flux is independent of $y$ in the absence of any singularity (critical level). In contrast the momentum flux profiles for the zero and steady boundary condition simulations vary significantly with $y$. We conclude that the time-dependent radiation condition gives the better results than the other two boundary conditions.

The contour plots shown in Figure 5.10 are the results of the linear simulations in the long-wave limit $\delta=0$. For $t \leq 10$ the contour plots obtained with the time-dependent radiation condition with $\delta=0$ are similar to those shown in Figure 5.7 obtained with $\delta \neq 0$. As seen in Figure 5.10(e) at a later time $(t \gtrsim 45)$ the solution reaches a steady state and is almost exactly periodic in the $y$-direction as predicted by the exact solution (2.103). 
(a) $t=2$

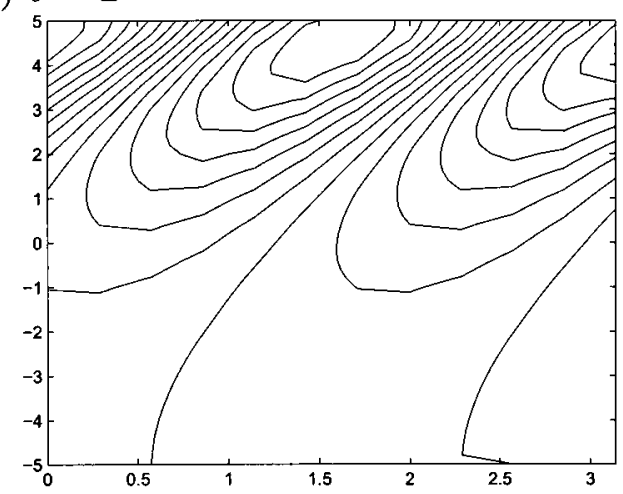

(c) $t=10$

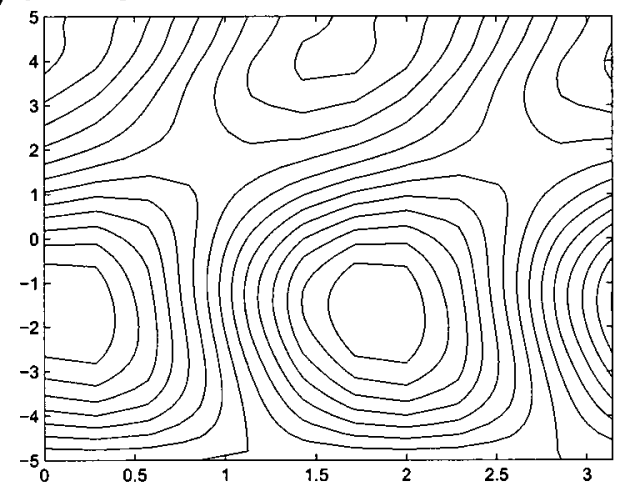

(e) $t=42$

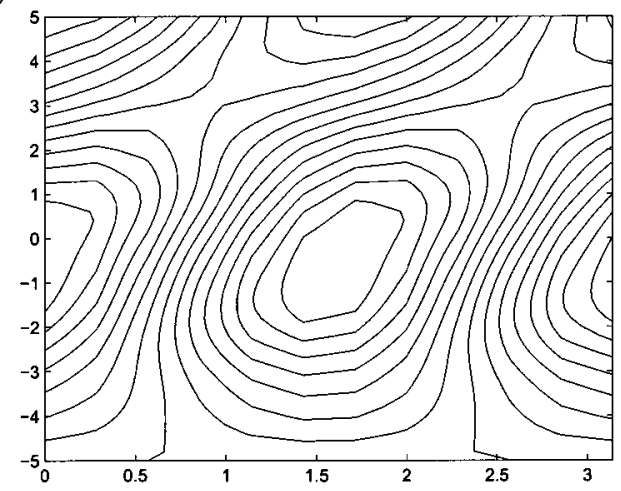

(b) $t=5$

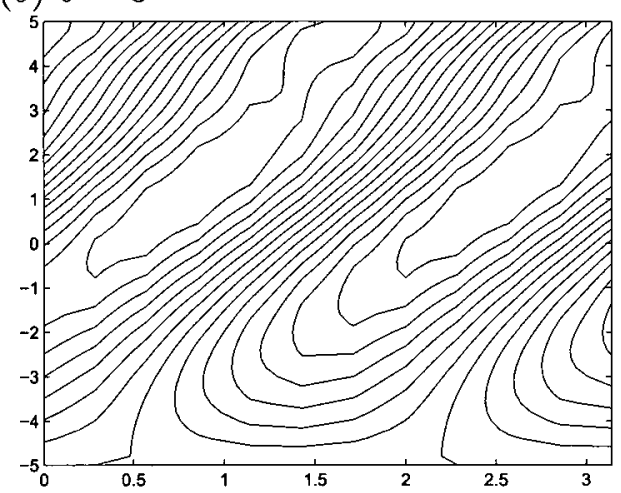

(d) $t=23$

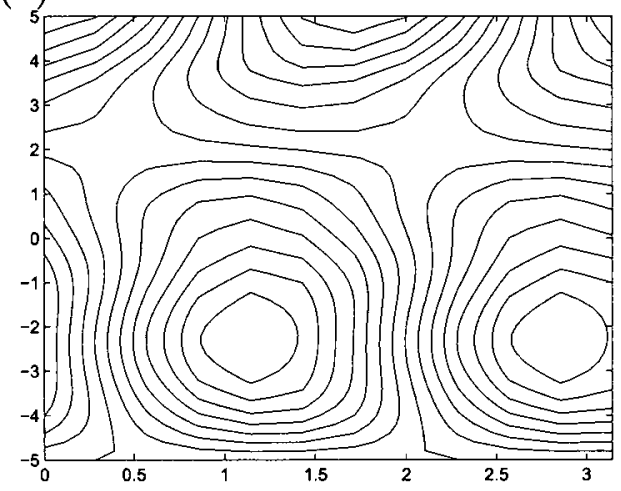

(f) $t=62$

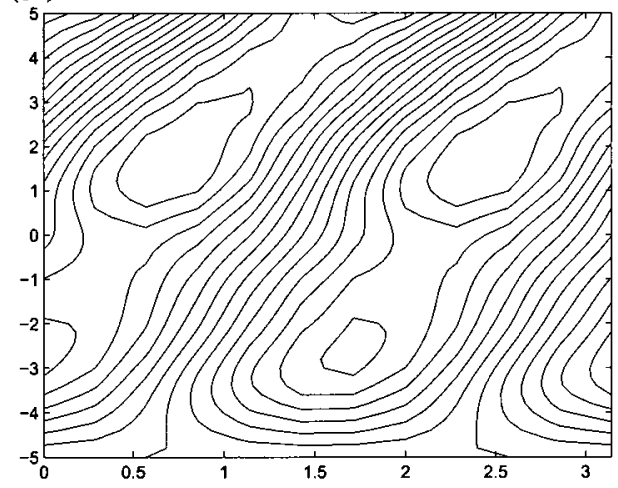

Figure 5.5: Linear simulation of Rossby wave propagation $(\varepsilon=0)$ with the zero boundary condition: contour plots of the streamfunction $\psi(x, y, t)$ obtained at (a) $t=2$, (b) $t=5$, (c) $t=10$, (d) $t=23$, (e) $t=42$ and (f) $t=62$. A periodic forcing $e^{i k_{o} x}, k_{o}=2$, has been applied at the upper boundary of the rectangular domain, $\delta=0.2$ and $\beta=1$. The background flow is constant, $\bar{u}=1$. 
(a) $t=2$

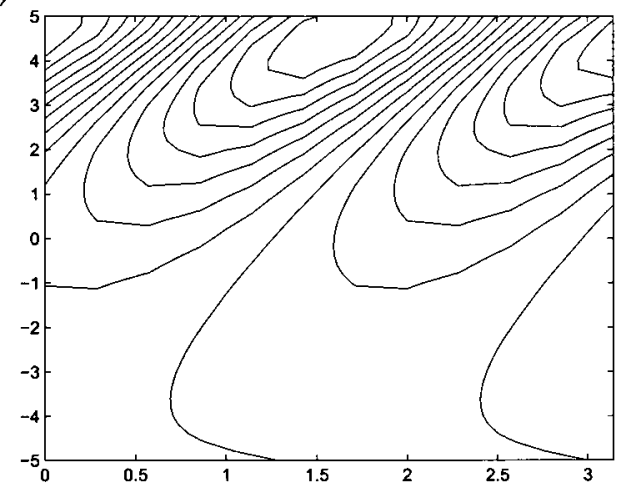

(c) $t=10$

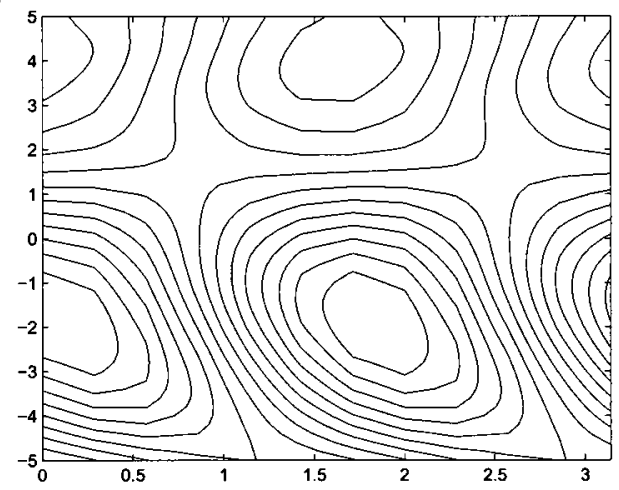

(e) $t=42$

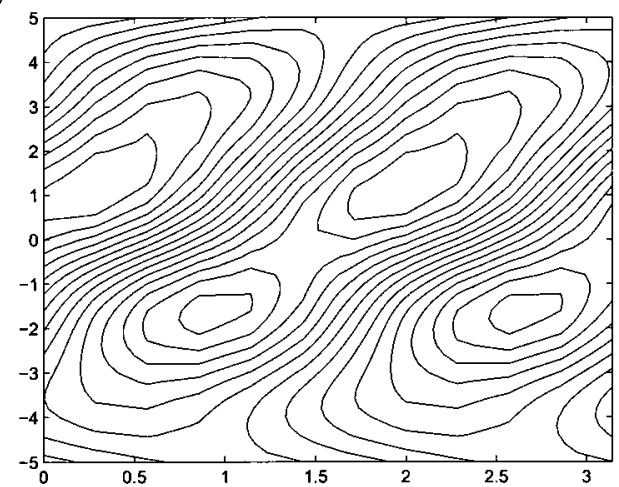

(b) $t=5$

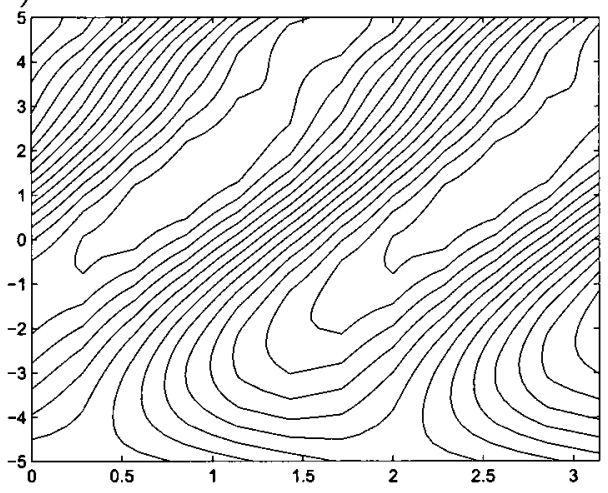

(d) $t=23$

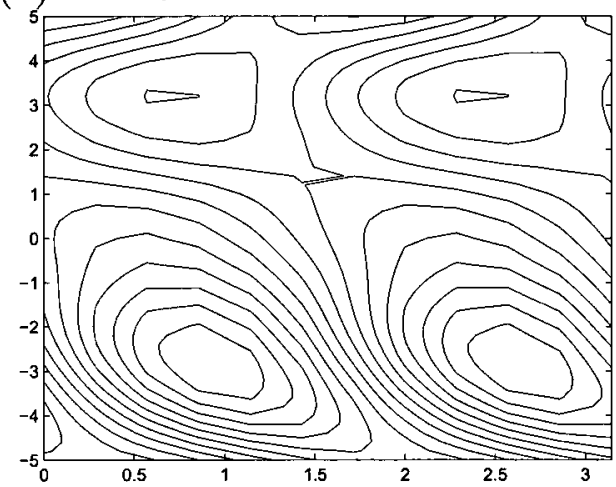

(f) $t=62$

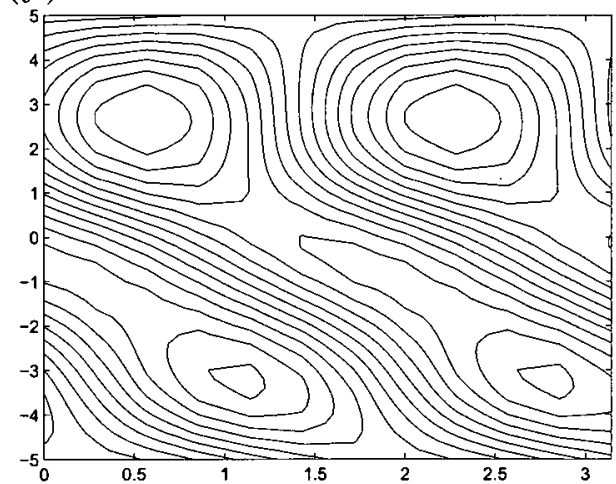

Figure 5.6: Linear simulation of Rossby wave propagation $(\varepsilon=0)$ with the steady boundary condition: contour plots of the streamfunction $\psi(x, y, t)$ obtained at (a) $t=2$, (b) $t=5$, (c) $t=10$, (d) $t=23$, (e) $t=42$ and (f) $t=62$. A periodic forcing $e^{i k_{o} x}, k_{o}=2$, has been applied at the upper boundary of the rectangular domain, $\delta=0.2$ and $\beta=1$. The background flow is constant, $\bar{u}=1$. 
(a) $t=2$

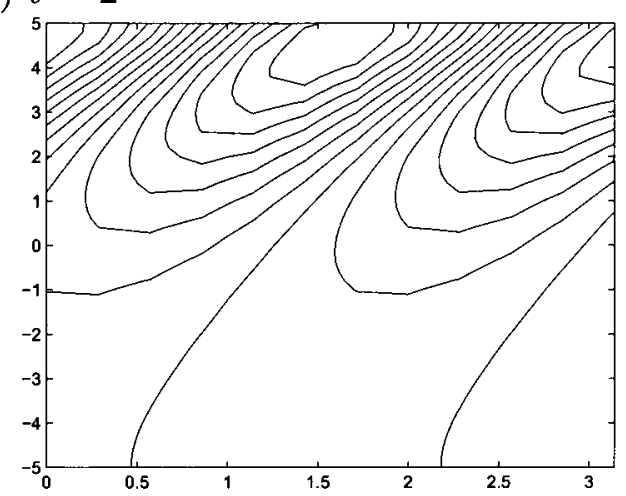

(c) $t=10$

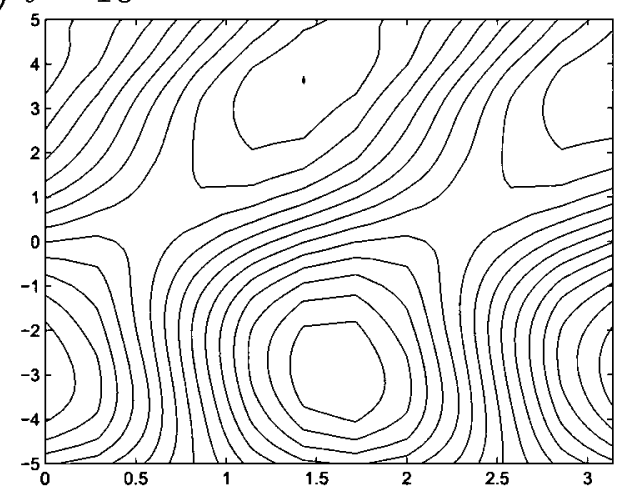

(e) $t=42$

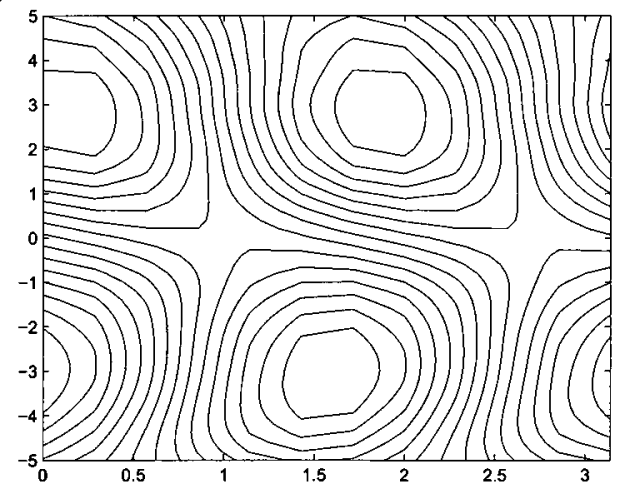

(b) $t=5$

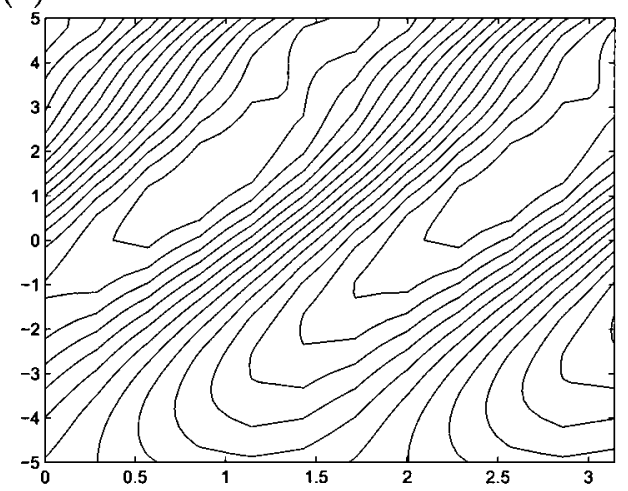

(d) $t=23$

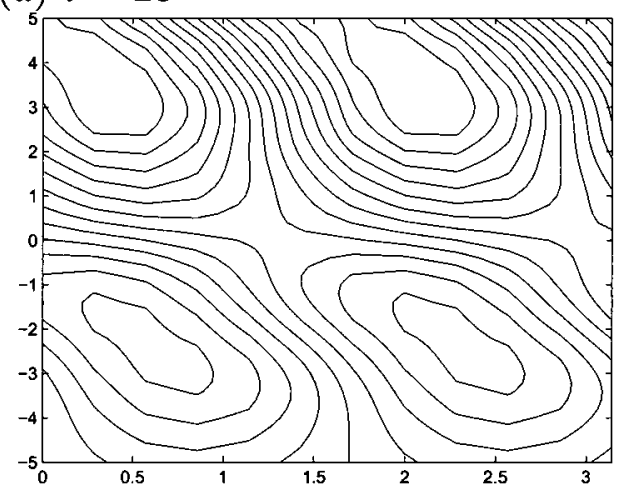

(f) $t=62$

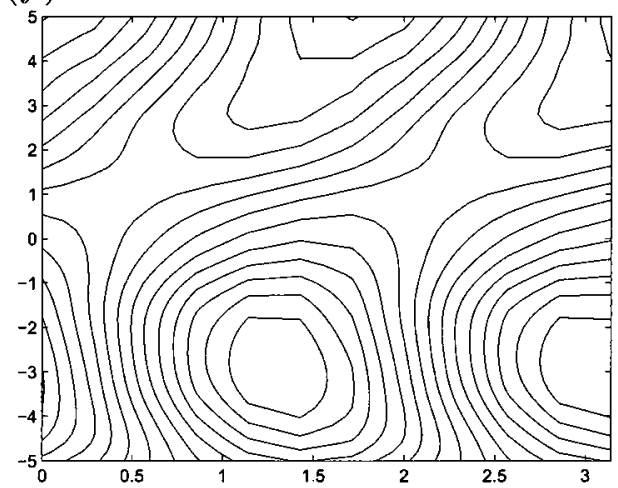

Figure 5.7: Linear simulation of Rossby wave propagation $(\varepsilon=0)$ with the timedependent radiation condition: contour plots of the streamfunction $\psi(x, y, t)$ obtained at (a) $t=2$, (b) $t=5$, (c) $t=10$, (d) $t=23$, (e) $t=42$ and (f) $t=62$. A periodic forcing $e^{i k_{o} x}, k_{o}=2$, has been applied at the upper boundary of the rectangular domain, $\delta=0.2$ and $\beta=1$. The background flow is constant, $\bar{u}=1$. 


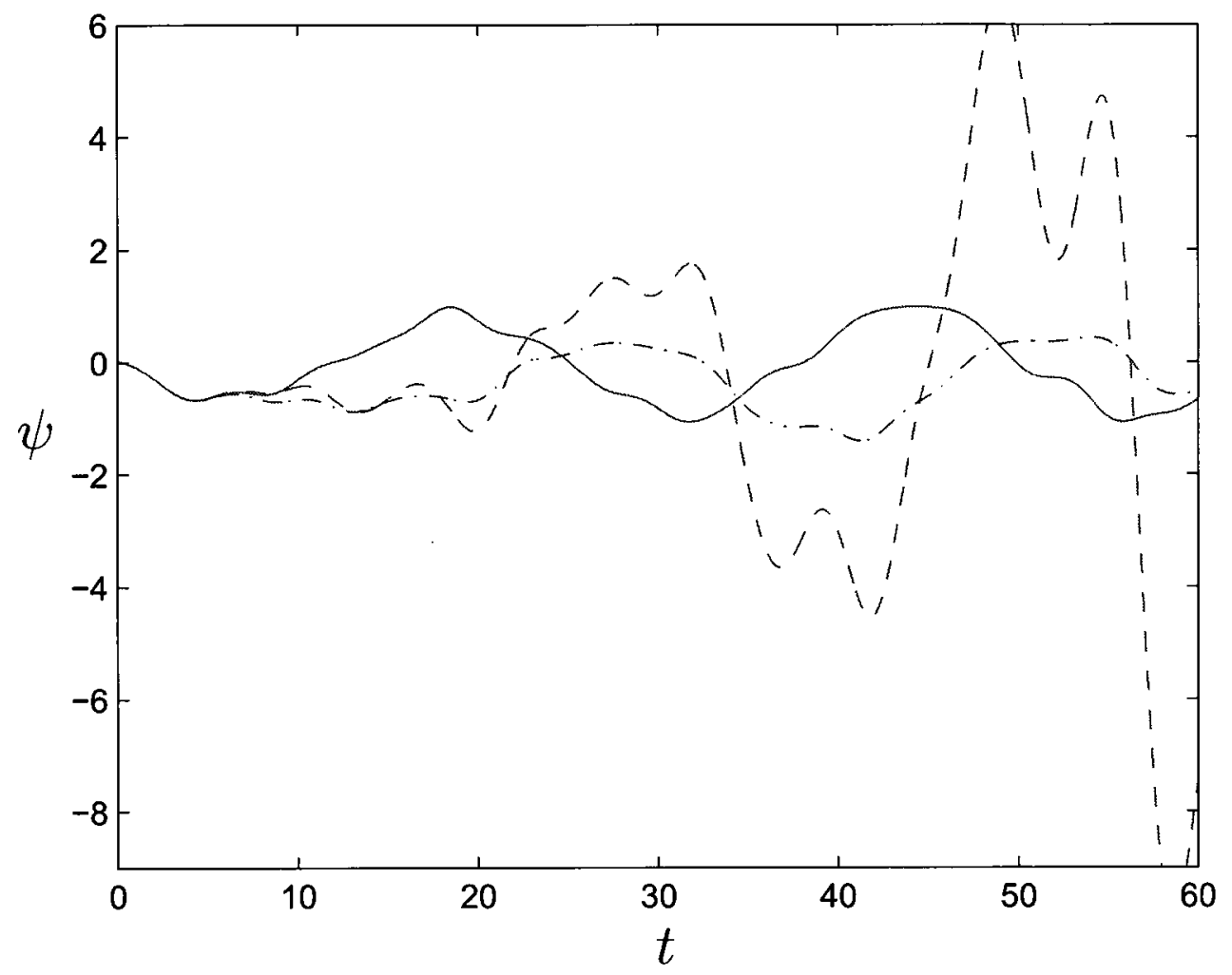

Figure 5.8: Linear simulation of Rossby wave propagation: plot of the streamfunction $\psi(x, y, t)$ as a function of time $\mathrm{t}$ at $x=0.6$ and $y=-1,0 \leq t \leq 60$. A periodic forcing $e^{i k_{o} x}, k_{o}=2$, has been applied at the upper boundary of the rectangular domain, $\delta=0.2$ and $\beta=1$. The background flow is constant, $\bar{u}=1$. The solid line corresponds to the simulation with time-dependent radiation condition and the dashed-dotted line corresponds to the simulation with the zero boundary condition whereas the dashed line is the result obtained for the simulation with the steady radiation condition. 


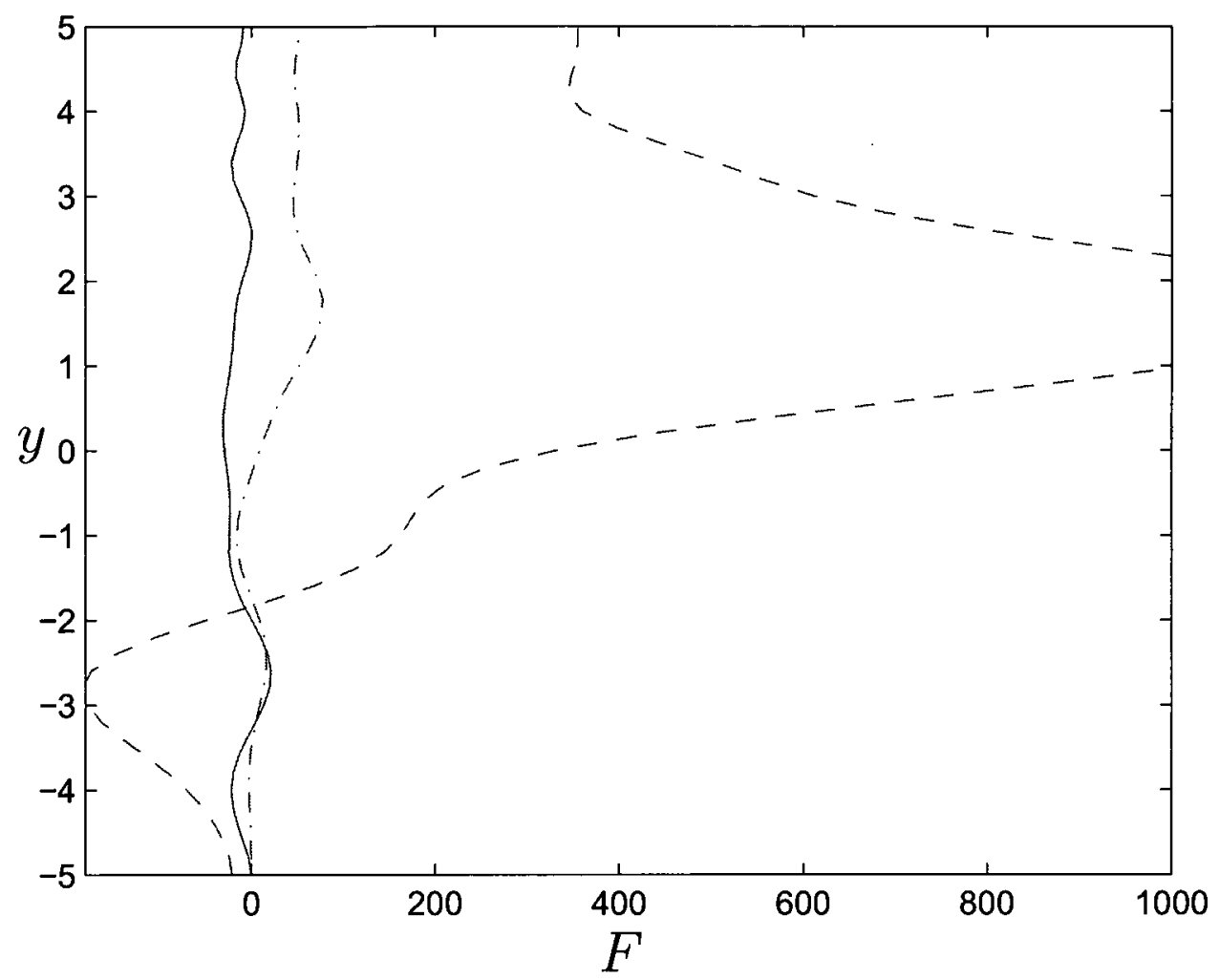

Figure 5.9: Linear simulation of Rossby wave propagation: plot of the averaged horizontal momentum flux $F$ as a function of $y$ at the time $t=40$. A periodic forcing $e^{i k_{o} x}, k_{o}=2$, has been applied at the upper boundary of the rectangular domain, $\delta=0.2$ and $\beta=1$. The background flow is constant, $\bar{u}=1$. The solid line corresponds to the simulation with time-dependent radiation condition and the dashed-dotted line corresponds to the simulation with the zero boundary condition whereas the dashed line is the result obtained for the simulation with the steady radiation condition. 


\subsubsection{The results of nonlinear simulations}

The results from the nonlinear simulations $(\varepsilon=0.05)$ of the Rossby waves propagation are shown in Figures 5.11-5.14. In order to see the effect of nonlinearity, simulations were carried out up to $t=85$. Figure 5.11 shows the results obtained with the zero boundary condition, the downward propagation of waves is observed if one compares the contour plots of the streamfunction $\psi(x, y, t)$ at $t=80$ and $t=85$, however the waves are prevented from propagating further because of the zero boundary condition. This is clearly shown in the contour plots of the streamfunction at $t=23$ and $t=85$. The same conclusion as for linear simulations can be drawn also with the exception being that there are interactions between different harmonics (zonal wavenumbers) as a result of nonlinearities, and there is an increase in the overall wave amplitude at large $t$.

The results from nonlinear simulations are presented in Figure (5.12) for the case where the steady radiation condition is implemented at the outflow boundary. As the simulations are continued to late time, the contour plots deform as a result of nonlinear interactions and the solution breaks down. This can be observed by comparing the contour plots in Figures 5.12(d), 5.12(e) and $5.12(\mathrm{f})$ at the times $t=62, t=67$ and $t=69$ respectively. As one can see in Figure 5.12(f) instabilities near the outflow boundary start to develop at $t=69$ and the nonlinear simulations fail at about $t=73$.

Figure 5.13 shows the results obtained with the time-dependent radiation condition. If the time-dependent radiation condition is applied at the outflow boundary, the simulations are able to continue to late time without the development of instabilities. Figure 5.14 shows the wave amplitude at fixed $y$ as a function of time for each of the three boundary conditions. The steady bound- 
ary condition leads to increased wave amplitude due to the instabilities seen in Figure 5.12(f). We conclude from Figures 5.11-5.14 that the time-dependent radiation condition is the most stable.

By applying the time-dependent radiation condition we take into account the transient evolution of the waves, we are able to avoid the instabilities and wave reflections and increased amplitude that occur with the implementation of the zero and the steady boundary conditions.

As a benchmark of for comparing the results obtained with the different boundary conditions we carry out an additional simulation using the same forced boundary condition at $y=5$ and the same input parameters but in an extended domain $-25<y<5$ with the outflow boundary at $y=-25$. By extending the domain we can obtain more accurate results within the time frame that it takes the waves to reach the outflow boundary $y=-25$, i.e up until about $t=15$.

In Figure 5.15 we compare the contour plots of the streamfunction $\psi$ obtained using the zero boundary condition, the steady radiation condition and the timedependent radiation condition in the domain $-5<y<5$ with that obtained using a zero boundary condition in the extended domain $-25<y<5$ at the time $t=5$. At this time the waves have not reached the level $y=-25$ so it is reasonable to assume that the simulation on the extended domain is the most accurate. Of the 3 simulations on the domain $-5<y<5$ the one with the time-dependent boundary condition shows the closest agreement with that on the extended domain, both with regard to the shape of the contours near the outflow boundary $y=-5$ and to the contour levels.

Figures 5.15 and 5.17 also support this conclusion. We plot the wave amplitude and the zonally averaged momentum flux at a fixed value of the latitude 
$y=-1$ for each of the configuration shown in Figure 5.15. In each figure the solid curve corresponds to the configuration with the extended domain $-25<y<5$ which can be assumed to be the most accurate at least at early time before the waves reach the outflow boundary. In each case the curve corresponding to time-dependent radiation condition is the closest. 
(a) $t=2$

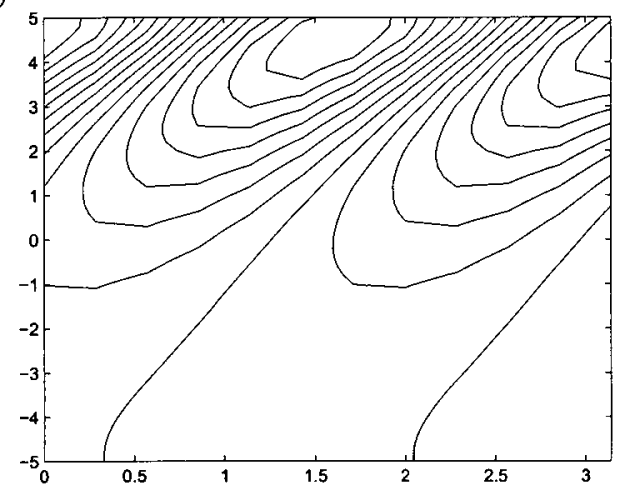

(c) $t=10$

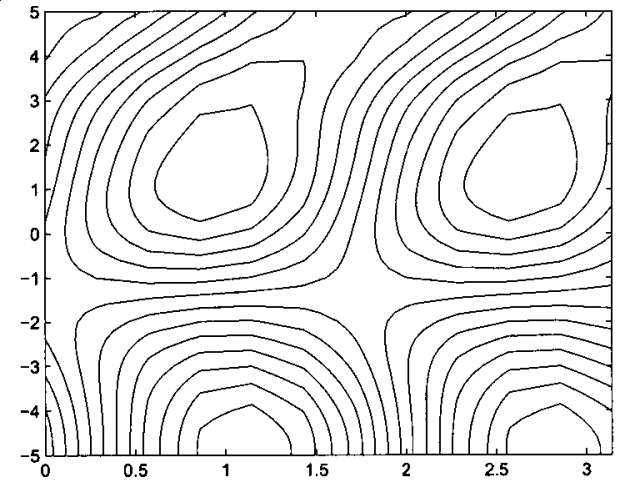

(e) $t=45$

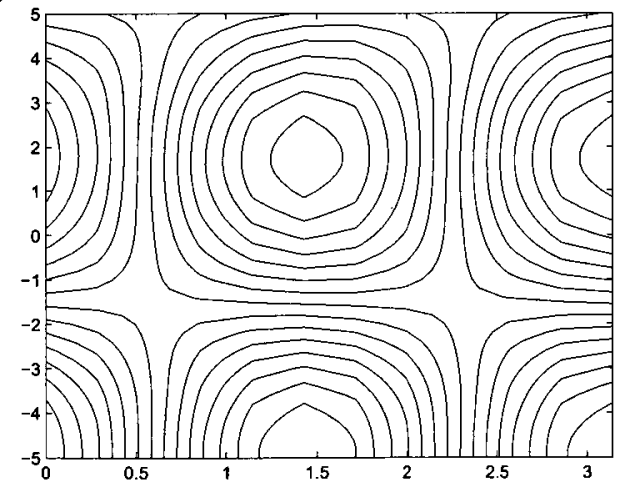

(b) $t=5$

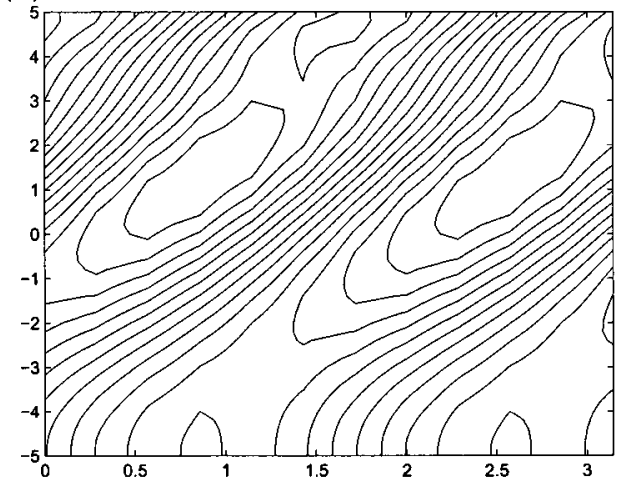

(d) $t=23$

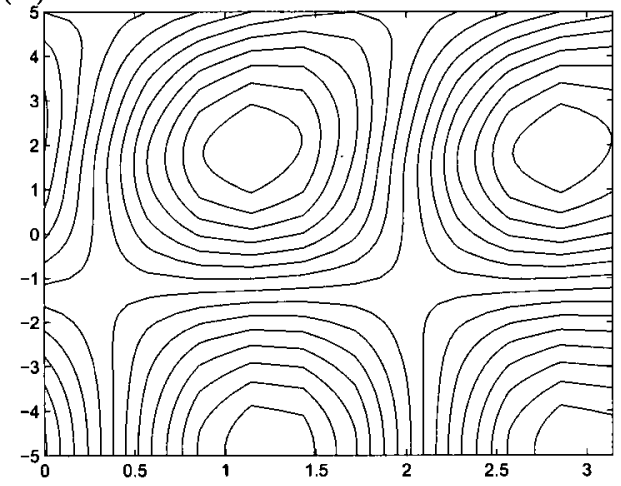

(f) $t=62$

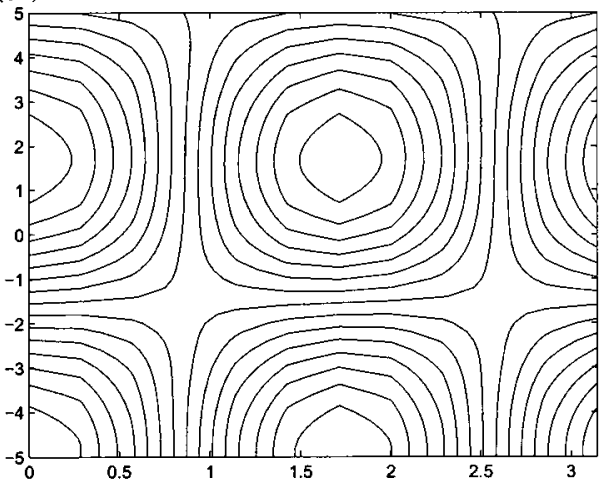

Figure 5.10: Linear simulation of Rossby wave propagation $(\varepsilon=0)$ with the time-dependent radiation condition in the long-wave limit $\delta=0$ : contour plots of the streamfunction $\psi(x, z, t)$ obtained at (a) $t=2$, (b) $t=5$, (c) $t=10$, (d) $t=23$, (e) $t=45$ and (f) $t=62$. A periodic forcing $e^{i k_{o} x}, k_{o}=2$, has been applied at the upper boundary of the rectangular domain and $\beta=1$. The background flow is constant, $\bar{u}=1$ 
(a) $t=10$

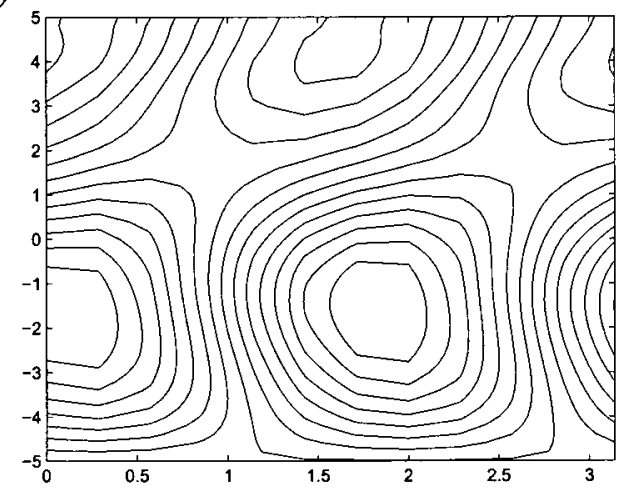

(c) $t=42$

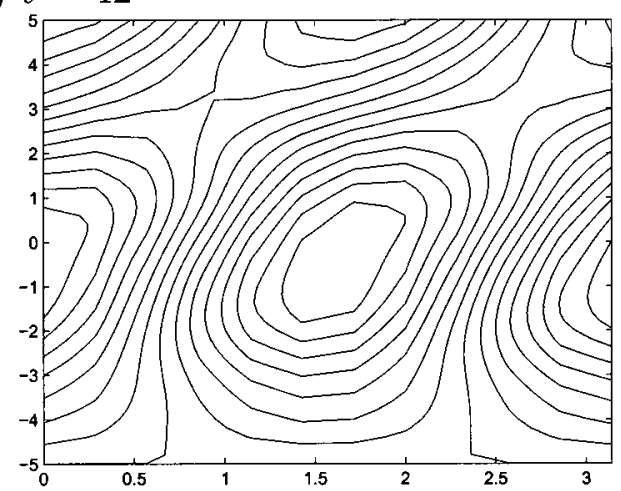

(e) $t=80$

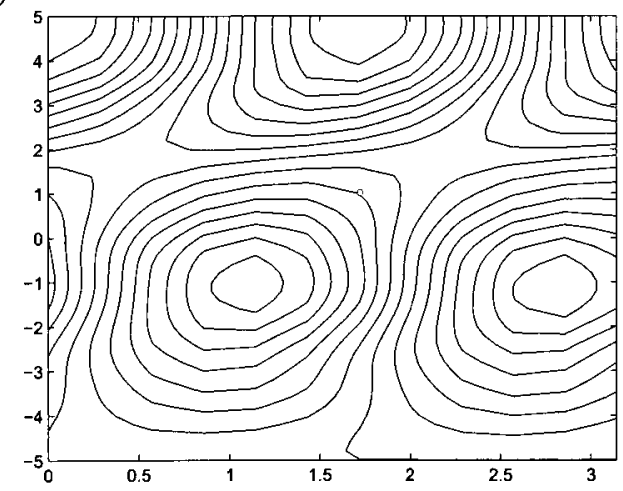

(b) $t=23$

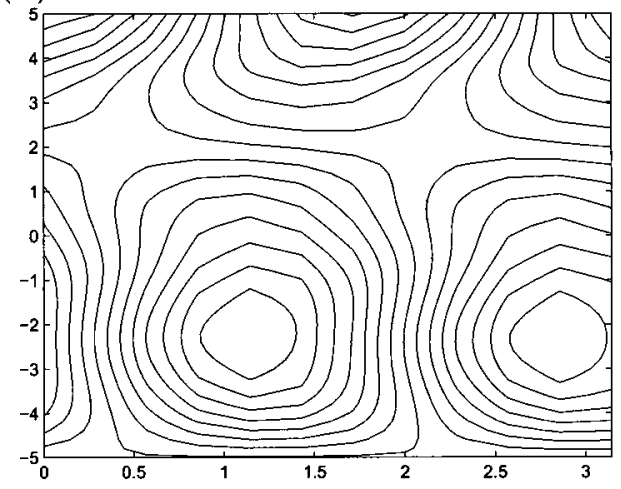

(d) $t=62$

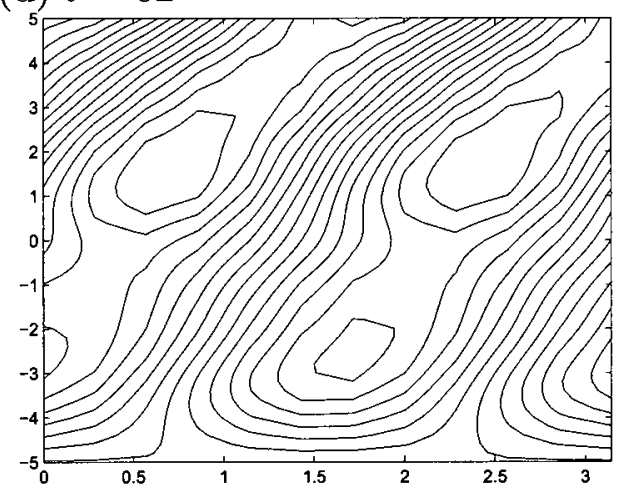

(f) $t=85$

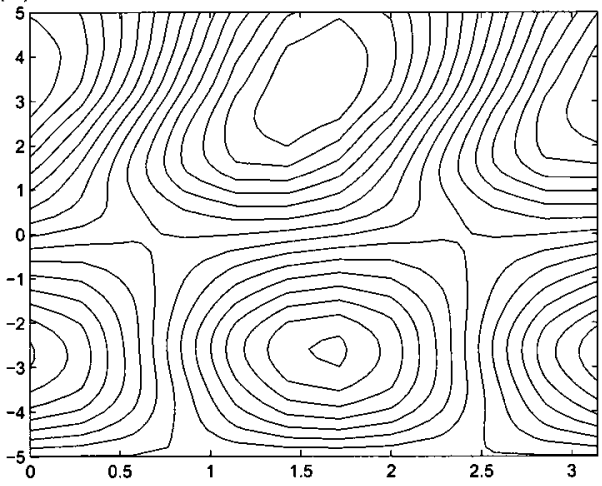

Figure 5.11: Nonlinear simulation of Rossby wave propagation $(\varepsilon=0.05)$ with the zero boundary condition: contour plots of the streamfunction $\psi(x, z, t)$ obtained at (a) $t=10$, (b) $t=23$, (c) $t=42$, (d) $t=62$, (e) $t=80$ and (f) $t=85$. A periodic forcing $e^{i k_{o} x}, k_{o}=2$, has been applied at the upper boundary of the rectangular domain, $\delta=0.2$ and $\beta=1$. The background flow is constant, $\bar{u}=1$. 
(a) $t=10$

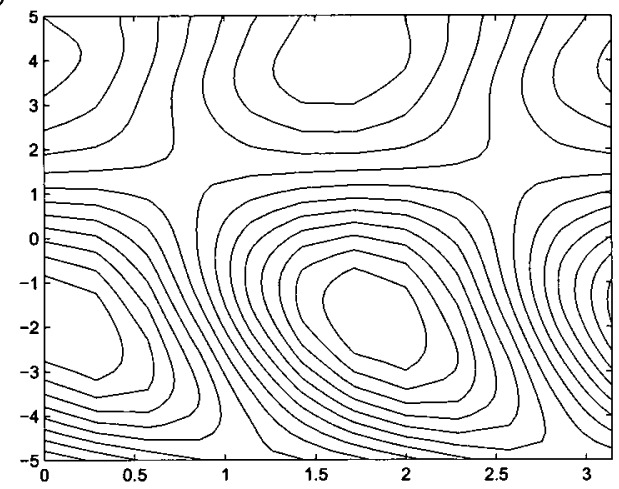

(c) $t=42$

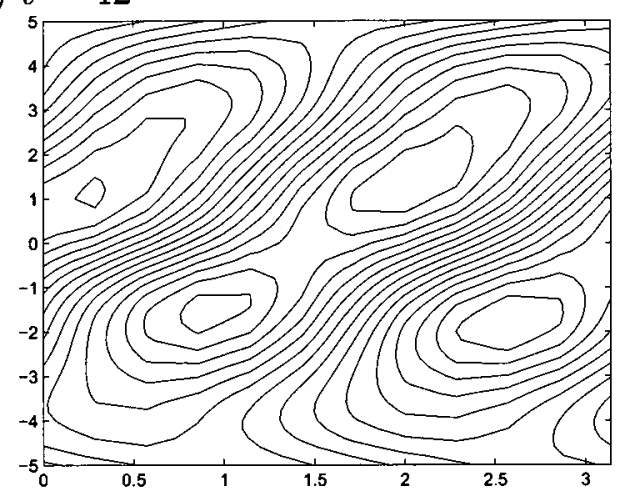

(e) $t=67$

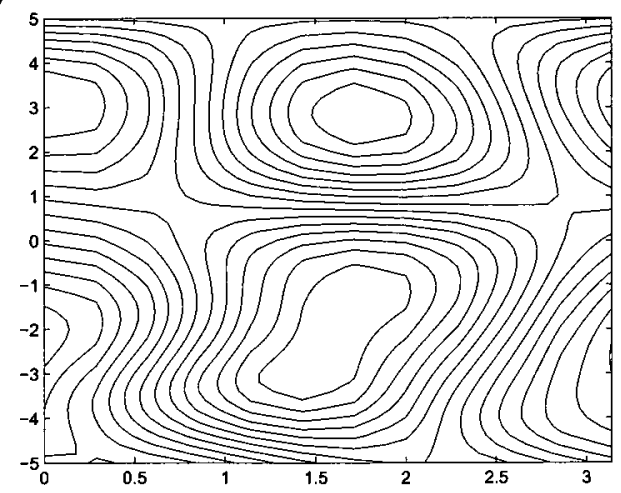

(b) $t=23$

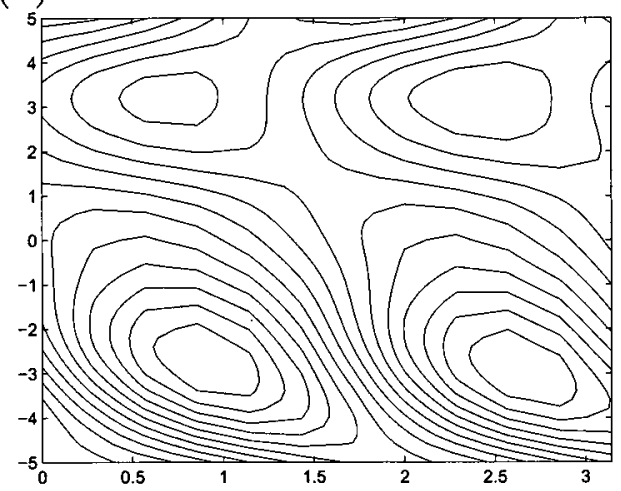

(d) $t=62$

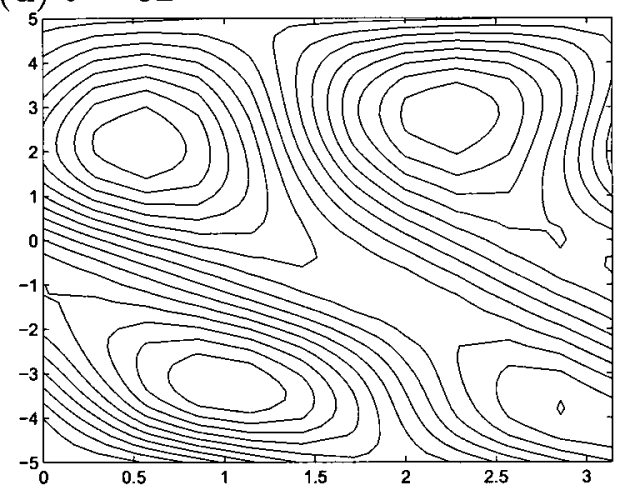

(f) $t=69$

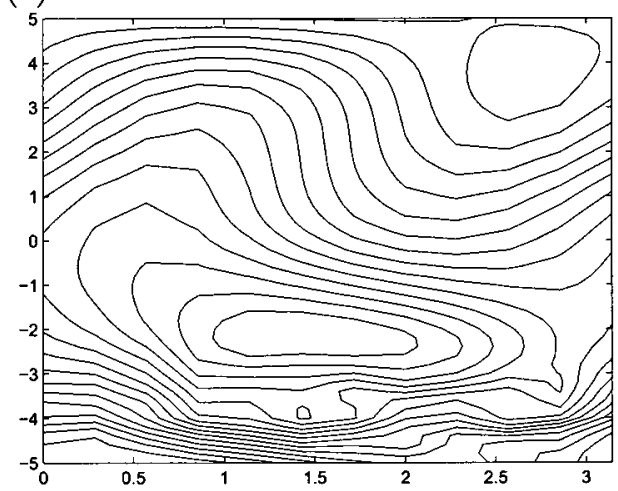

Figure 5.12: Nonlinear simulation of Rossby wave propagation $(\varepsilon=0.05)$ with the steady radiation condition, contour plots of the streamfunction $\psi(x, z, t)$ obtained at (a) $t=10$, (b) $t=23$, (c) $t=42$, (d) $t=62$, (e) $t=67$ and (f) $t=69$. A periodic forcing $e^{i k_{o} x}, k_{o}=2$, has been applied at the upper boundary of the rectangular domain, $\delta=0.2$ and $\beta=1$. The background flow is constant, $\bar{u}=1$. 
(a) $t=10$

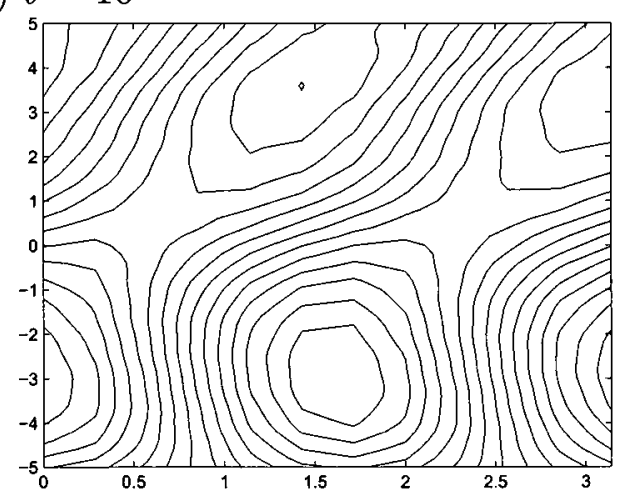

(c) $t=42$

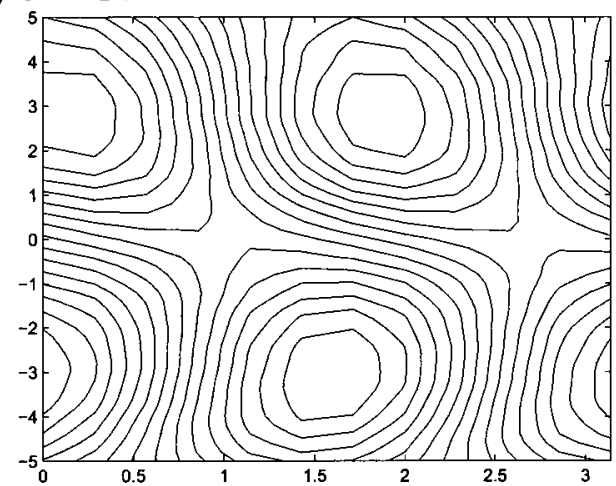

(e) $t=80$

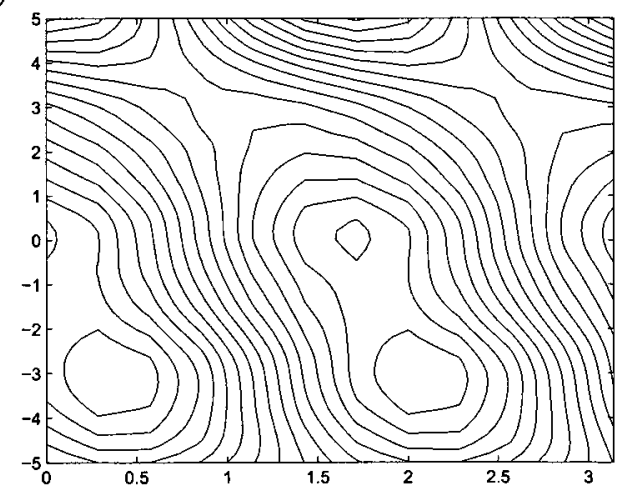

(b) $t=23$

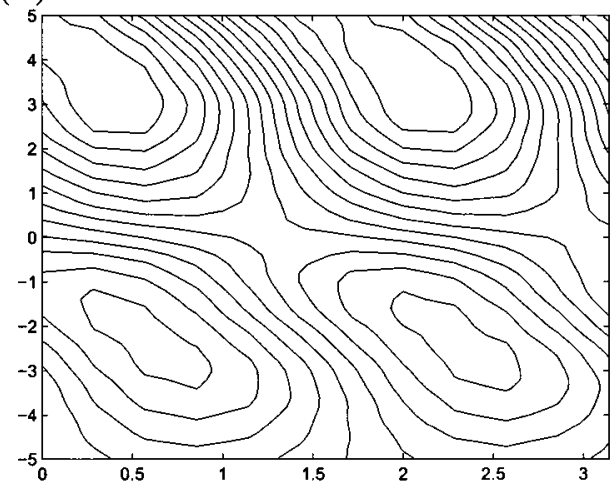

(d) $t=62$

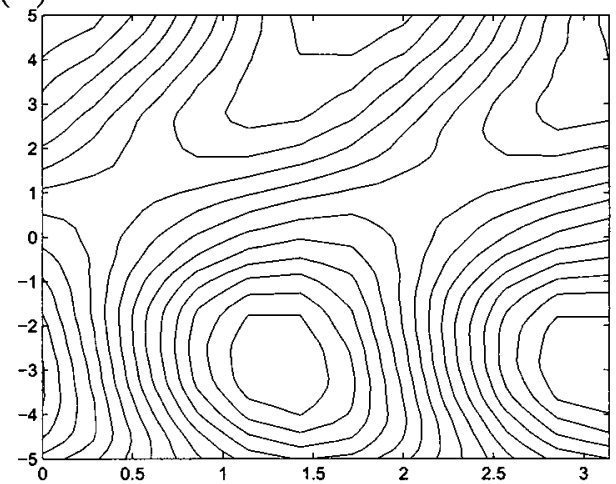

(f) $t=85$

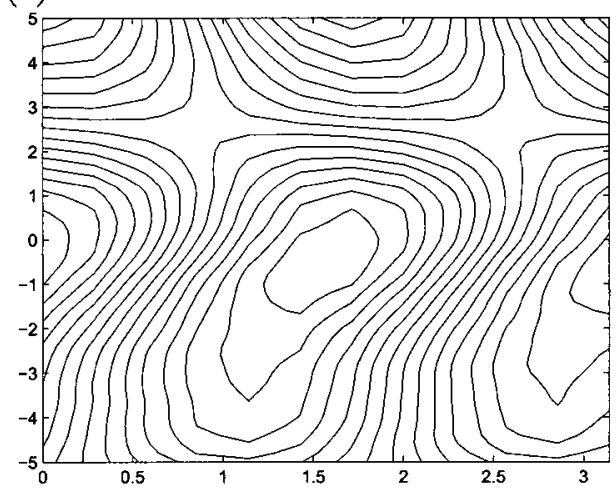

Figure 5.13: Nonlinear simulation of Rossby wave propagation $(\varepsilon=0.05)$ with the time-dependent radiation condition: contour plots of the streamfunction $\psi(x, z, t)$ obtained at (a) $t=10$, (b) $t=23$, (c) $t=42$, (d) $t=62$, (e) $t=80$ and (f) $t=85$. A periodic forcing $e^{i k_{o} x}, k_{o}=2$, has been applied at the upper boundary of the rectangular domain, $\delta=0.2$ and $\beta=1$. The background flow is constant, $\bar{u}=1$. 


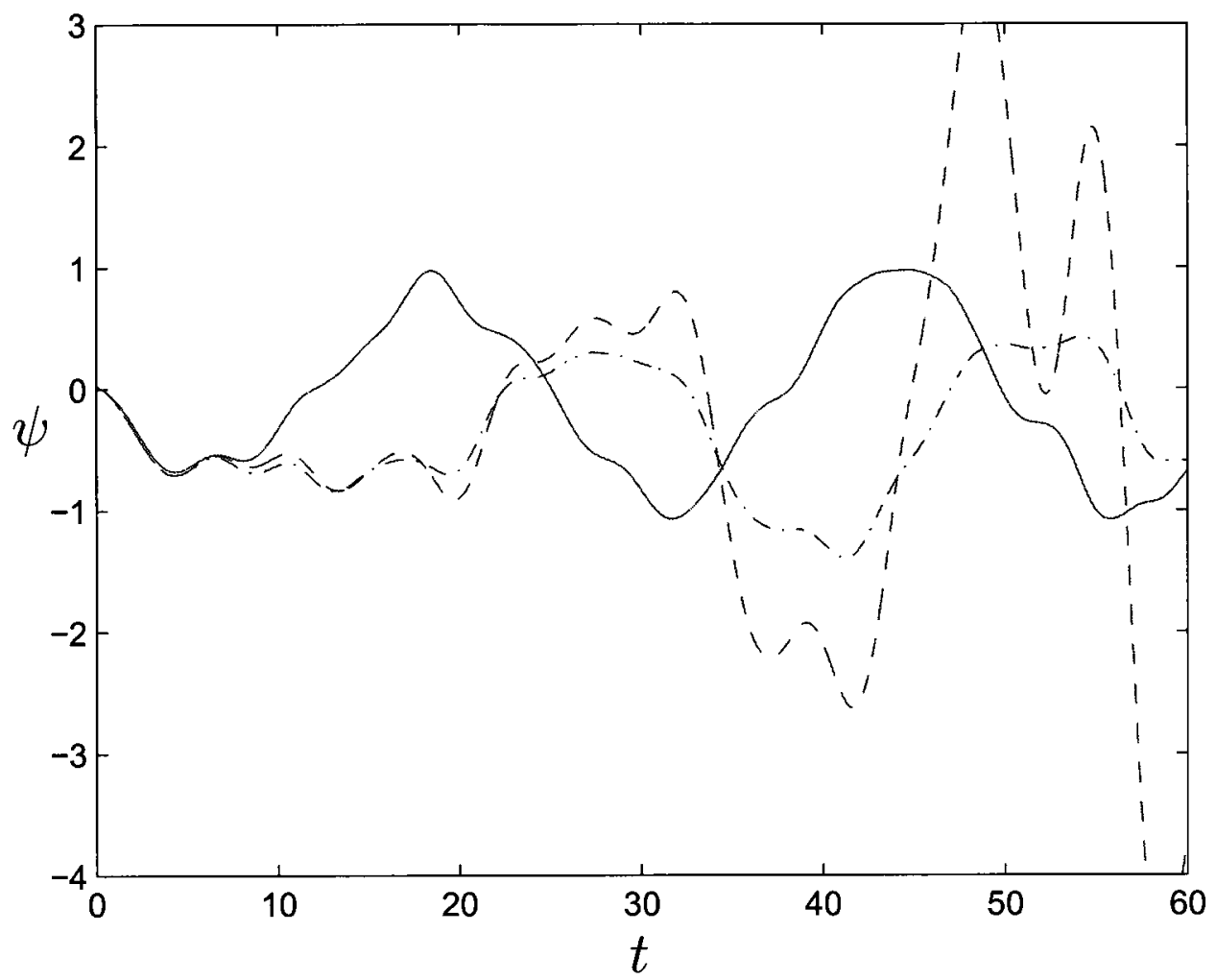

Figure 5.14: Nonlinear simulation of Rossby wave propagation $(\varepsilon=0.05)$ : plot of the streamfunction $\psi(x, y, t)$ as a function of time t at $x=0.6$ and $y=-1$, $0 \leq t \leq 60$. A periodic forcing $e^{i k_{o} x}, k_{o}=2$, has been applied at the upper boundary of the rectangular domain, $\delta=0.2$ and $\beta=1$. The background flow is constant, $\bar{u}=1$. The solid line corresponds to the simulation with time-dependent radiation condition and the dashed-dotted line corresponds to the simulation with the zero boundary condition whereas the dashed line is the result obtained for the simulation with the steady radiation condition. 
(a)

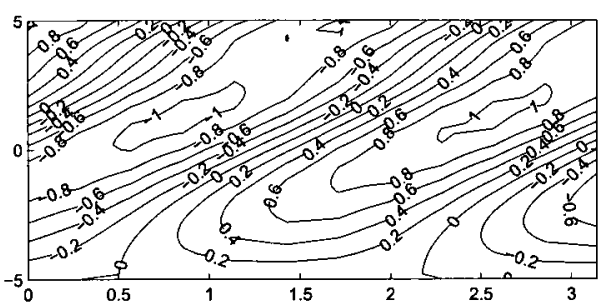

(b)

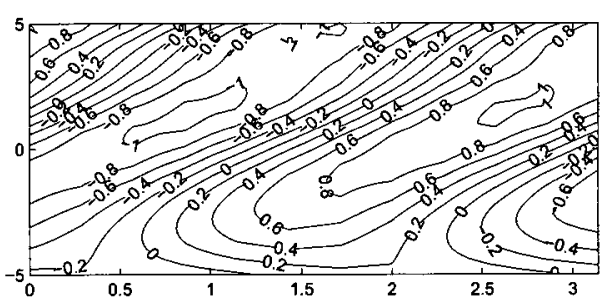

(c)

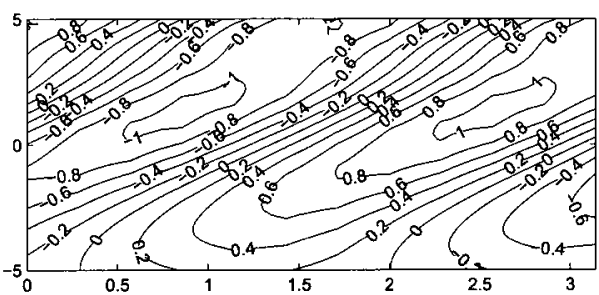

(d)

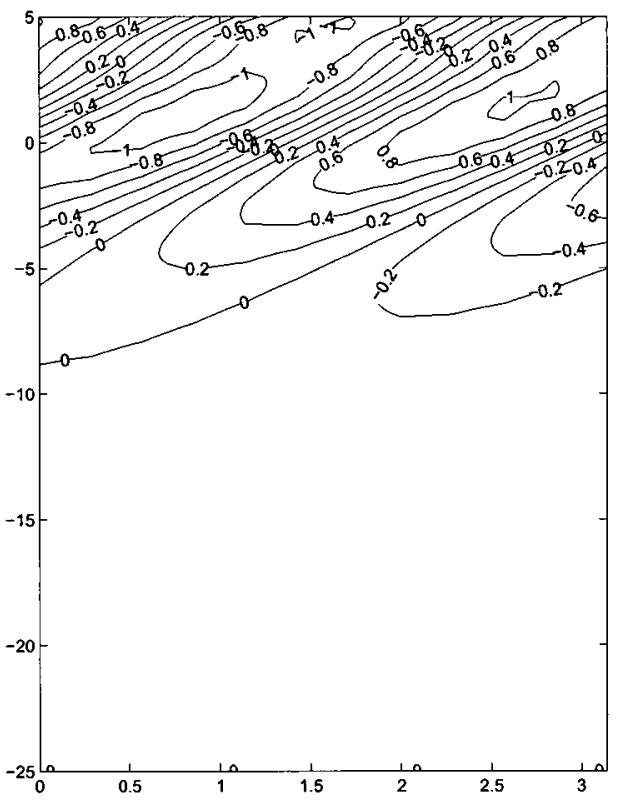

Figure 5.15: Nonlinear simulation of Rossby wave propagation $(\varepsilon=0.05)$ : contours plot of the streamfunction $\psi(x, y, t)$ at the time $t=5 \mathrm{with}$, (a) the zero boundary condition, (b) the steady radiation condition, (c) the time-dependent radiation condition and (d) the zeros boundary condition on a large domain in the $y$-direction. A periodic forcing $e^{i k_{o} x}, k_{o}=2$, has been applied at the upper boundary of the rectangular domain and $\delta=0.2$. The background flow is constant, $\bar{u}=1$. 


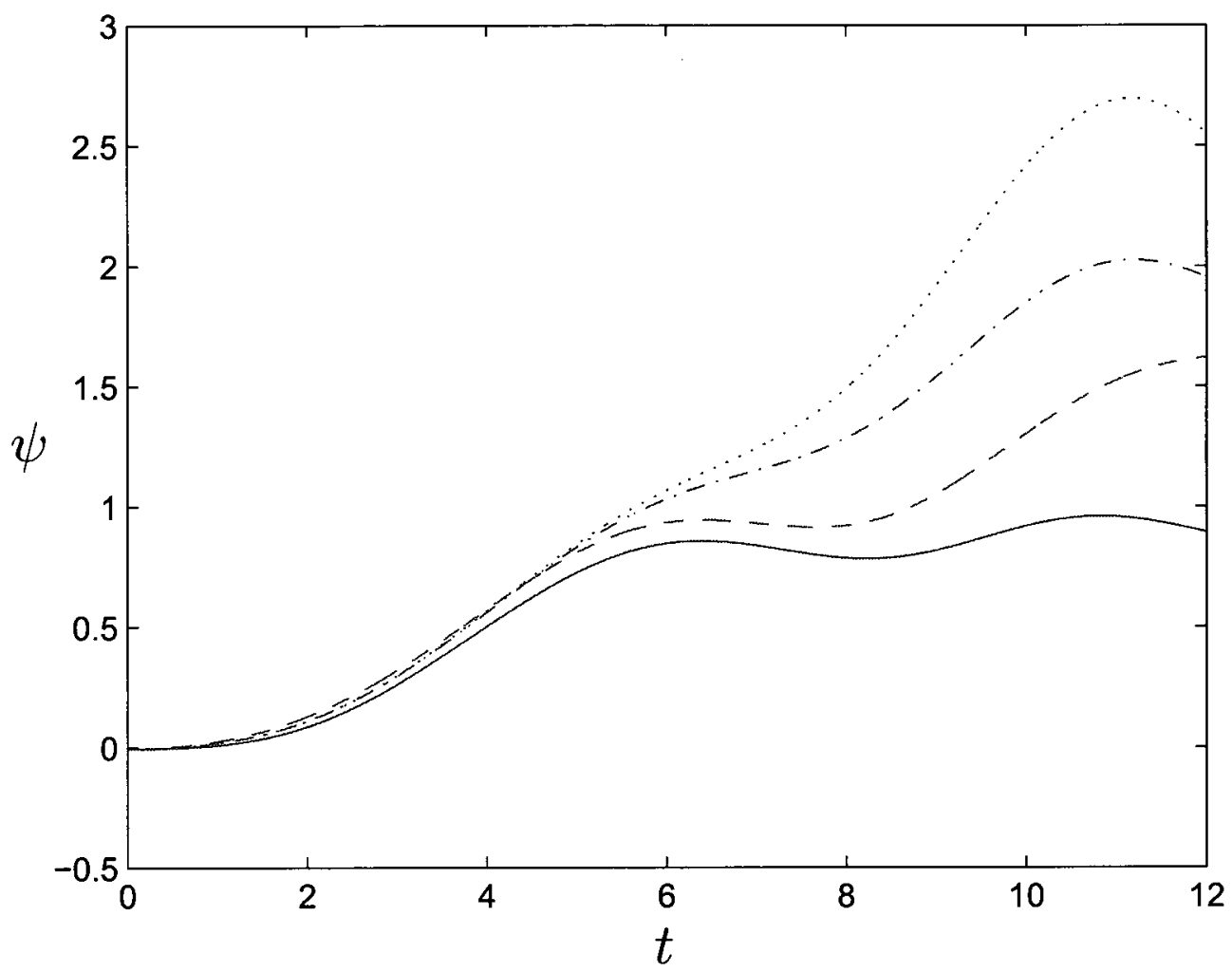

Figure 5.16: Nonlinear simulation of Rossby wave propagation $(\varepsilon=0.05)$ : plot of the streamfunction $\psi(x=1.5, y=-1, t)$ as a function of time $t, 0<t<12$. A periodic forcing $e^{i k_{o} x}, k_{o}=2$, has been applied at the upper boundary of the rectangular domain and $\delta=0.2$. The background flow is constant, $\bar{u}=1$. The solid line corresponds to the zero boundary condition on a large domain in the $y$-direction, the dashed line corresponds to the time-dependent radiation condition, the dotted line corresponds to the steady radiation condition and the dashed-dotted line to the zero boundary condition. 


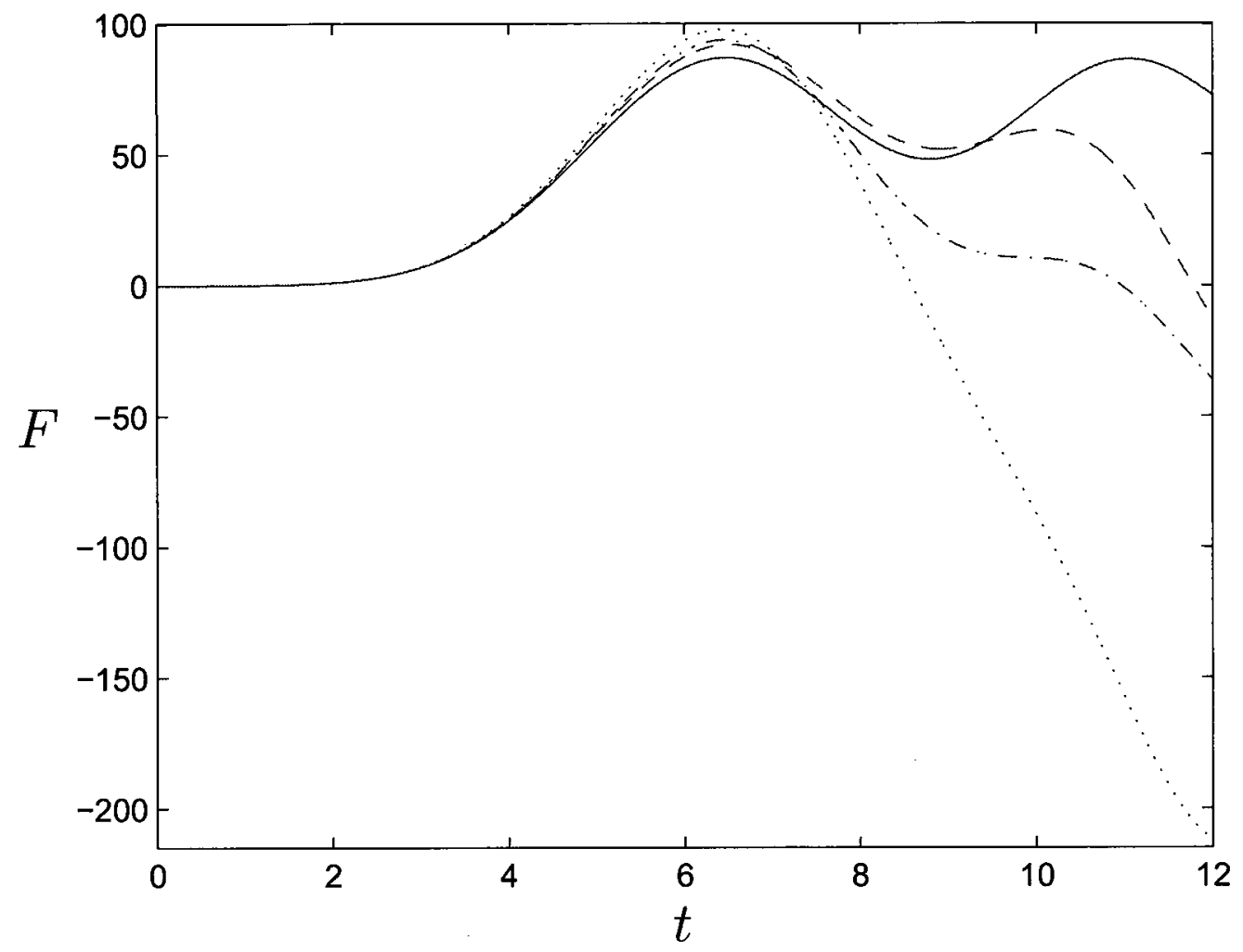

Figure 5.17: Nonlinear simulation of Rossby wave propagation $(\varepsilon=0.05)$ : plot of the averaged momentum flux $F(y=-1, t)$ as a function of time $t, 0<t<12$. A periodic forcing $e^{i k_{o} x}, k_{o}=2$, has been applied at the upper boundary of the rectangular domain and $\delta=0.2$. The background flow is constant, $\bar{u}=1$. The solid line corresponds to the zero boundary condition on a large domain in the $y$-direction, the dashed line corresponds to the time-dependent radiation condition, the dotted line corresponds to the steady radiation condition and the dashed-dotted line to the zero boundary condition.

Finally we present results obtained by adding a nonlinear correction to the time-dependent radiation condition as described in section 3.3. Recall that the nonlinear correction was made only to the zero wavenumber component of the solution. The expressions for the nonlinear corrections needed for the other wavenumber components given by equation (3.66) are to complicated to be eval- 
uated exactly. The effect of adding a nonlinear correction for $\kappa=0$ to the time-dependent linear radiation condition can be examined by looking at the curves of $\hat{\psi}$ as a function of the wanumber $\kappa$ in Figure 5.18. The contour plots of the streamfunction with the nonlinear (not shown) correction look very similar to those shown in Figure 5.13 it seems there is no significant difference. In contrast we see that in the corresponding gravity wave problem the zero wavenumber term and the nonlinear correction are significantly large.

Figure 5.18 illustrates the fact that in the Rossby wave problem the magnitude of the zero wavenumber component $\hat{\psi}(0, y, t)$ is very small compared with the magnitude of the forced wave $\hat{\psi}\left( \pm k_{o}, y, t\right)$. The graphs shown are for $y=-4.8$ near the outflow boundary which is at $y=-5$ and $t=125$. The solid line shows $|\hat{\psi}|$ as a function of $\kappa$ computed without the nonlinear correction. For $\kappa= \pm k_{o}|\hat{\psi}|=8.23$ and for $\kappa=0|\hat{\psi}|=0.14$. The dashed curve shows $|\hat{\psi}|$ computed with the nonlinear correction. In this case $|\hat{\psi}|=8.23$ for $\kappa= \pm k_{o}$ and $|\hat{\psi}|=0.10$ for $\kappa=0$. Since the difference is very small then the difference between the 2 curves is barely noticeable. 


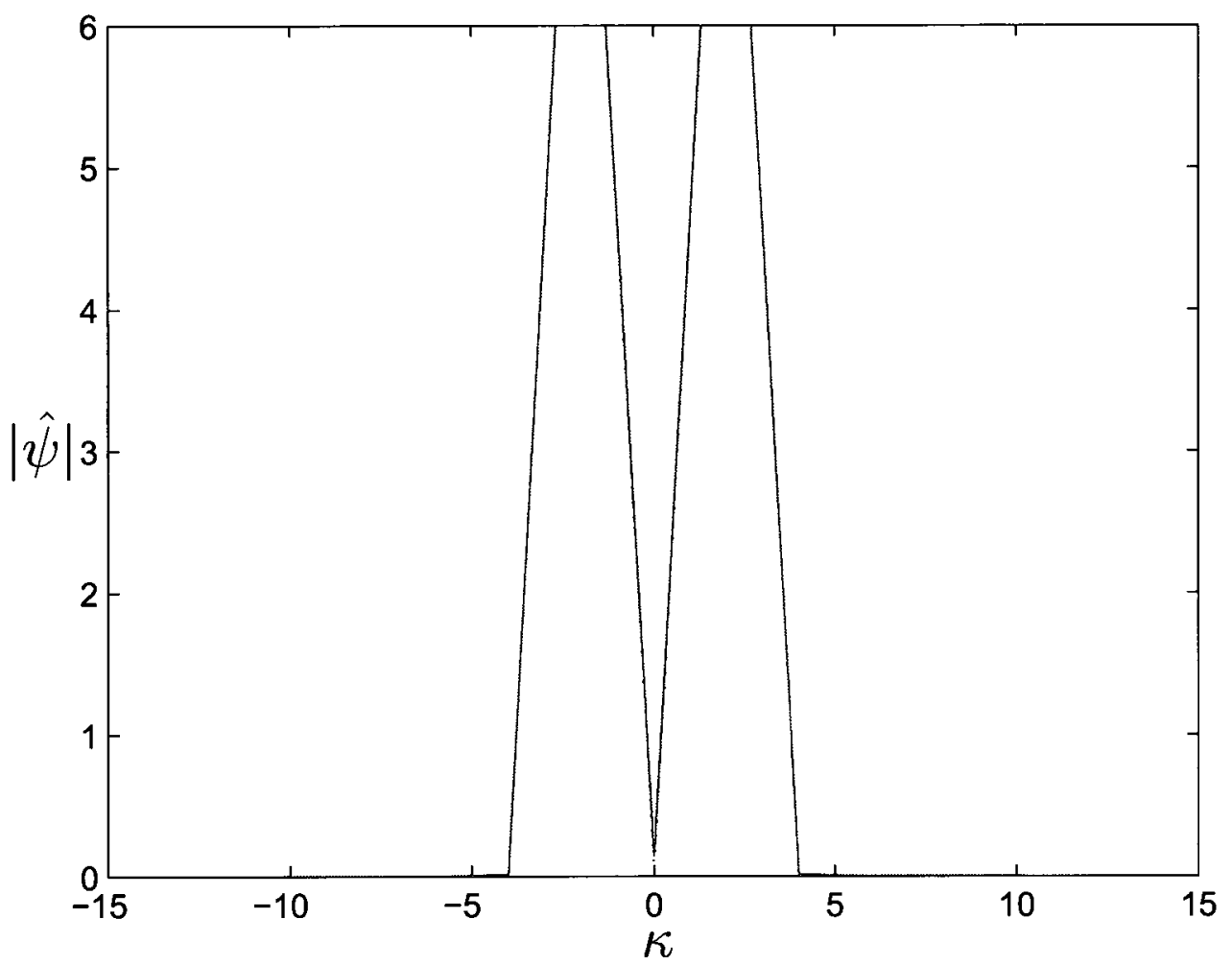

Figure 5.18: Nonlinear simulation of Rossby wave propagation $(\varepsilon=0.05)$ with the corrected time-dependent radiation condition: plot of $\hat{\psi}(\kappa, y, t)$ as a function of the wavenumber $\kappa$ at the time $t=125$ across the outflow boundary . A periodic forcing $e^{i k_{o} x}, k_{o}=2$, has been applied at the upper boundary of the rectangular domain, $\delta=0.2$ and $\beta=1$. The background flow is constant, $\bar{u}=1$. The solid line corresponds to the simulation with the time-dependent radiation condition without the nonlinear correction while the dashed line corresponds to the simulation with the time dependent radiation condition with the nonlinear correction. The difference between the two curve is barely noticeable at $(\kappa=0)$ since the zero wavenumber term and the nonlinear correction are very small compared with the magnitude of of $\hat{\psi}$ at the forcing $\left(\kappa= \pm k_{o}\right)$. 


\subsection{The results of simulations of gravity wave propagation}

For the gravity wave problem given by equations (4.23) to (4.28) simulations are carried out on a rectangular domain given by $0 \leq x \leq 2 \pi$ and $0 \leq z \leq 10$. The wave source is at $z_{1}=0$ and has the form

$$
\psi\left(x, z_{1}, t\right)=2 \cos k_{o} x=e^{i k_{o} x}+c . c .
$$

We used the following nondimensional parameters; $\varepsilon=0.05$, the Prandtl number $\operatorname{Pr}=0.72$ and $\delta=0.2$. The scale height was set to $H_{o}=4.9$ so that the Brunt Väisälä frequency $N=\sqrt{2}$. According to the Miles-Howard theorem (Appendix C), the Richardson number is the measure of the stability of the stratified flow. In this study the Richardson number $R i$ was chosen to be greater than a $1 / 4$ to make sure the flow is stable according to Miles-Howard's theorem. In the first set of simulations the background flow is set to $\vec{u}=\tanh z-z_{c}$ so that the critical layer is located at $z_{c}=5$ as shown in Figure 5.19 . The Richardson number depends on $z$ and its minimum value is $R i_{c}=2$ at $z_{c}=5$. In the rest of the simulations we set $\bar{u}=1$. So the Richardson is infinite. A small amount of viscosity corresponding to a kinematic viscosity of $\nu=3 \cdot 10^{-4}$ was necessary to prevent the accumulation of wave activity at the high end of the wavenumber spectrum and near the forced boundary in the nonlinear simulations. 


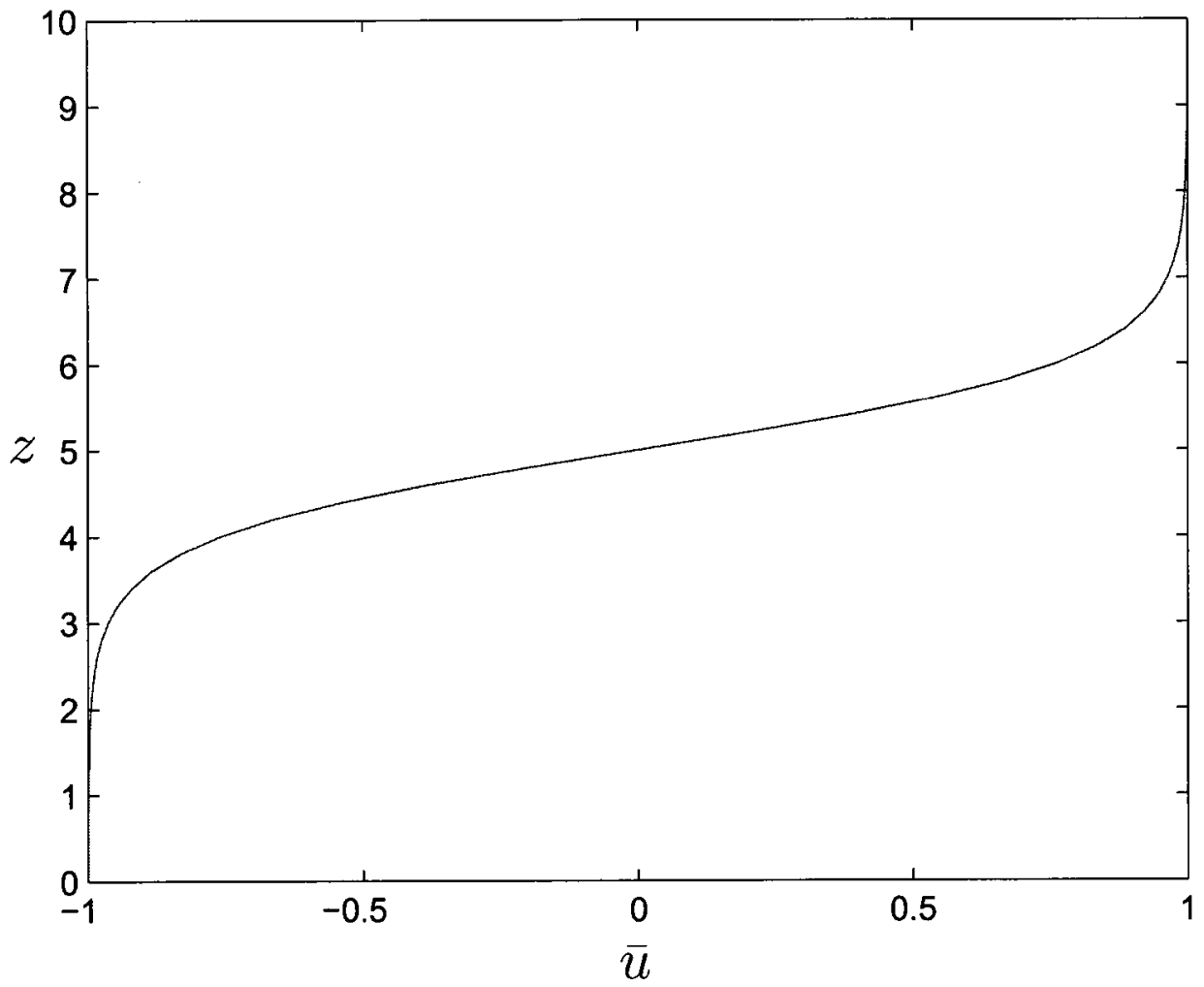

Figure 5.19: Simulation of gravity wave propagation: the background flow speed is $\bar{u}=\tanh \left(z-z_{c}\right)$. The critical level is at $z_{c}=5$. 


\subsubsection{The results of simulations of gravity wave propaga- tions in a configuration with a critical layer in the flow}

We solve the initial boundary value problem (4.23) to (4.28) using the numerical methods described in Chapter 4. Simulations for both linear and nonlinear problems were carried out over the nondimensional time interval from $t=0$ to $t=34$ and the results are presented in Figures 5.20-5.21. Figure 5.20(a) is the contour plot of the streamfunction which results from the linear simulation and $5.20(\mathrm{~b})$ is the result of the nonlinear simulation. In both Figure 5.20(a) and Figure $5.20(\mathrm{~b})$ there is not much propagation wave activity above the critical layer. Figure 5.21 is the plot of the jump in the horizontal momentum flux as a function of time, the dashed line correspond to the linear simulation whereas the solid line corresponds to the nonlinear simulation. In the linear problem $[F]$ becomes almost constant by about $t=20$ indicating absorption while in the nonlinear problem $[F]$ starts to decrease arounf $t=20$ and attains negative values indicating reflection then again becomes positive indicating absorption. Similar results were obtained by Campbell and Maslowe (2003). This confirms that our programs work properly. 

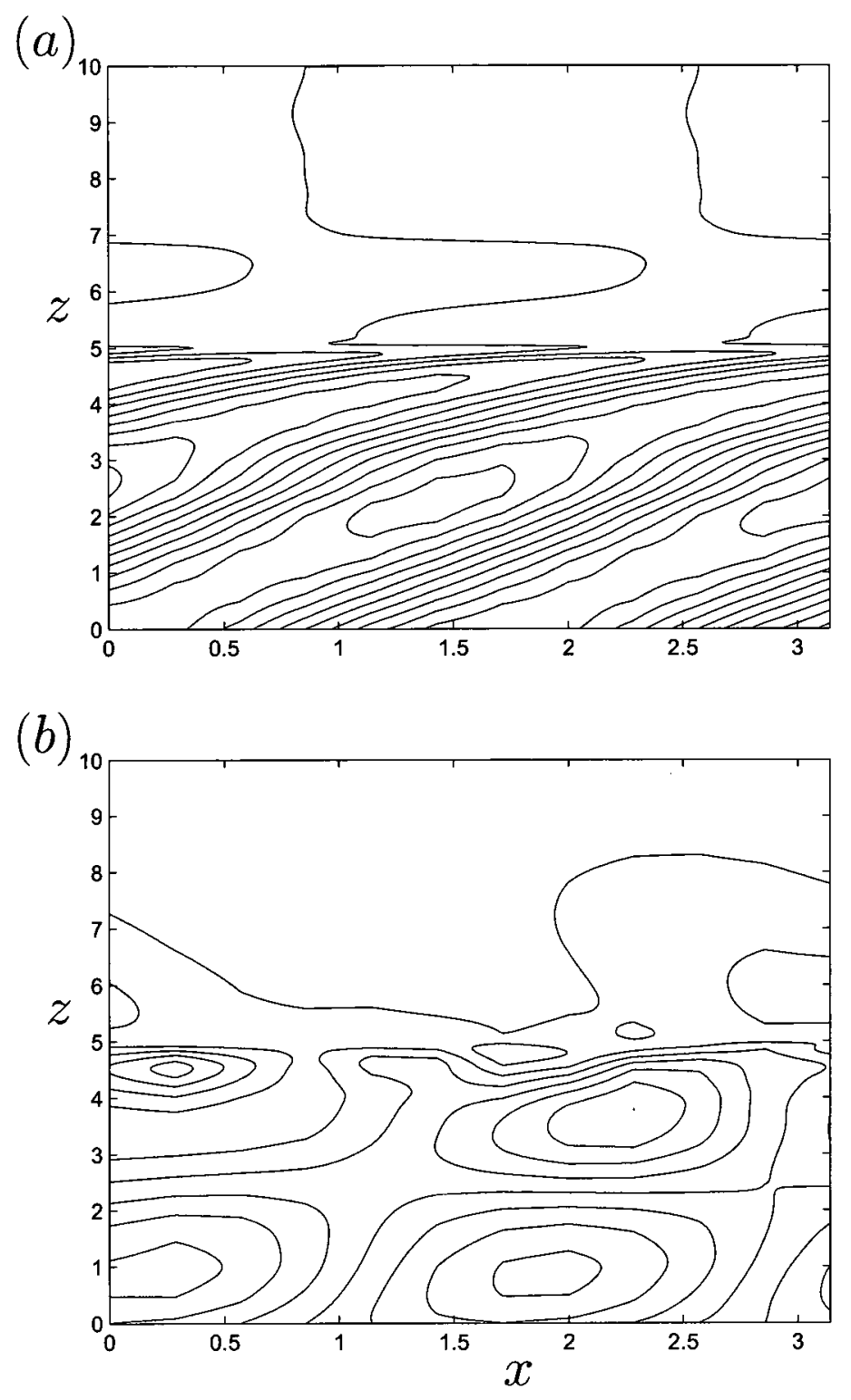

Figure 5.20: Linear and nonlinear simulations of gravity wave propagation: contour plot of $\Psi(x, z, t)$ at $t=34,(a)$ linear simulation with $\epsilon=0$ and $(b)$ nonlinear simulation with $\epsilon=0.05$. In both $(a)$ and $(b) \delta=0.2$ and $R i=2$. A periodic forcing $e^{i k_{o} x}, k_{o}=2$, has been applied at the lower boundary of the rectangular domain. The background flow speed is $\bar{u}=\tanh \left(z-z_{c}\right)$, the critical level is at $z_{c}=5$. Both contour plots show that there is not much transmission of wave activity beyond the critical layer. 


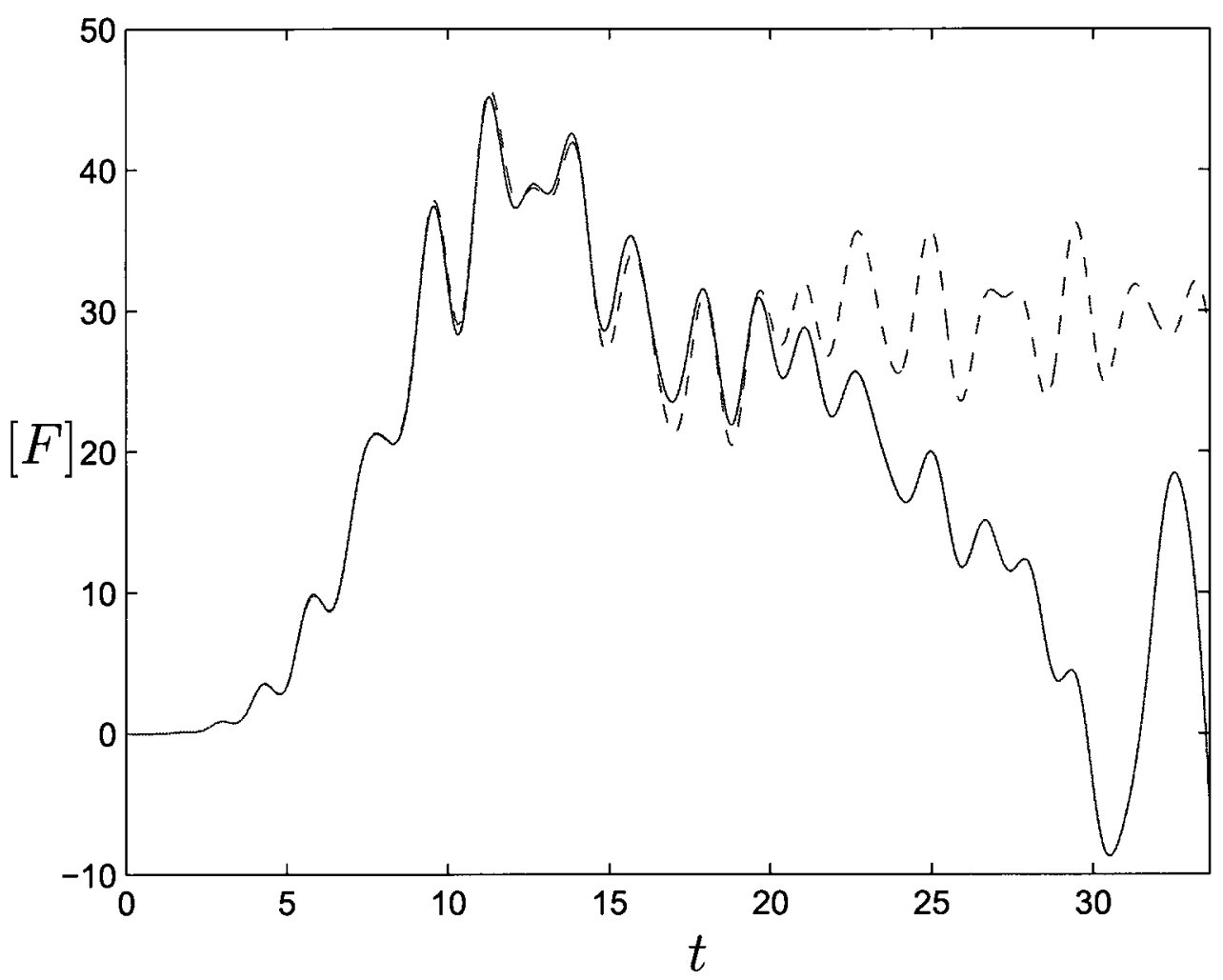

Figure 5.21: Linear and nonlinear simulations of gravity wave propagation: plot of the jump in the momentum flux $[F]$ across the critical layer as a function of time for $0 \leq t \leq 34$. A periodic forcing $e^{i k_{o} x}, k_{o}=2$, has been applied at the lower boundary of the rectangular domain, $\delta=0.2$ and $R i=2$. The background flow speed is $\bar{u}=\tanh \left(z-z_{c}\right)$, the critical level is at $z_{c}=5$. The dashed line shows the result obtained for the linear simulation $(\varepsilon=0)$ while the solid line shows the result obtained for the nonlinear simulation $(\varepsilon=0.05)$. In the linear case $[F]$ becomes almost constant by about $t=20$. This indicates absorption of the waves by the mean flow. In the nonlinear case $[F]$ alternates between the positive values (absorption) and the negative values (reflection). 


\subsubsection{Results of simulations of gravity wave propagations in a configuration without a critical layer in flow}

Results of both linear and nonlinear simulations of gravity waves propagation are shown in Figures 5.22-5.29. Figures 5.22, 5.23, 5.26 and 5.24 are the contour plots obtained as results of the linear simulations of the gravity wave at different time $t=\{4,8,15,35,45,65\}$. These contour plots are almost similar up to $t=15$, but significant differences between these contour plots are observed around $t=30$.

Figure 5.22 shows the results obtained with the zero boundary condition. If the zero boundary condition is implemented at the outflow boundary, there is a huge difference between contour plots of the streamfunction $\psi(x, z, t)$ at $t=15$ and $t=35$, these differences keep developing even at large time $t=45$ and $t=65$ as a result of reflection and interference of incident and reflected waves as shown in Figure 5.22.

Figure 5.23 shows the results obtained with the steady radiation condition. However if the steady radiation condition is implemented again there are deformations of the contour plots as in the Rossby wave problem. The direction of propagation of the waves is fixed near the outflow boundary because the vertical wavelength is fixed in the radiation condition.

The contour plots in Figure 5.24 are the results of the linear simulations if the time-dependent radiation condition is applied at the outflow boundary. In this case the waves evolve with time near outflow boundary. This is explained by the fact that by implementing the time-dependent radiation condition we take into account the transient evolution of the gravity wave inside the rectangular domain. Figure 5.25 shows that if the time-dependent radiation condition is applied at the outflow boundary the horizontal momentum flux is fairly constant 
and almost independent of the altitude $z$ as expected from the Eliassen-Palm theorem.

The contour plots shown in Figure 5.26 are the results of the linear simulations in the long-wave limit $\delta=0$. For $t \leq 15$ the contour plots of the time-dependent radiation condition resembles to the contour plots of the timedependent radiation condition in the long-wave limit $\delta=0$. As seen in Figure $5.26(\mathrm{~d})$ at later time $(t \gtrsim 35)$ the solution reaches a steady state and is almost exactly periodic in the $z$-direction as predicted by Nadon and Campbell (2007). 
(a) $t=4$

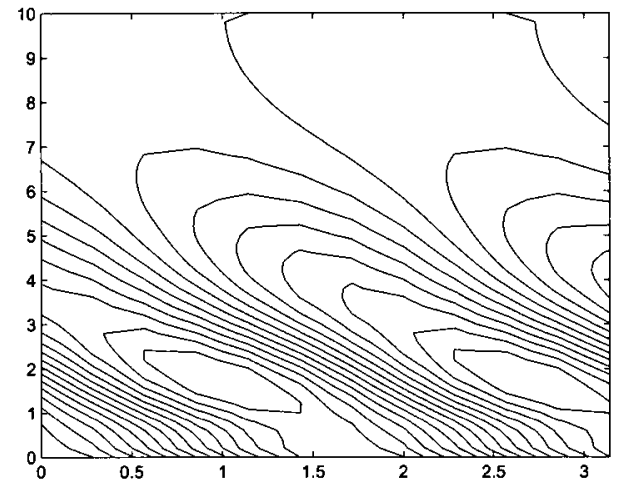

(c) $t=15$

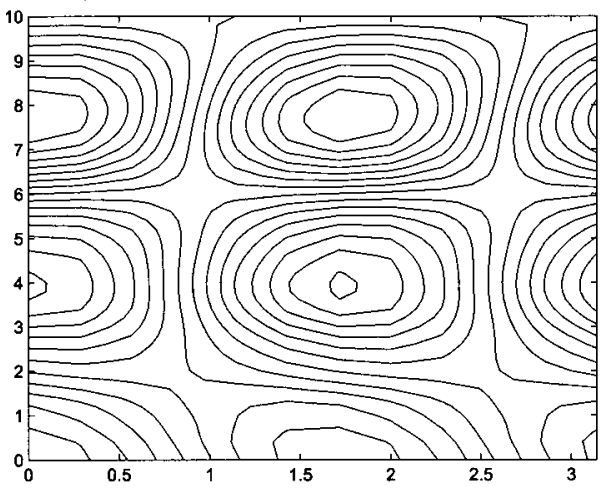

(e) $\mathrm{t}=45$

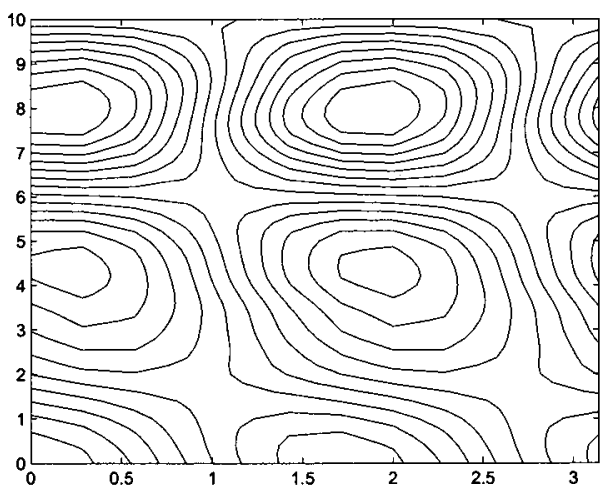

(b) $\mathrm{t}=8$

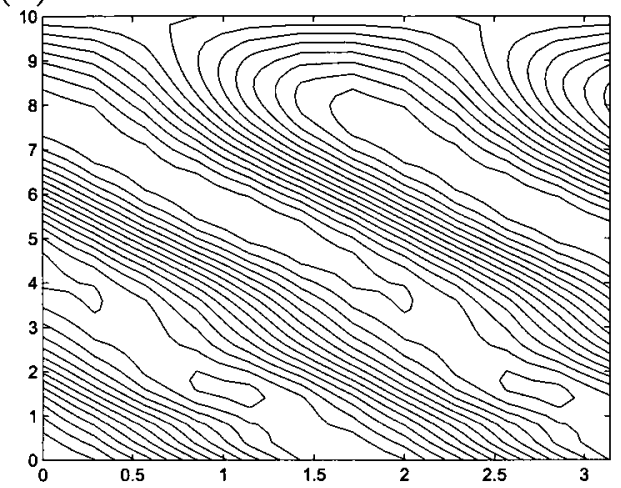

(d) $\mathrm{t}=35$

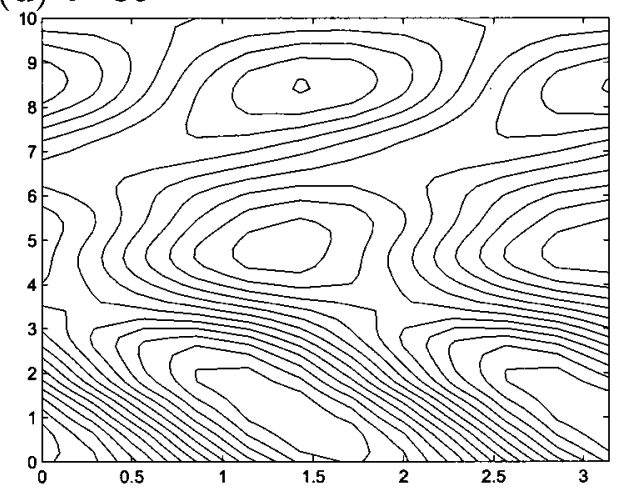

(f) $\mathrm{t}=65$

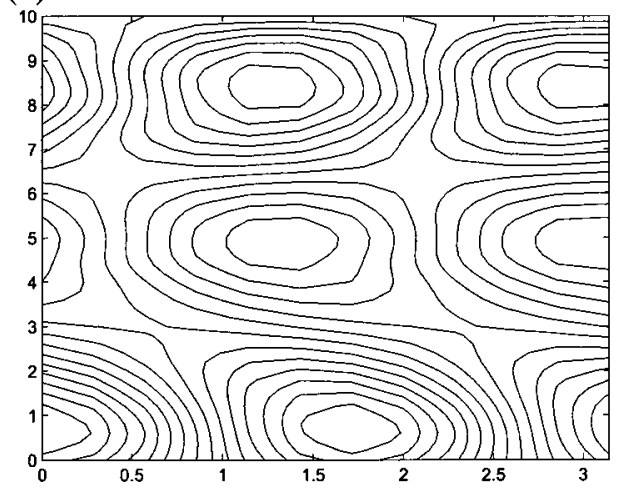

Figure 5.22: Linear simulation of gravity wave propagation $(\varepsilon=0)$ with the zero boundary condition: contour plots of the streamfunction $\psi(x, z, t)$ obtained at (a) $t=4$, (b) $t=8$, (c) $t=15$, (d) $t=35$, (e) $t=45$ and (f) $t=65$. A periodic forcing $e^{i k_{o} x}, k_{o}=2$, has been applied at the lower boundary of the rectangular domain and $\delta=0.2$. The background flow is constant, $\bar{u}=1$. 
(a) $t=4$

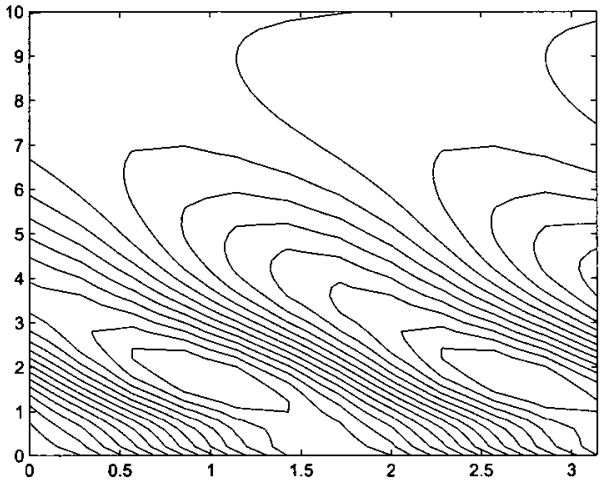

(c) $t=15$

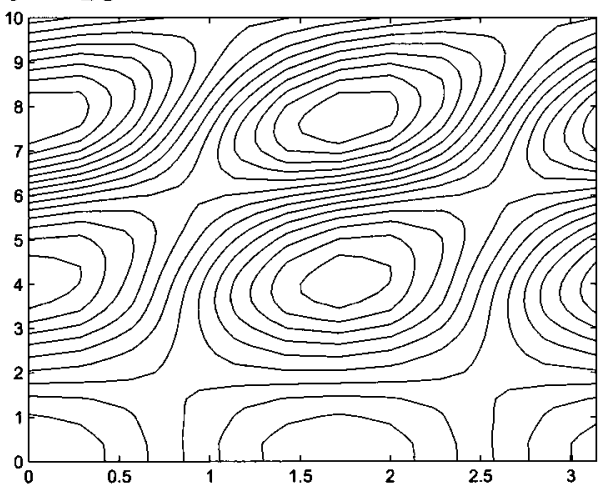

(e) $t=45$

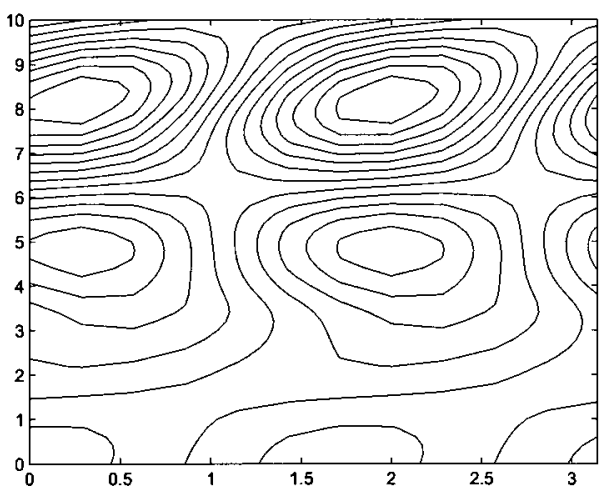

(b) $t=8$

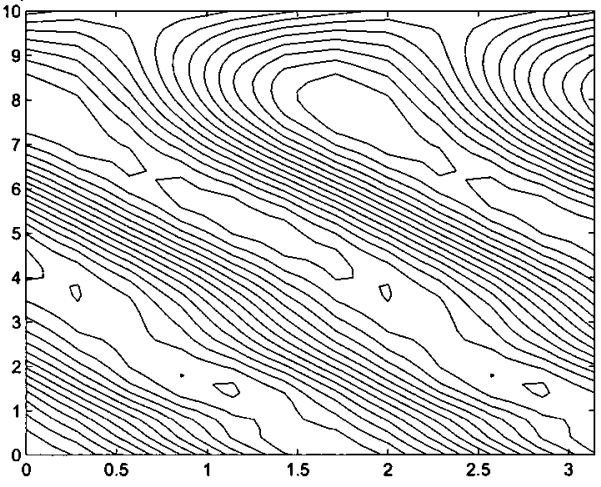

(d) $t=35$

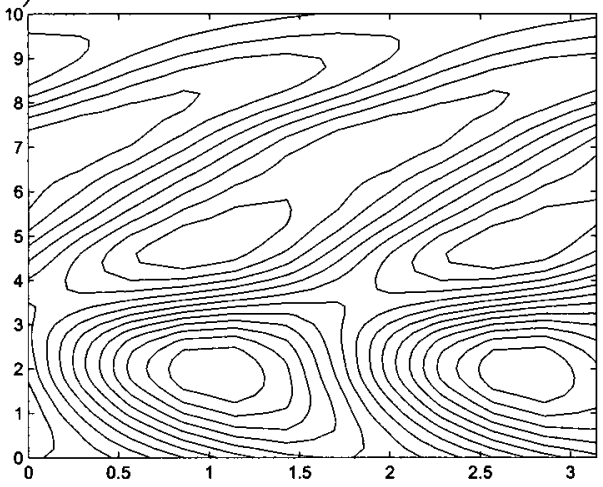

(f) $t=65$

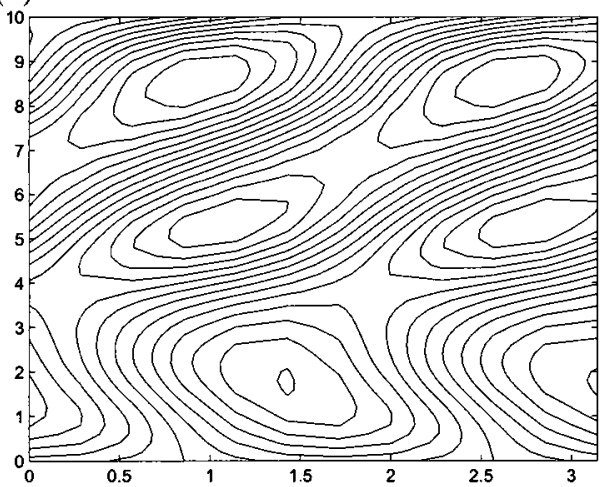

Figure 5.23: Linear simulation of gravity wave propagation $(\varepsilon=0)$ with the steady boundary condition: contour plots of the streamfunction $\psi(x, z, t)$ obtained at (a) $t=4$, (b) $t=8$, (c) $t=15$, (d) $t=35$, (e) $t=45$ and (f) $t=65$. A periodic forcing $e^{i k_{o} x}, k_{o}=2$, has been applied at the lower boundary of the rectangular domain and $\delta=0.2$. The background flow is constant, $\bar{u}=1$. 
(a) $t=4$

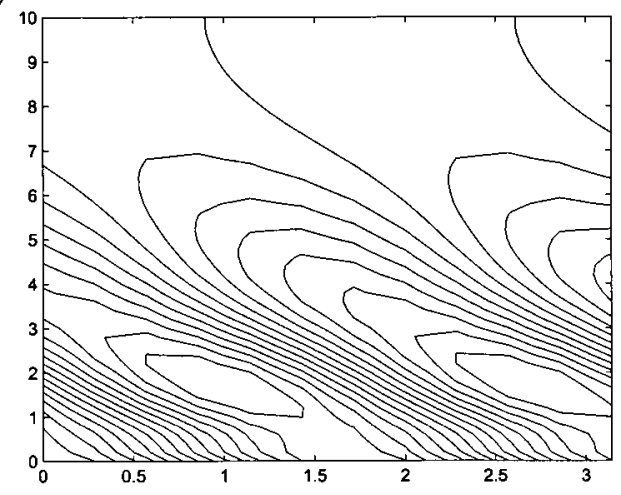

(c) $t=15$

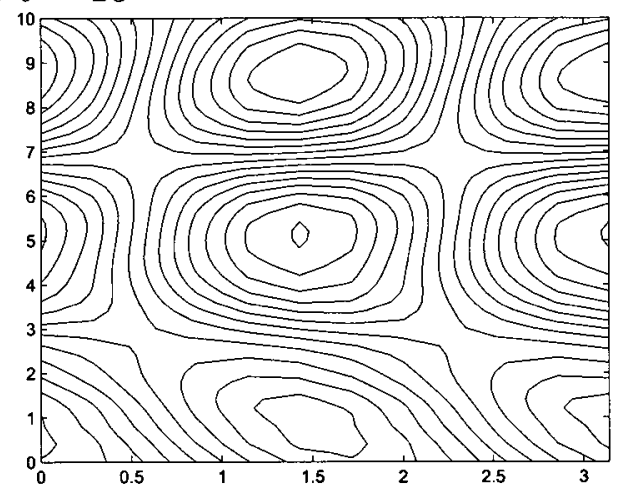

(e) $t=45$

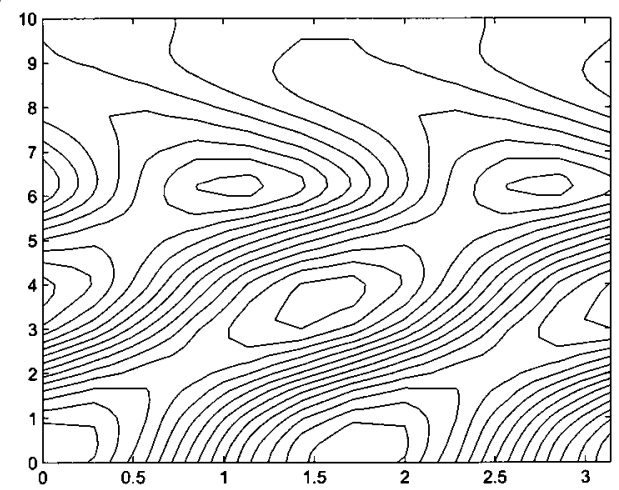

(b) $t=8$

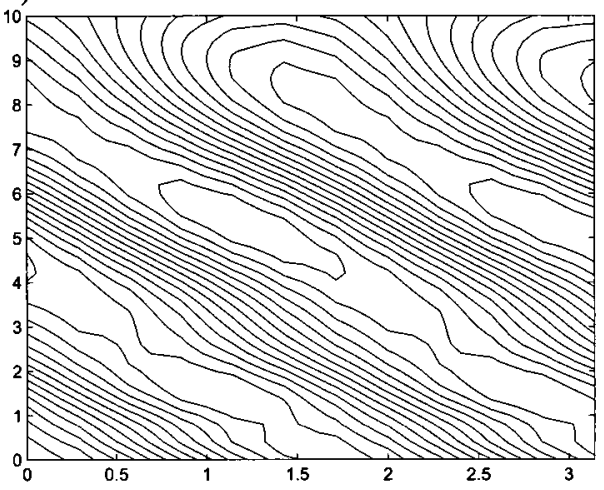

(d) $t=35$

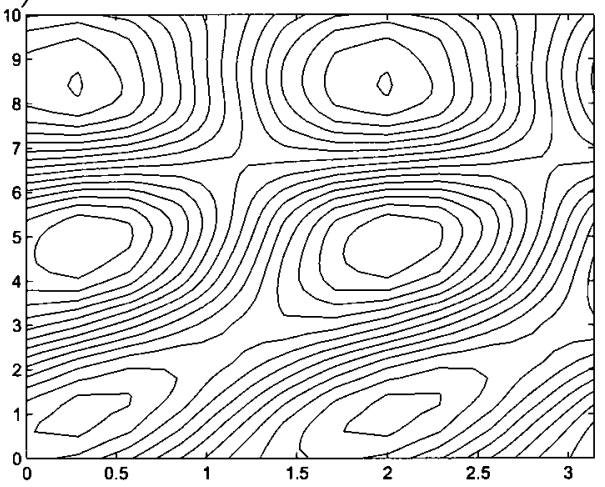

(f) $t=65$

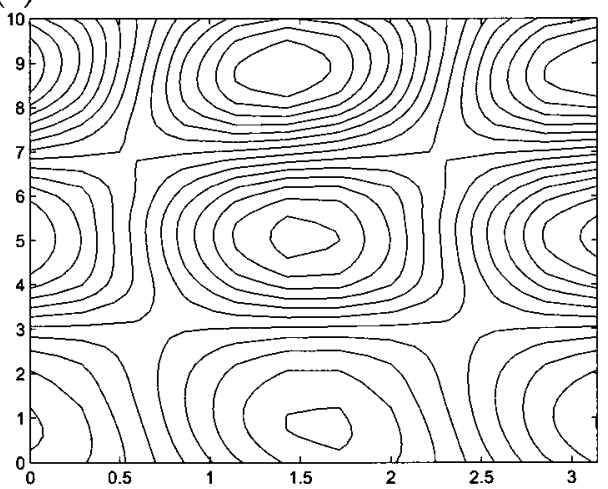

Figure 5.24: Linear simulation of gravity wave propagation $(\varepsilon=0)$ with the timedependent radiation condition: contour plots of the streamfunction $\psi(x, z, t)$ obtained at (a) $t=4$, (b) $t=8$, (c) $t=15$, (d) $t=35$, (e) $t=45$ and (f) $t=65$. A periodic forcing $e^{i k_{o} x}, k_{o}=2$, has been applied at the lower boundary of the rectangular domain and $\delta=0.2$. The background flow is constant, $\bar{u}=1$. 


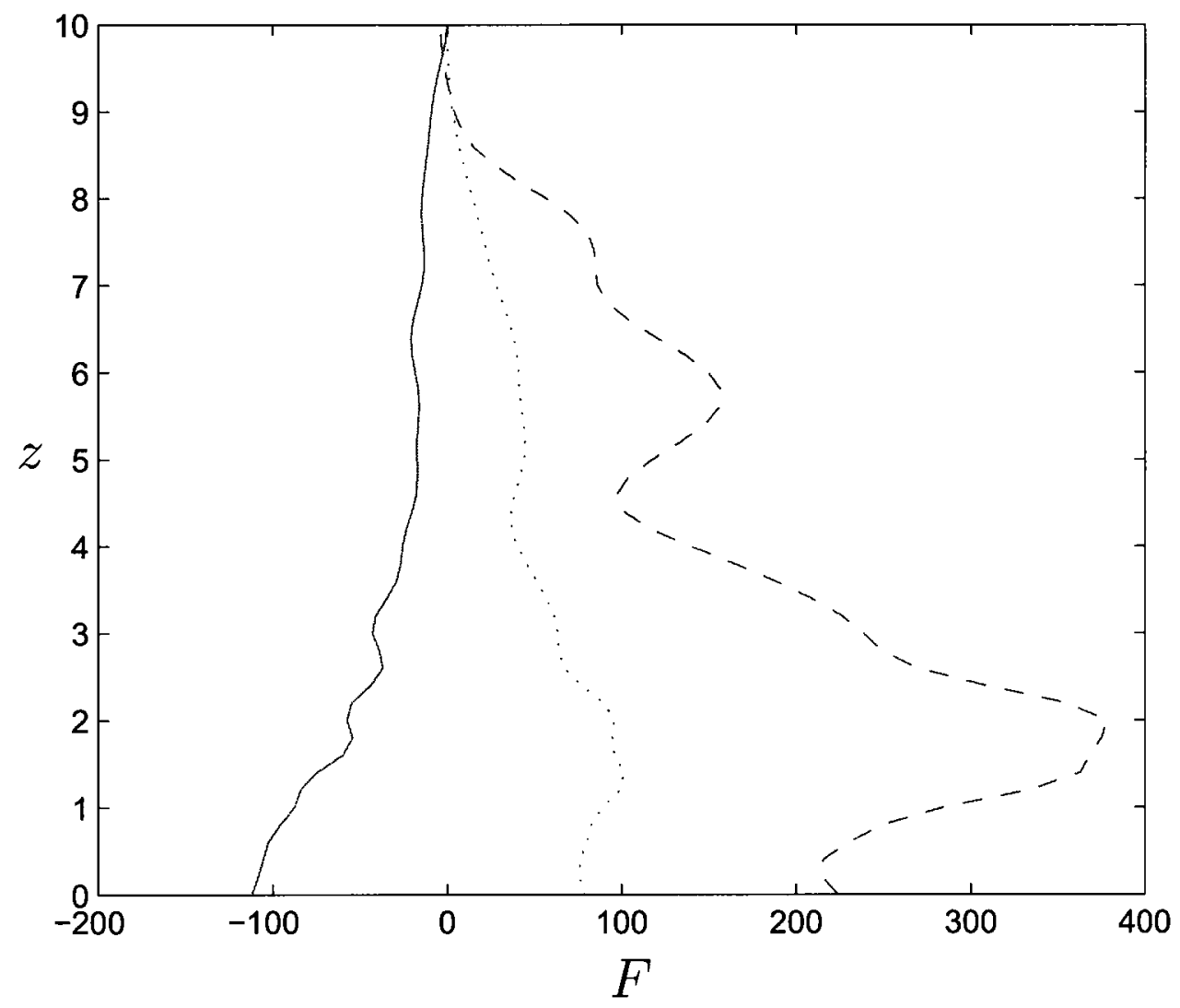

Figure 5.25: Linear simulation of gravity wave propagation $(\varepsilon=0)$ : plot of the averaged vertical momentum flux $F$ as a function of $\mathrm{z}$ at the time $t=40$. A periodic forcing $e^{i k_{o} x}, k_{o}=2$, has been applied at the lower boundary of the rectangular domain and $\delta=0.2$. The background flow is constant, $\bar{u}=1$. The solid line corresponds to the simulation with the time-dependent radiation condition and the dotted line corresponds to the simulation with the zero boundary condition while the dashed line is the result obtained for the simulation with the steady radiation condition. 
(a) $t=4$

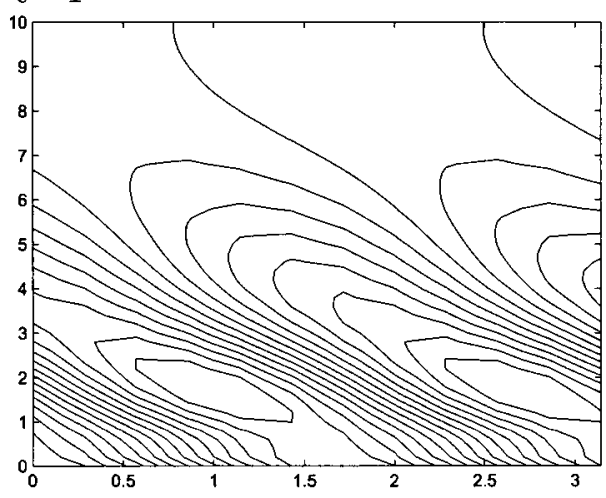

(c) $\mathrm{t}=15$

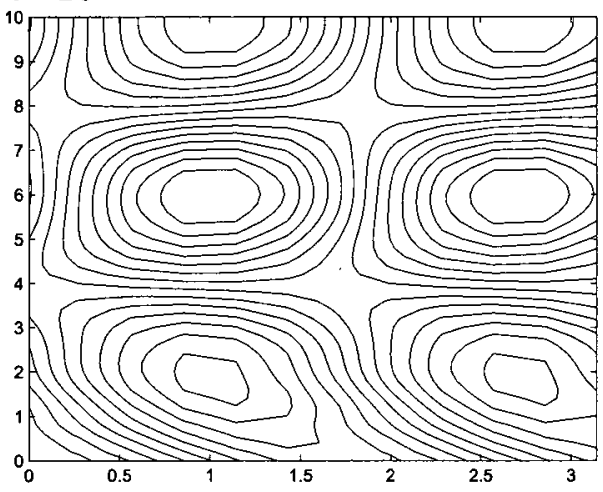

(e) $\mathrm{t}=45$

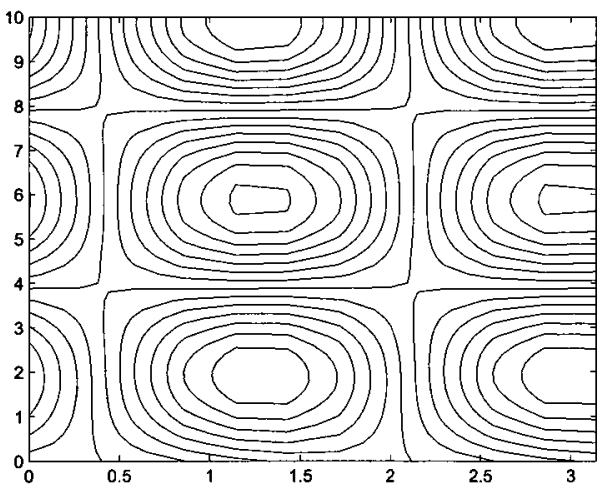

(b) $t=8$

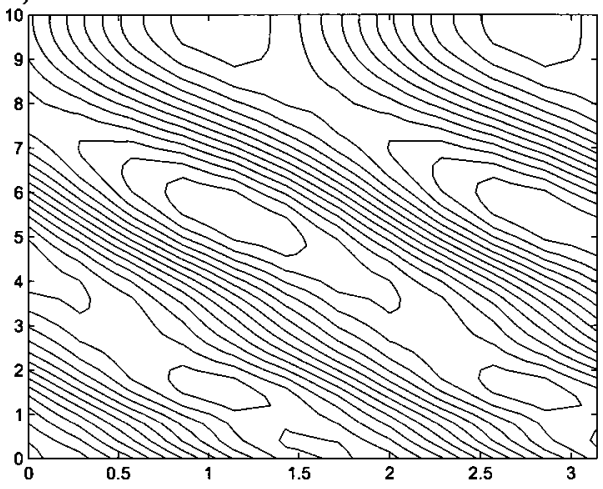

(d) $t=35$

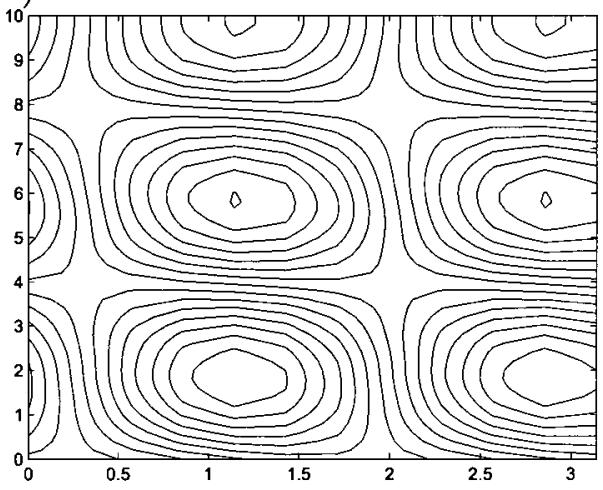

(f) $t=65$

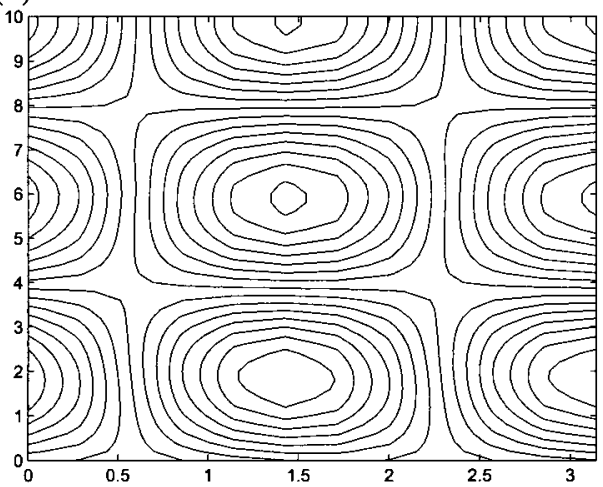

Figure 5.26: Linear simulation of gravity wave propagation $(\varepsilon=0)$ with the time-dependent radiation condition in the long-wave limit $\delta=0$ : contour plots of the streamfunction $\psi(x, z, t)$ obtained at (a) $t=4$, (b) $t=8$, (c) $t=15$, (d) $t=35$, (e) $t=45$ and (f) $t=65$. A periodic forcing $e^{i k_{o} x}, k_{o}=2$, has been applied at the lower boundary of the rectangular domain, $R i=2$. The background flow is constant, $\bar{u}=1$. 


\subsubsection{The results of nonlinear simulations}

Figures 5.27-5.34 show the results from nonlinear simulations of gravity waves propagations. If the zero boundary condition is implemented waves are reflected at the outflow boundary as in the linear problem. The slight differences of the contour plots from the linear simulations in Figure 5.22 and the nonlinear simulations in Figure 5.27 are due to nonlinear waves interactions as in the Rossby wave problem.

Figure 5.28 shows the results of the simulations if the steady radiation condition is implemented at the outflow boundary. In this case the deformations of the contour plots are again observed as in the linear problem. If the simulations are continued at later time the simulations fail at $t=60$ as a result of nonlinear instabilities as seen in Figure 5.28(f).

The results are shown in Figure 5.29 if the time-dependent radiation condition is implemented at the outflow boundary, there are no deformations of the contour plots and the waves evolve with time near the outflow. This is again explained by the fact that by implementing the time-dependent radiation condition we take into account the transient evolution of the waves inside the rectangular domain. These observations suggest that the time-dependent radiation condition gives more realistic results than the other two boundary conditions.

To confirm this, in Figures 5.30-5.32 we compare the 3 sets of results with those obtained using an extended domain $0<z<30$ as we did for the Rossby wave case in section 5.1.3. We observe in Figure 5.30 that of the 3 simulations on the domain $0<z<10$ the one with the time-dependent boundary condition shows the closest agreement with that on an extended domain both with regard to the shape of the contours near the outflow boundary $z=10$ and to the contour 
levels. The graphs of the wave amplitude (Figure 5.31) and the horizontally averaged momentum flux (Figure 5.32) as function of time $0<t<10$ at fixed $z=6$ also support the conclusion that the results obtained with the timedependent boundary condition is the closest to that obtained with the extended domain. 
(a) $t=4$

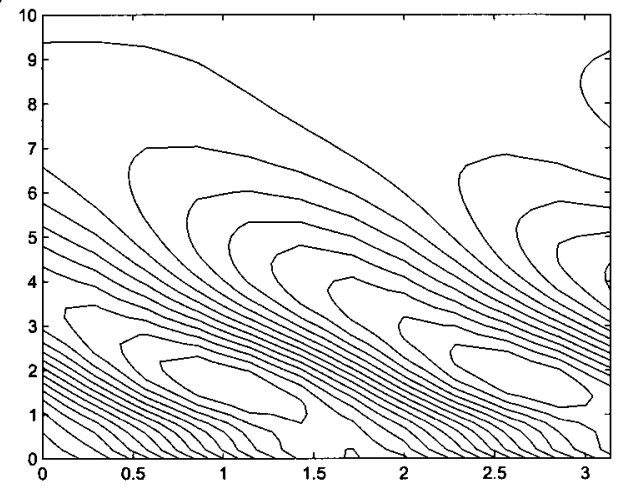

(c) $t=15$

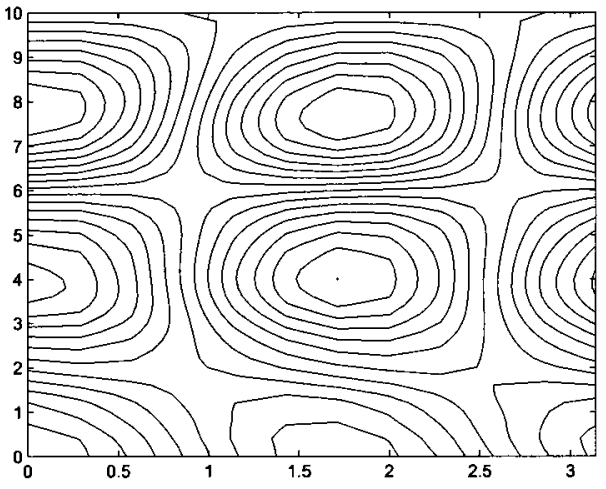

(e) $t=45$

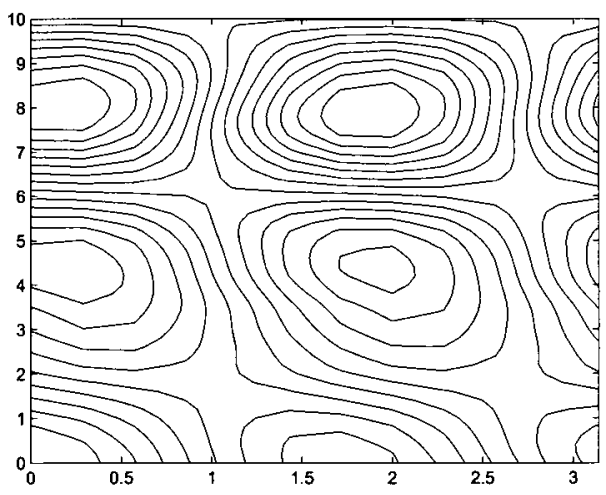

(b) $t=8$

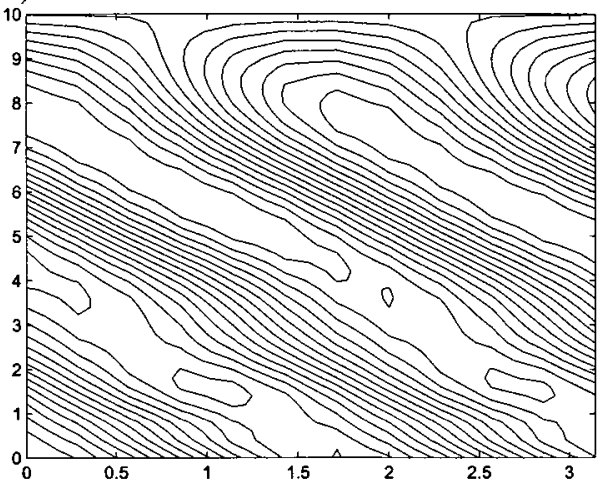

(d) $t=35$

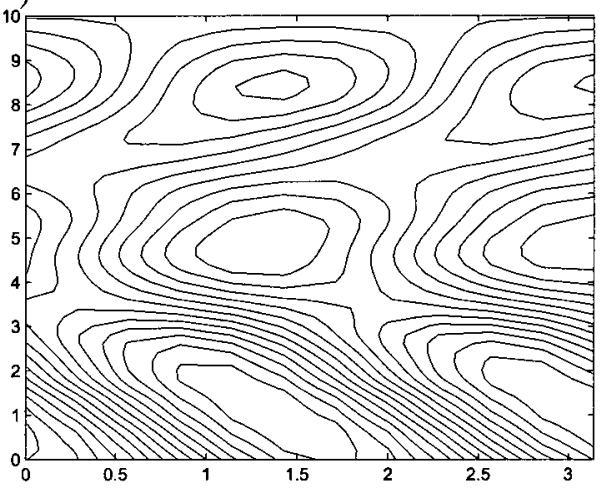

(f) $t=62$

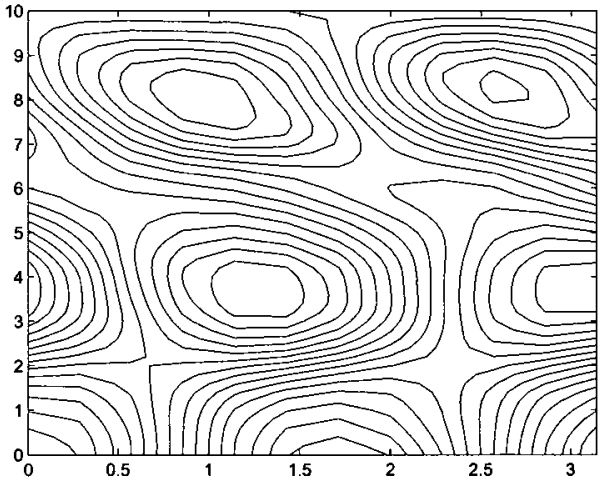

Figure 5.27: Nonlinear simulation of gravity wave propagation $(\varepsilon=0.05)$ with the zero boundary condition: contour plots of the streamfunction $\psi(x, z, t)$ obtained at (a) $t=4$, (b) $t=8$, (c) $t=15$, (d) $t=35$, (e) $t=45$ and (f) $t=62$. A periodic forcing $e^{i k_{o} x}, k_{o}=2$, has been applied at the lower boundary of the rectangular domain and $\delta=0.2$. The background flow is constant, $\bar{u}=1$. 
(a) $t=4$

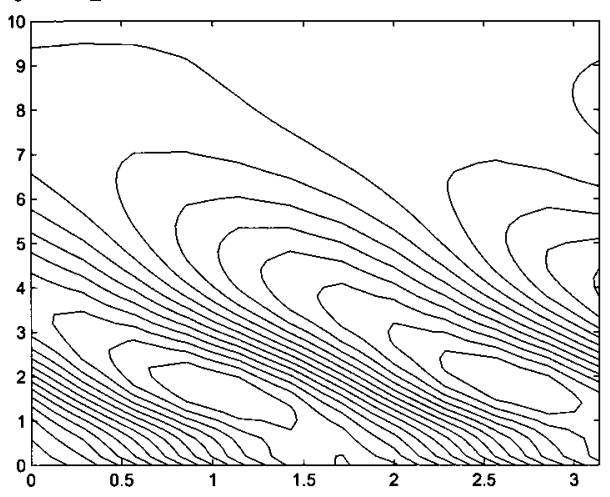

(c) $t=15$

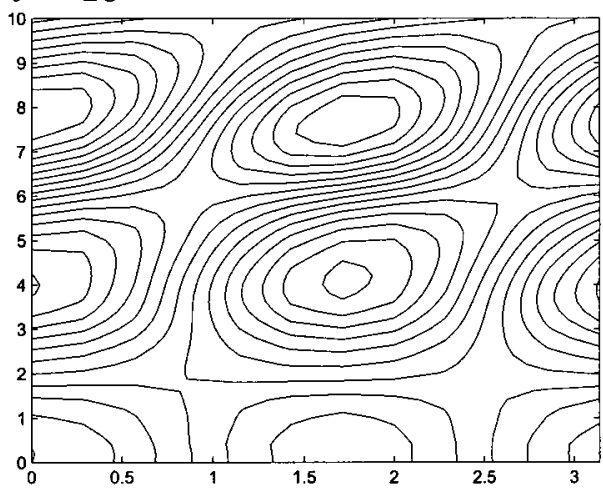

(e) $t=45$

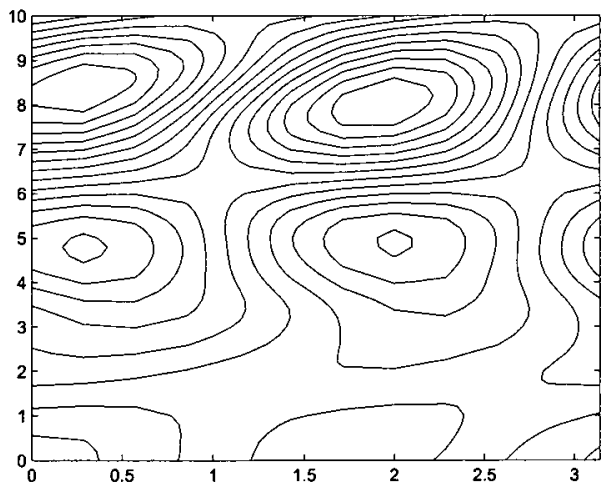

(b) $t=8$

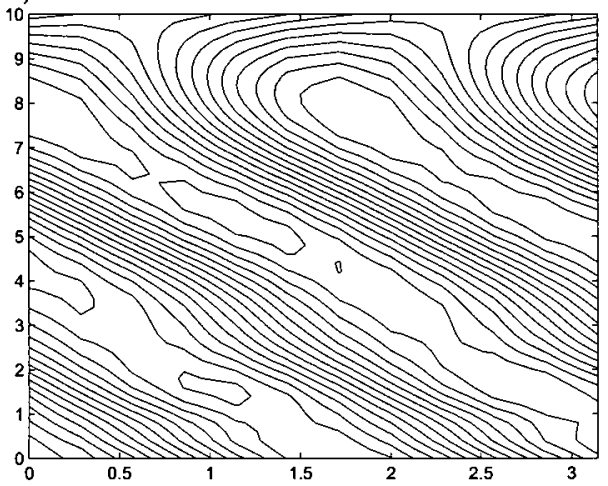

(d) $t=35$

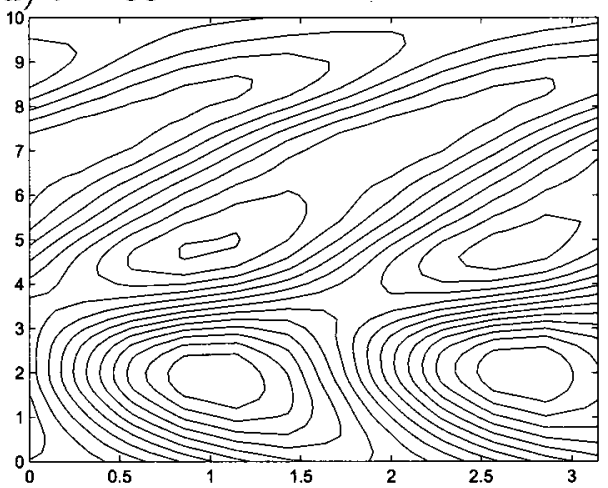

(f) $t=60$

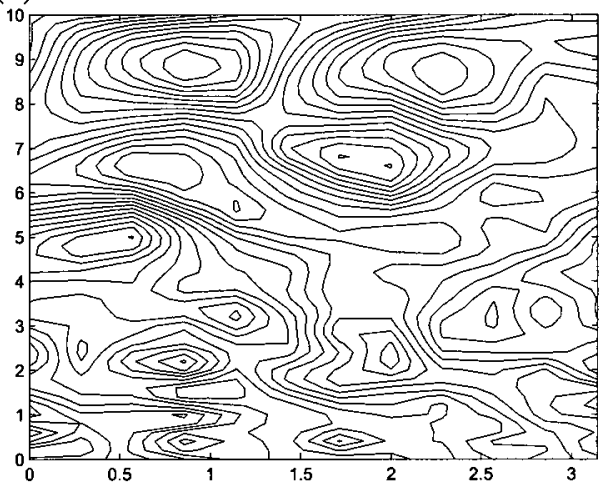

Figure 5.28: Nonlinear simulation of gravity wave propagation $(\varepsilon=0.05)$ with the steady radiation condition: contour plots of the streamfunction $\psi(x, z, t)$ obtained at (a) $t=4$, (b) $t=8$, (c) $t=15$, (d) $t=35$, (e) $t=45$ and (f) $t=60$. A periodic forcing $e^{i k_{o} x}, k_{o}=2$, has been applied at the lower boundary of the rectangular domain and $\delta=0.2$. The background flow is constant, $\bar{u}=1$. 
(a) $t=4$

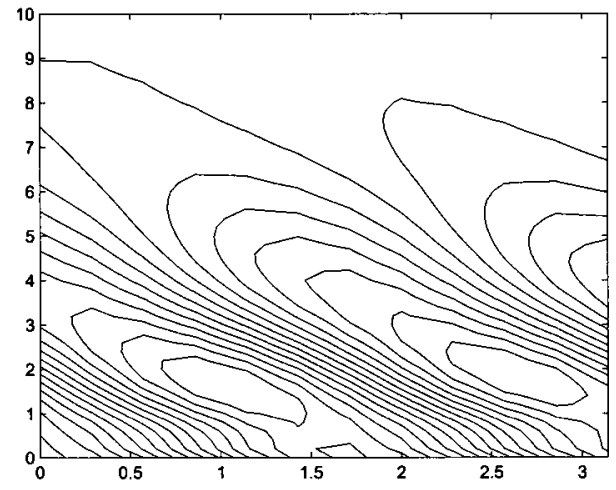

(c) $t=15$

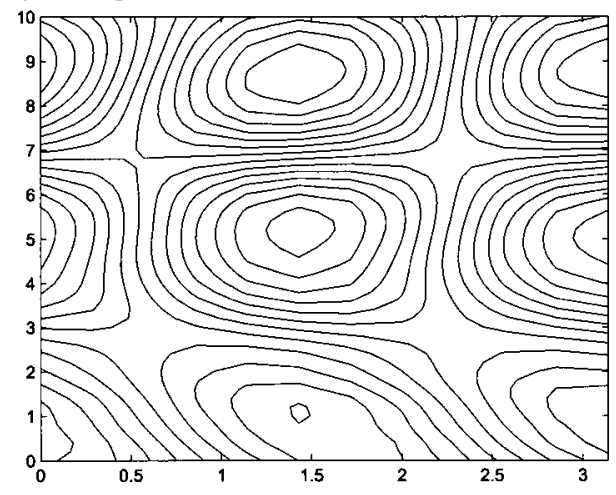

(e) $t=45$

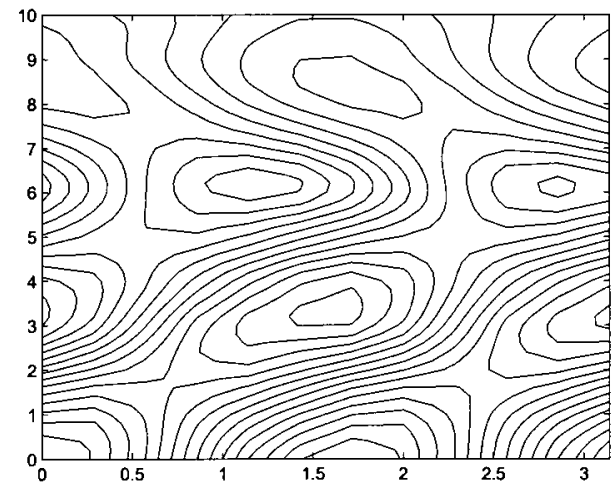

(b) $t=8$

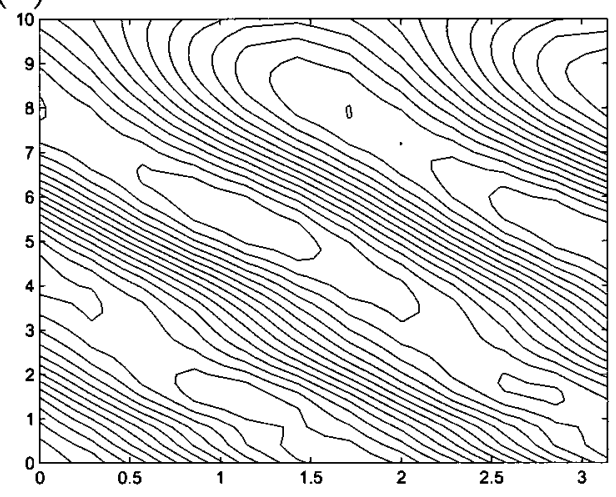

(d) $t=35$

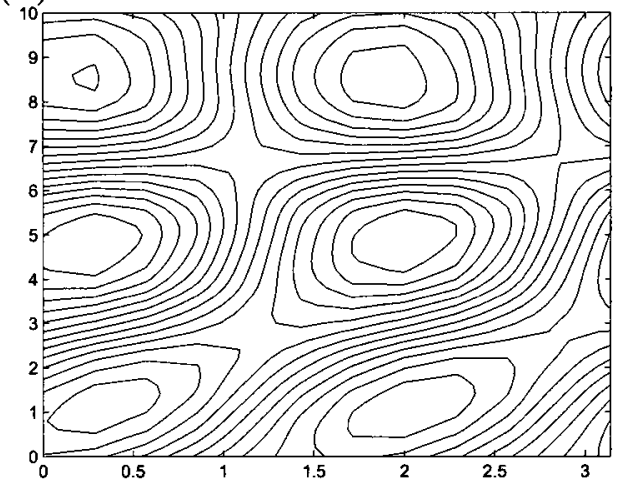

(f) $t=62$

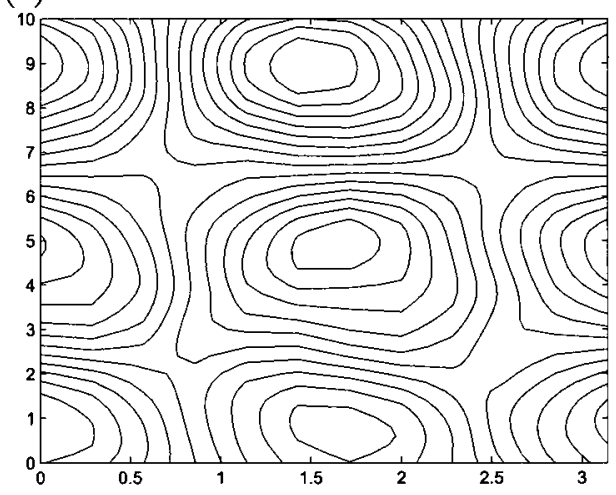

Figure 5.29: Nonlinear simulation of gravity wave propagation $(\varepsilon=0.05)$ with the time-dependent radiation condition: contour plots of the streamfunction $\psi(x, z, t)$ obtained at (a) $t=4$, (b) $t=8$, (c) $t=15$, (d) $t=35$, (e) $t=45$ and (f) $t=62$. A periodic forcing $e^{i k_{o} x}, k_{o}=2$, has been applied at the lower boundary of the rectangular domain and $\delta=0.2$. The background flow is constant, $\vec{u}=1$. 
(a)

(b)

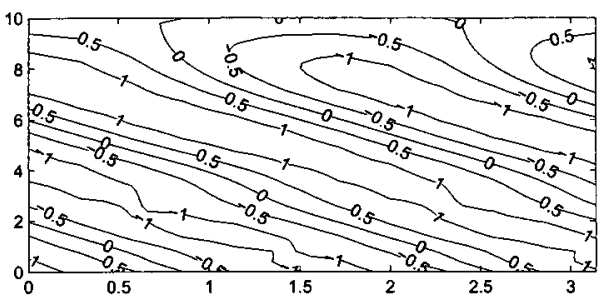

(c)
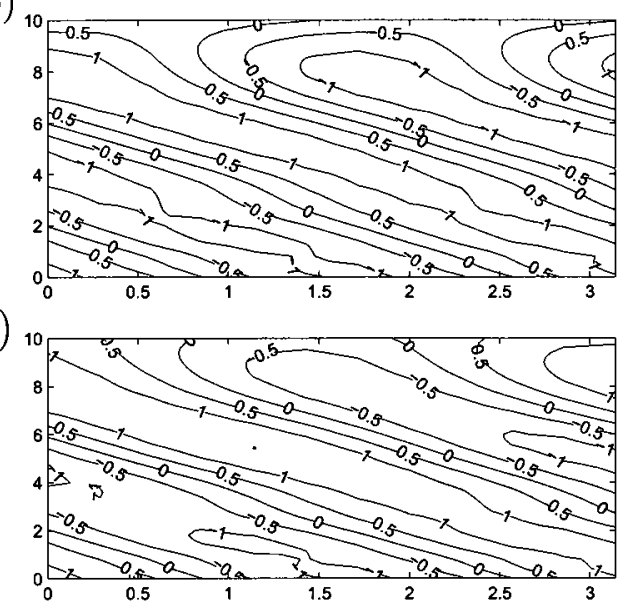

(d)

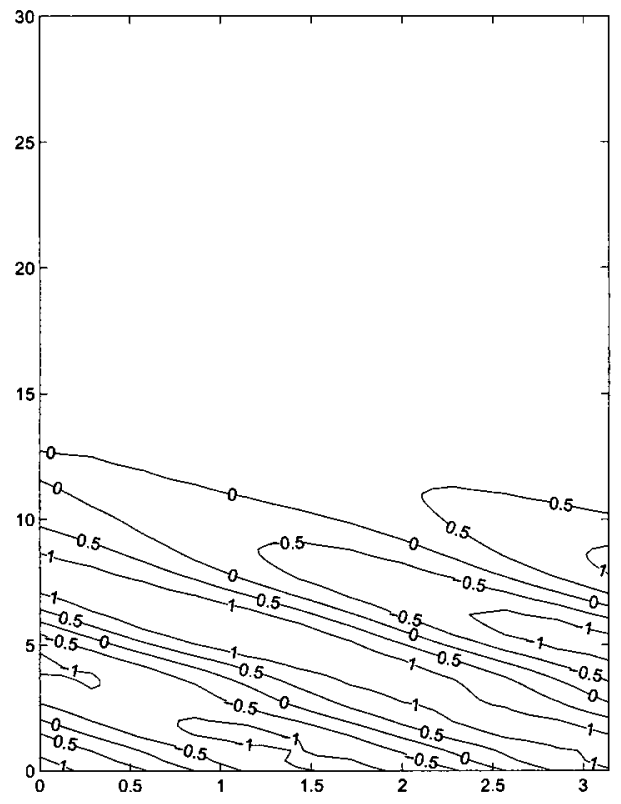

Figure 5.30: Nonlinear simulation of gravity wave propagation $(\varepsilon=0.05)$ : contours plot of the streamfunction $\psi(x, z, t)$ at time $t=8$ with, (a) the zero boundary condition, (b) the steady radiation condition, (c) the time-dependent radiation condition and (d) the zeros boundary condition on a large domain. $\mathrm{A}$ periodic forcing $e^{i k_{o} x}, k_{o}=2$, has been applied at the lower boundary of the rectangular domain and $\delta=0.2$. The background flow is constant, $\bar{u}=1$. 


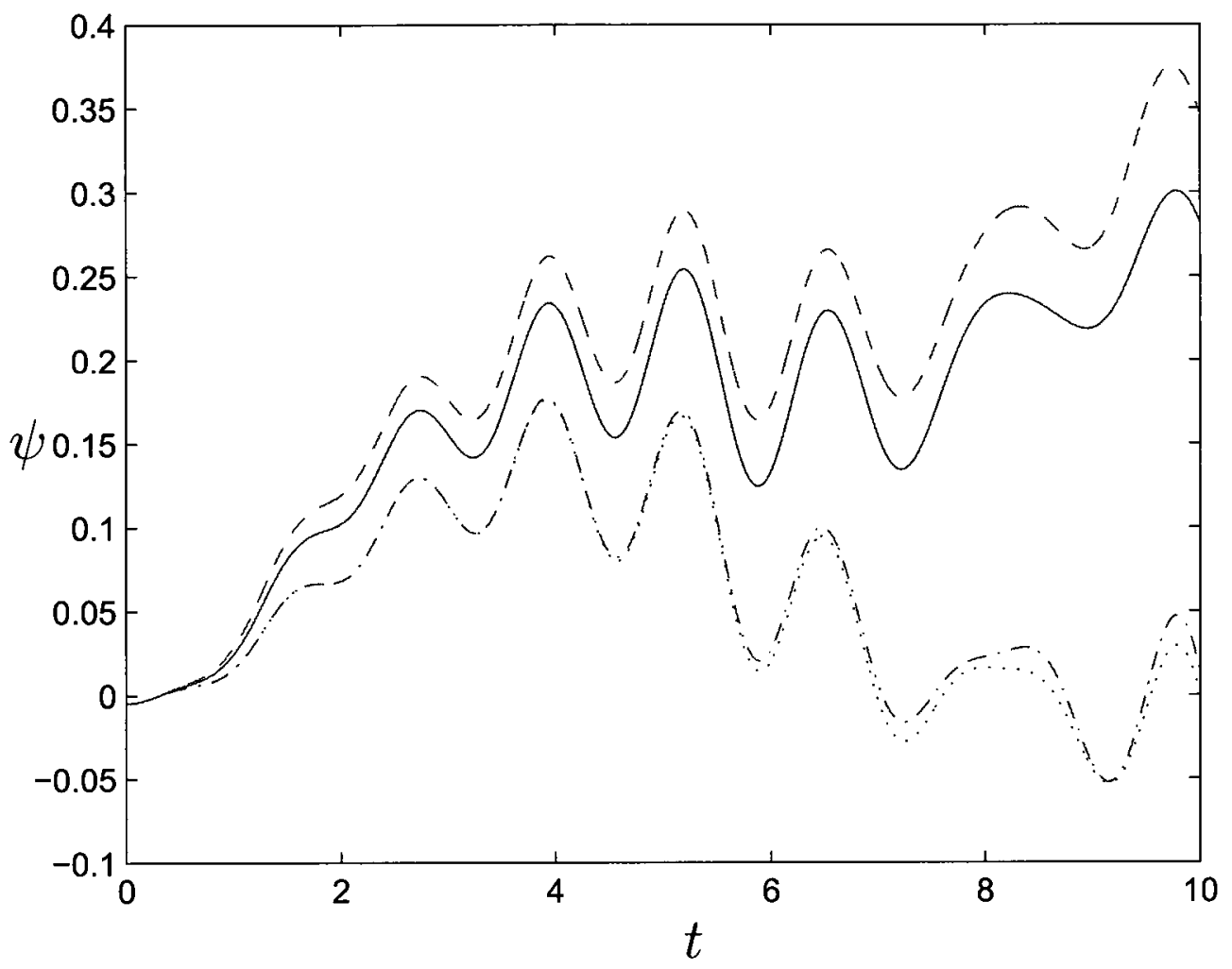

Figure 5.31: Nonlinear simulation of gravity wave propagation $(\varepsilon=0.05)$ : plot of the streamfunction $\psi(x=1.5, z=6, t)$ as a function of time $t, 0<t<10$. A periodic forcing $e^{i k_{o} x}, k_{o}=2$, has been applied at the lower boundary of the rectangular domain and $\delta=0.2$. The background flow is constant, $\bar{u}=1$. The solid line corresponds to the zero boundary condition on a large domain in the $z$-direction, the dashed line corresponds to the time-dependent radiation condition, the dashed-dotted line corresponds to the steady radiation condition and the dotted line to the zero boundary condition. 


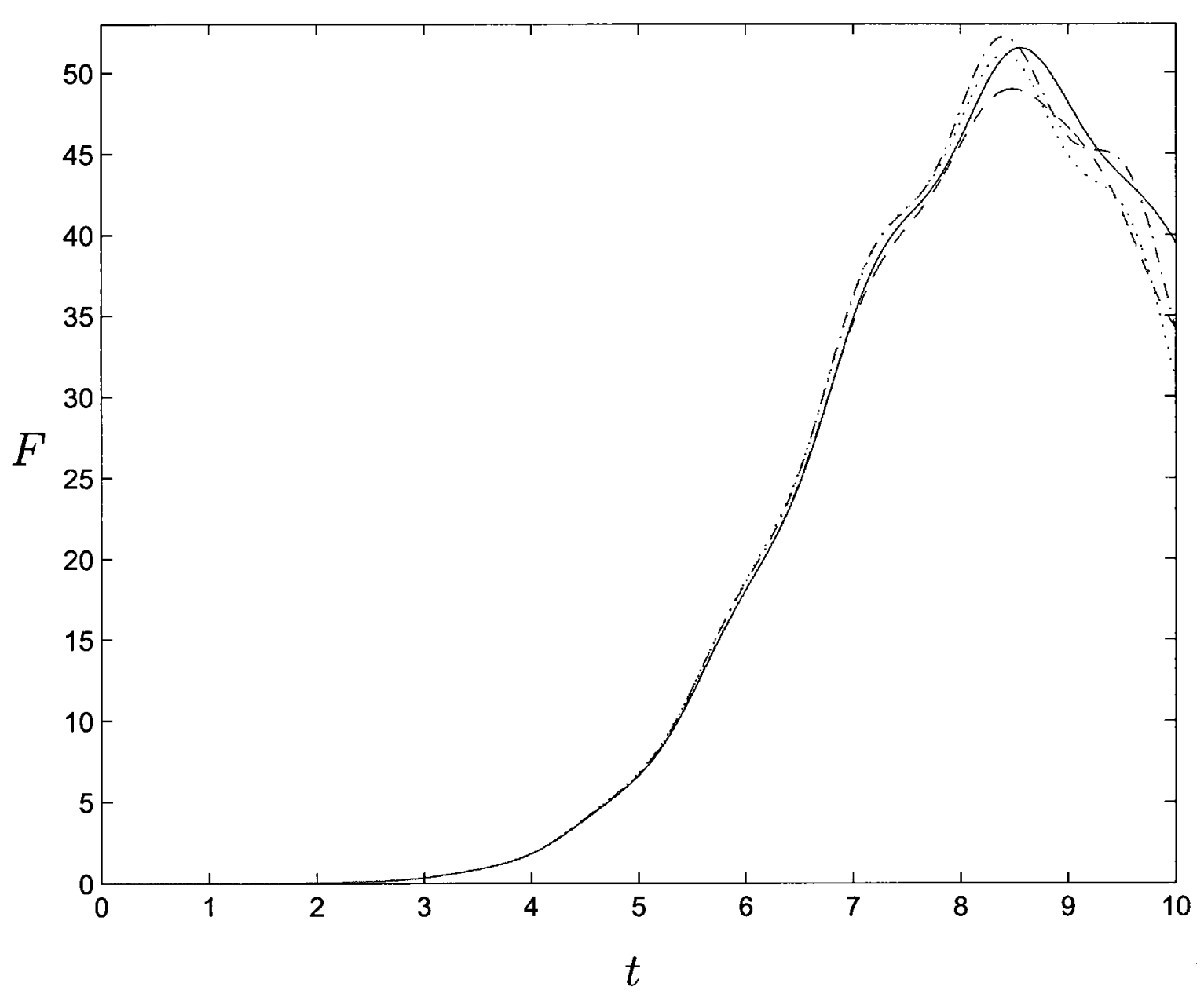

Figure 5.32: Nonlinear simulation of gravity wave propagation $(\varepsilon=0.05)$ : plot of the averaged momentum flux $F(z=6, t)$ as a function of time $t, 0<t<10$. A periodic forcing $e^{i k_{o} x}, k_{o}=2$, has been applied at the lower boundary of the rectangular domain and $\delta=0.2$. The background flow is constant, $\bar{u}=1$. The solid line corresponds to the zero boundary condition on a large domain in the $z$-direction, the dashed line corresponds to the time-dependent radiation condition, the dashed-dotted line corresponds to the steady radiation condition and the dotted line to the zero boundary condition.

Finally we present results obtained by adding a nonlinear correction to the time-dependent radiation condition as described in section 3.3. The nonlinear 
correction (3.76) is added to the zero wavenumber term. The description of the mean flow evolution is given in details in the appendix B. In the linear timedependent radiation condition the contribution of the nonlinearity is omitted and so there is no transfer of the momentum flux at the outflow boundary. By including the nonlinear correction (3.76) we are able to represent the momentum flux transfer at the outflow boundary.

In the Rossby wave problem (Figure 5.18) the zero wavenumber component of the solution was seem to be very small relative to the component of the forced wavenumber. So the effect of the addition of the nonlinear correction to the zero wavenumber which represents the transfer of the momentum flux to the mean flow was negligible.

In the gravity wave problem on the other hand the nonlinear correction is of the same other of magnitude as the components of the forced wavenumber $\kappa= \pm k_{o}$. In fact the dashed line in Figure 5.33 shows that at $t=65$ the nonlinear correction is approximately equal to 11.61 and is in fact large than $|\hat{\psi}|_{\kappa= \pm k_{o}}$ which is approximately to 10 . The solid line shows $|\hat{\psi}|$ computed without the nonlinear correction. In contrast to the Rossby wave case the addition of the nonlinear correction makes a significant difference.

This is further illustrated in Figure 5.34 which shows the wave-induced mean streamfunction $\bar{\psi}_{0}(z, t)$ as a function of $z$ at $t=65$ computed with the nonlinear correction (dashed line) and without the nonlinear correction (solid line). In the gravity wave case there is a larger transfer of the momentum flux from the waves to the mean flow than in the Rossby wave case. This is a characteristic feature of difference between the 2 problems and has been noted by previous researchers (see for example Campbell and Maslowe 2003). 


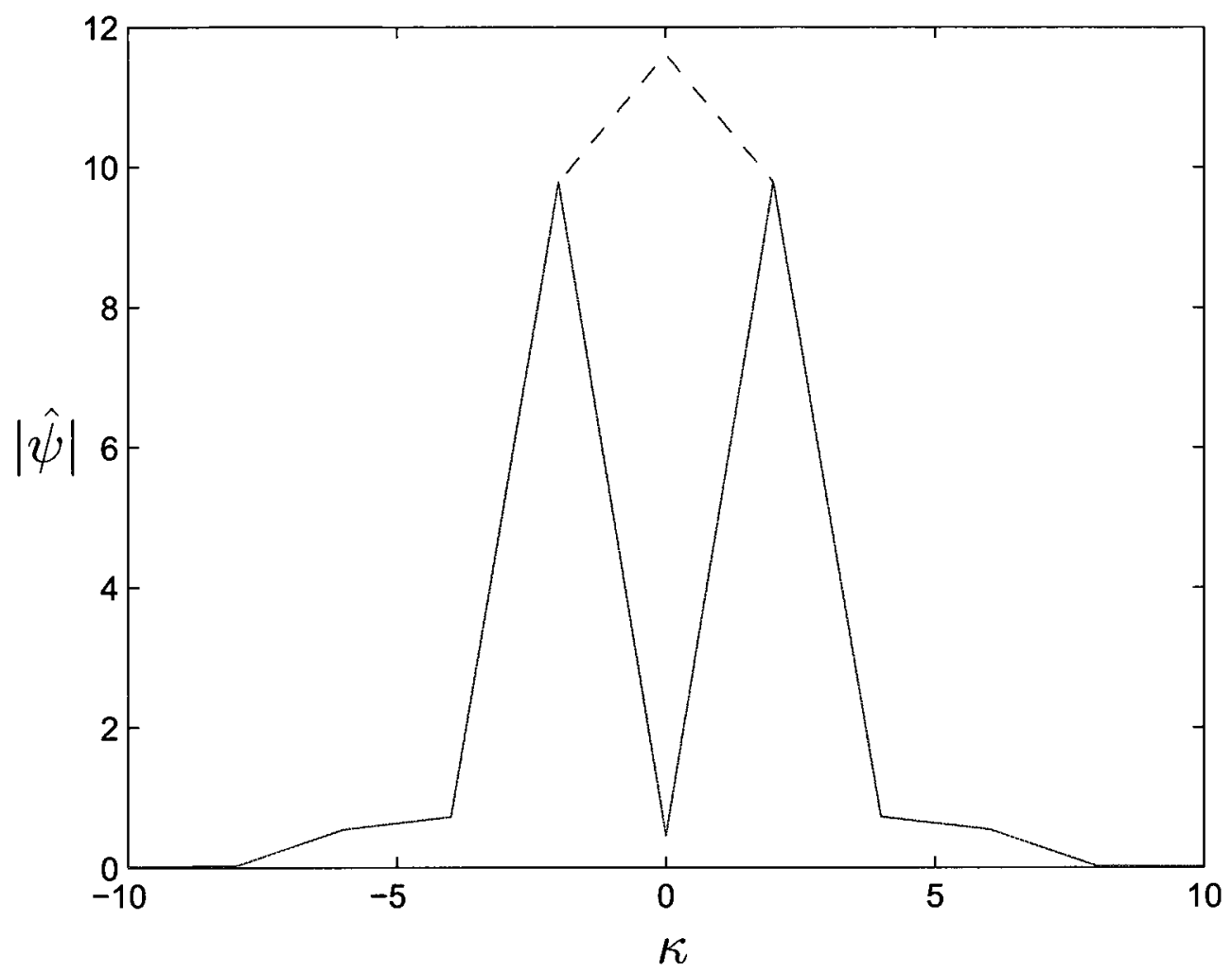

Figure 5.33: Nonlinear simulation of gravity wave propagation $(\varepsilon=0.05)$ : plot of $\hat{\psi}(\kappa, z, t)$ as a function of the wavenumbers $\kappa$ across the outflow boundary at the time $t=65$. A periodic forcing $e^{i k_{o} x}, k_{o}=2$, has been applied at the lower boundary of the rectangular domain and $\delta=0.2$. The background flow is constant, $\bar{u}=1$. The dashed line corresponds to the time-dependent radiation condition with the the nonlinear correction (3.76) while the solid line corresponds to the time-dependent radiation condition without the nonlinear correction. 


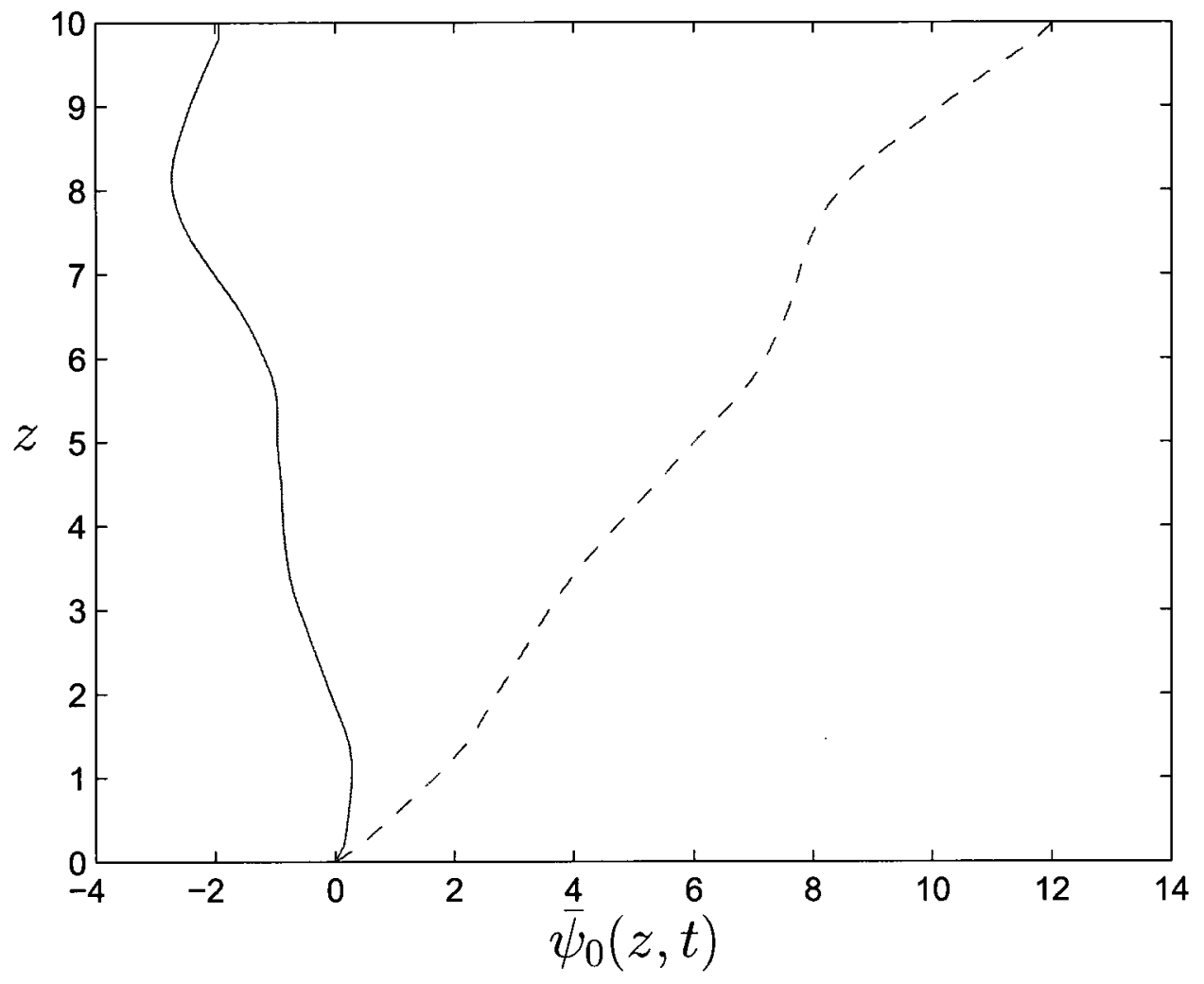

Figure 5.34: Nonlinear simulation of gravity wave propagation $(\varepsilon=0.05)$ : plot of wave-induced mean streamfunction $\bar{\psi}_{0}(z, t)$ as a function of $z$ at the time $t=65$. The dashed line corresponds to the time-dependent radiation condition with the nonlinear correction while the solid line corresponds to the time-dependent radiation condition without the correction. A periodic forcing $e^{i k_{o} x}, k_{o}=2$, has been applied at the lower boundary of the rectangular domain and $\delta=0.2$. The background flow is constant, $\bar{u}=1$. 


\section{Chapter 6}

\section{Conclusions}

In this study different boundary conditions were implemented; a zero boundary condition, a steady radiation condition and a time-dependent radiation condition, in order to simulate the behavior of waves in fluids on an infinite domain using a finite computational domain. We described both linear and nonlinear simulations of Rossby and gravity wave propagation. For both Rossby and gravity waves we have observed that simulations can continue to run and remain stable indefinitely in the linear problem while in the nonlinear problem simulations got to fail at a given finite time.

The correctness of our simulations and results were supported by well knowntheorems, the Miles-Howard theorem for the stability of the flow in the gravity wave problem and the Eliassen-Palm theorem for both Rossby and gravity waves. The correctness of our linear simulations were verified by comparing our results to the exact solutions in the long-wave limit $\delta=0$.

The results of the linear and nonlinear simulations have shown that if the zero boundary condition is implemented at the outflow boundary, the waves are reflected at the outflow boundary for both Rossby and gravity waves and this 
often leads to superpositions of incident and reflected waves.

Since waves are forced to keep the same configuration at the outflow boundary, it was shown that the degradations of the nonlinear simulations are very fast. If the steady boundary condition is implemented at the outflow boundary for both Rossby and gravity waves the waves are forced to remain steady and keep the same configuration at the outflow boundary for all time. This results in increased wave amplitude and instabilities especially in the nonlinear problem.

We observed that simulations can continue to late time without instabilities if the linear time dependent radiation condition is implemented at the out flow boundary. It was seen that if the time-dependent radiation condition is implemented, no variation of wavelength with time is observed inside the rectangular domain like if a zero boundary is implemented, the spatial configuration of the waves are not affected by the behaviors of the waves at the outflow boundary like if the steady radiation condition is applied at the outflow boundary and that the the waves evolve with time near the outflow boundary if the time-dependent radiation condition is implemented at the outflow boundary. This is because the time-dependent radiation condition takes into account the transient evolution of the waves in the computational domain. So the time-dependent radiation condition appears to be the best radiation condition to use in order to simulate the behavior of fluids on an infinite domain. To confirm this we compared ours results with those obtained by extending the computational domain to allow the waves to travel a greater distance before reaching the outflow boundary.

The comparison was carried out for a time frame shorter than the time it would take for the waves to reach the outflow boundary of the extended domain. The results obtained with the time-dependent radiation condition were 
found to be the closest to those obtained with extended domain for both Rossby and gravity waves.

In the special case where the aspect ratio $\delta=0$ the numerical solutions obtained with the time-dependent radiation condition were compared qualitatively with the exact solutions.

We also added a nonlinear correction to the time-dependent radiation condition to represent the transfer of the momentum flux at the outflow boundary.

One of the drawbacks of the time-dependent radiation condition is that it is written in terms of a Laplace convolution integral which is nonlocal in time and expensive to compute requiring the values of the dependent variable at all previous time level. In this study we approximated the convolution integral so as to make the computations local in time and hence less expensive. This will avoid the limitations of using time-dependent radiation conditions because of the computer capacity and hence will make practical the use of time-dependent radiation conditions in geophysical fluid dynamics, for example; numerical weather prediction, general circulation models. 


\section{Appendix A}

\section{Dispersion relation}

In wave mechanics, the dispersion relation is a relation of the form

$$
\omega=\omega(\vec{\Lambda})
$$

where $\omega$ is the pulsation (intrinsic frequency) or the angular frequency, which measures the amount of oscillations that the system undergoes per unit of time independent of the directions or orientations of these oscillations; and $\vec{\Lambda}$ is the wavevector, which gives the direction and displacement or the position of the oscillations. In the Rossby wave case

$$
\vec{\Lambda}=\hat{x} k+\hat{y} l
$$

while in the gravity wave case

$$
\vec{\Lambda}=\hat{x} k+\hat{z} m
$$


The displacement is related to the time by the velocity. This is also true for $\omega$ and $\vec{\Lambda}$. Two types of velocity are very important in wave motion; the phase velocity which is the of propagation of wave and the group velocity which is the velocity of propagation of energy. The group and the phase velocities are respectively given by the vectors

$$
\begin{gathered}
\vec{C}_{g}=\nabla_{\vec{\Lambda}} \omega \\
\vec{C}=\frac{\omega}{\vec{\Lambda}}
\end{gathered}
$$

where $\nabla_{\vec{\Lambda}}$ is the gradient with respect to the wavenumbers while the relation in (A.5) means that $\omega$ is divided by each of the components of $\vec{\Lambda}$. In the Rossby wave case, the group and the phase velocities are given respectively by

$$
\vec{C}_{g}=\hat{x} \frac{\partial \omega}{\partial k}+\hat{y} \frac{\partial \omega}{\partial l}
$$

and

$$
\vec{C}=\hat{x} \frac{\omega}{k}+\hat{y} \frac{\omega}{l}
$$

while in the gravity wave case the group velocity and the phase velocity are given respectively by

$$
\vec{C}_{g}=\hat{x} \frac{\partial \omega}{\partial k}+\hat{z} \frac{\partial \omega}{\partial m}
$$

and

$$
\vec{C}=\hat{x} \frac{\omega}{k}+\hat{z} \frac{\omega}{m}
$$




\section{A.1 Rossby wave dispersion relation}

We use the linearized barotropic vorticity equation (2.51) to derive the dispersion relation for Rossby waves by assuming $\psi$ is the simple plane wave given by

$$
\psi(x, y, t)=A e^{i(k x+l y-\omega t)}
$$

Substituting $\psi$ into equation (2.51) with $\bar{u}=$ constant and rearranging terms yields

$$
(-\omega+\bar{u} k)\left(\delta k^{2}+l^{2}\right)-\beta k=0
$$

Hence $\omega$ is given by

$$
\omega(k, l)=\bar{u} k-\frac{k \beta}{\delta k^{2}+l^{2}}
$$

According to (A.6), the group velocity is given by

$$
\vec{C}_{g}=\hat{x}\left\{\bar{u}-\beta \frac{l^{2}-\delta k^{2}}{\left(\delta k^{2}+l^{2}\right)^{2}}\right\}+\hat{y} \frac{2 \beta l k}{\left(\delta k^{2}+l^{2}\right)^{2}}
$$

while according to (A.7), the phase velocity is given by

$$
\vec{C}=\hat{x}\left\{\bar{u}-\frac{\beta}{\delta k^{2}+l^{2}}\right\}+\hat{y}\left\{\bar{u} \frac{k}{l}-\frac{\beta k}{l\left(\delta k^{2}+l^{2}\right)}\right\}
$$

Thus the phase speed of the Rossby wave relative to the background wind is

$$
C_{x}-\bar{u}=\frac{\beta}{\delta k^{2}+l^{2}}>0
$$

which means that the Rossby wave speed is always easterly (westward) relative to the background wind. 


\section{A.2 Gravity wave dispersion relation}

In order to derive the dispersion relation for gravity waves, consider the simple plane wave

$$
\psi(x, z, t)=A e^{i(k x+m z-\omega t)}
$$

Substituting $\psi$ into equation (2.95) yields

$$
(-\omega+\bar{u} k)^{2}\left(\delta k^{2}+m^{2}\right)+N^{2} k^{2}=0
$$

Solving for $\omega$ yields

$$
\omega(k, m)=\bar{u} k \mp \frac{N k}{\left(\delta k^{2}+m^{2}\right)^{1 / 2}}
$$

According to (A.8), the group velocity is given by

$$
\vec{C}_{g}=\hat{x}\left\{\bar{u} \pm \frac{\left(\delta k^{2}+m^{2}\right)^{1 / 2}-\delta k^{2}}{\left(\delta k^{2}+m^{2}\right)^{3 / 2}}\right\}+\hat{z} \frac{ \pm N k m}{\left(\delta k^{2}+m^{2}\right)^{3 / 2}}
$$

According to (A.9), the phase velocity is given by

$$
\vec{C}=\hat{x}\left\{\bar{u} \mp \frac{N}{\left(\delta k^{2}+m^{2}\right)^{1 / 2}}\right\}+\hat{z}\left\{\bar{u} \frac{k}{m} \mp \frac{N k}{m\left(\delta k^{2}+m^{2}\right)^{1 / 2}}\right\}
$$




\section{Appendix B}

\section{The Eliassen-Palm theorem and the mean flow evolution}

\section{B.1 The nonlinear evolution of the mean flow}

In the nonlinear time-dependent problem given by equation $(2.80)$, due to nonlinear interactions, the mean flow varies with time. In the case of a periodic forcing comprising $e^{i k_{o} x}$ and $e^{-i k_{o} x}$, the perturbation develops higher harmonics corresponding to multiples of $k_{o}$ and $-k_{o}$ and a zero wavenumber terms which corresponds to the mean flow and the mean is given by the average over the zonal wavelength $2 \pi / k_{o}$. In the gravity wave case (and this can be generalized to Rossby waves as well) the perturbation can be written as a Fourier series

$$
\psi(x, z, t)=\sum_{k} \hat{\psi}_{k}(z, t) e^{i k_{o} k(x-c t)}
$$


The streamfunction perturbation can thus be written as

$$
\psi(x, z, t)=\bar{\psi}_{0}(z, t)+\varepsilon \psi_{1}(x, z, t)
$$

where $\bar{\psi}_{0}(z, t)=\varepsilon \hat{\psi}(\kappa=0, z, t)$ is the wave induced mean streamfunction and $\psi_{1}(x, z, t)$ comprises the periodic part of the disturbance corresponding to the wavenumber $\pm k_{o} \pm 2 k_{o} \pm 3 k_{o}, \cdots$. Combining (2.80) and (B.2), and averaging over a zonal wavelength yields

$$
\frac{\partial}{\partial t} \frac{\partial \bar{\psi}_{0}}{\partial z}-\nu \frac{\partial}{\partial z} \frac{\partial^{2} \bar{\psi}_{0}}{\partial z^{2}}+\varepsilon^{2} \frac{k_{o}}{2 \pi} \frac{\partial}{\partial z} \int_{0}^{2 \pi / k_{o}} \psi_{x} \psi_{z} d x=0
$$

The average of the nonlinear term over the wavelength $2 \pi / k_{o}$ is given by

$\overline{J\left(\psi, \nabla^{2} \psi\right)}=\int_{0}^{2 \pi / k_{o}} J\left(\psi, \nabla^{2} \psi\right) d x=\varepsilon \int_{0}^{2 \pi / k_{o}}\left(\psi_{x} \psi_{z z z}+\delta \psi_{x} \psi_{z x x}-\psi_{z} \psi_{z z x}-\delta \psi_{z} \psi_{x x x}\right) d x$

Using integration by parts and the fact that $\psi$ is periodic in $x$, the second term and the fourth term on the right side of (B.5) cancel to yield

$$
\overline{J\left(\psi, \nabla^{2} \psi\right)}=\varepsilon \int_{0}^{2 \pi / k_{o}}\left(\psi_{x} \psi_{z z z}-\psi_{z} \psi_{z z x}\right) d x
$$

Integrating by parts again we obtain

$$
\int_{0}^{2 \pi / k_{o}} \psi_{z} \psi_{z z x} d x=-\int_{0}^{2 \pi / k_{o}} \psi_{x z} \psi_{z z} d x
$$


We add $\psi_{z} \psi_{z z x}$ and $\psi_{x z} \psi_{z z}$ in the integrand in (B.5) to obtain

$$
\begin{aligned}
& \overline{J\left(\psi, \nabla^{2} \psi\right)}=\varepsilon \int_{0}^{2 \pi / k_{o}}\left(\psi_{x} \psi_{z z z}-\psi_{z} \psi_{z z x}+\psi_{z} \psi_{z z x}+\psi_{z x} \psi_{z z}\right) d x \\
= & \varepsilon \int_{0}^{2 \pi / k_{o}}\left(\psi_{x} \psi_{z z z}+2 \psi_{z z} \psi_{z x}+\psi_{z} \psi_{z z x}\right) d x=\varepsilon \frac{\partial^{2}}{\partial z^{2}} \int_{0}^{2 \pi / k_{o}} \psi_{x} \psi_{z} d x
\end{aligned}
$$

So equation (B.3) can be written as

$$
\frac{\partial \bar{u}_{0}}{\partial t}-\nu \frac{\partial^{2} \bar{u}_{0}}{\partial z^{2}}=-\varepsilon^{2} \frac{\partial}{\partial z}(\overline{u w})
$$

which is the usual mean flow evolution equation for gravity waves, and $\bar{u}_{0}(z, t)=$ $-\bar{\psi}_{0 z}(z, t)$. The quantity

$$
F=\overline{u w}=\frac{k_{o}}{2 \pi} \int_{0}^{2 \pi / k_{o}} u w d x=-\frac{2 \pi}{k_{o}} \int_{0}^{2 \pi / k_{o}} \psi_{x} \psi_{z} d x
$$

is called the Reynolds stress (or horizontal momentum flux), the overbar indicates the average has been taken over a wavelength. If the viscosity is neglected equation (B.8) can simply be written as

$$
\frac{\partial \bar{u}_{0}}{\partial t}=-\varepsilon^{2} \frac{\partial F}{\partial z}
$$

The left-hand side of (B.10) can be seen as the rate of change in the mean flow with time as a result of the nonlinear interactions. If the Reynolds stress $F$ is independent of time, the change in the mean flow velocity shall be steady and the mean increases linearly with time. The mean velocity shall be constant also if $F$ is independent of height $z$ as in the Eliassen-Palm theorem (Appendix B.2). 
In the Rossby wave case, the mean flow evolution equation is given by

$$
\frac{\partial \bar{u}_{0}}{\partial t}-\nu \frac{\partial^{2} \bar{u}_{0}}{\partial y^{2}}=-\varepsilon^{2} \frac{\partial}{\partial y}(\overline{u v})
$$

while the Reynolds stress $F$ (or the zonally averaged meridional momentum flux per unit of mass) is given by

$$
F=-\frac{k_{o}}{2 \pi} \int_{0}^{2 \pi / k_{o}} \psi_{x} \psi_{y} d x
$$

If the viscosity is neglected, the mean flow evolution equation is simply given by

$$
\frac{\partial \bar{u}_{0}}{\partial t}=-\varepsilon^{2} \frac{\partial F}{\partial y}
$$

\section{B.2 The Eliassen-Palm theorem}

The Eliassen-Palm theorem (Eliassen and Palm(1961)) usually is applied to gravity waves. Here we state and prove it having in mind that it can be applied for Rossby waves as well.

The Eliassen-Palm theorem:

For linear waves the momentum flux $F$ is independent of height in the absence of singularities.

\section{Proof:}

Let us consider a plane wave

$$
\psi(x, z, t)=A e^{i(k x+m z-\omega t)}+c . c .=A e^{i(k x+m z-\omega t)}+A^{*} e^{-i(k x+m z-\omega t)}
$$


so that

$$
\psi_{x} \psi_{z}=-k m\left(A^{2} e^{i 2(k x+m z-\omega t)}+A^{* 2} e^{-2 i(k x+m z-\omega t)}-2 A A^{*}\right)
$$

Averaging $\psi_{x} \psi_{z}$ over the $x$-wavelength $2 \pi / k_{o}$ yields

$$
F=\overline{\psi_{x} \psi_{z}}=\overline{u w}=-2 k_{o} m|A|^{2}
$$

Hence, $F$ is independent of $z$.

Now consider the more general configuration where the amplitude of the waves depends on $z$ and $t$

$$
\psi(x, z, t)=\phi(z, t) e^{i k x}+c . c .=\phi(z, t) e^{i k x}+\phi^{*}(z, t) e^{-i k x}
$$

Therefore, the vertical flux of horizontal momentum is given by

$$
\begin{array}{r}
\psi_{x} \psi_{z}=\left(i k \phi(z, t) e^{i k x}-i k \phi^{*}(z, t) e^{-i k x}\right)\left(\phi_{z}(z, t) e^{i k x}+\phi_{z}^{*}(z, t) e^{-i k x}\right) \\
=i k \phi \phi_{z} e^{i 2 k x}-i k \phi^{*} \phi_{z}^{*} e^{-i 2 k x}+i k \phi \phi_{z}^{*}-i k \phi^{*} \phi_{z}
\end{array}
$$

Averaging $\psi_{x} \psi_{z}$ over the $x$-wavelength $2 \pi / k_{o}$ yields

$F(z, t)=\overline{\psi_{x} \psi_{z}}=i k \phi(z, t) \phi_{z}^{*}(z, t)-i k \phi^{*}(z, t) \phi_{z}(z, t)=-2 k_{o} \operatorname{Im}\left(\phi^{*}(z, t) \phi_{z}(z, t)\right)$

If we consider the nonlinear problem for which $\psi(x, z, t)$ is given by (B.1), 
the averaged momentum flux will thus be given by

$$
F(z, t)=2 k_{o} \sum_{k=-\infty}^{\infty} \operatorname{Im}\left(k \hat{\psi}_{k}^{*}(z, t) \frac{\partial}{\partial z} \hat{\psi}_{k}(z, t)\right)
$$

In the nonlinear Rossby wave problem given by $(2.50)$, the stream function is given by

$$
\psi(x, y, t)=\sum_{k} \hat{\psi}_{k}(y, t) e^{i k_{o} k(x-c t)},
$$

We can proceed similarly as in the gravity waves case to show the averaged momentum flux is given by

$$
F(y, t)=2 k_{o} \sum_{k=-\infty}^{\infty} \operatorname{Im}\left(k \hat{\psi}_{k}^{*}(y, t) \frac{\partial}{\partial y} \hat{\psi}_{k}(y, t)\right)
$$




\section{Appendix $\mathrm{C}$}

\section{The Richardson number and Miles-Howard theorem}

The Richardson number is a measure of stability of the fluid flow, it is nondimensional and expresses the ratio of the fluid flow potential to the kinetic energy

$$
R i=\frac{g H_{o}}{u^{2}}
$$

where $g$ is the acceleration due to gravity, $H_{o}$ the vertical height scale and $u$ the speed of the fluid flow. For a fluid flow in which the density perturbation is small, e.g. Boussinesq fluid flow, the reduced acceleration $g^{\prime}$ is often used, the relevant parameter is the densimetric Richardson number and is given by

$$
R i=\frac{g^{\prime} H_{o}}{u^{2}}
$$


The reduced acceleration is given by

$$
g^{\prime}=g+a
$$

where $a$ can be calculated using

$$
a=g\left[\frac{R^{2}}{(R+H)^{2}}-1\right]
$$

where $H$ is a given altitude and $R$ the radius of the earth.

For two different types of fluid of density $\rho_{1}$ and $\rho_{2}$ respectively propagating vertically in the opposite directions in the gravitational field, one can use the conservation of linear momentum to obtain

$$
\left(\rho_{1}-\rho_{2}\right) g=g^{\prime} \rho
$$

From (C.5) we have that

$$
g^{\prime}=g \frac{\rho_{1}-\rho_{2}}{\rho}
$$

where in rotating fluid the density of the mixed fluid $\rho$ is approximately equal to the reduced density given by

$$
\frac{1}{\rho}=\frac{1}{\rho_{1}}+\frac{1}{\rho_{2}}
$$

In atmospheric and ocean science the Richardson number has a more general form which takes into account the stratification of the fluid flow

$$
R i=\frac{-\frac{g}{\rho} \frac{\partial \rho}{\partial z}}{\left(\frac{\partial u}{\partial z}\right)^{2}}=\frac{N^{2}}{\left(\frac{\partial u}{\partial z}\right)^{2}}
$$


where $N$ is the Brunt Väisälä frequency. $N^{2}=-\frac{g}{\rho} \frac{\partial \rho}{\partial z}$ measures the extent of stratification while $\frac{\partial u}{\partial z}$ measures the strength of the shear. A condition on stability is that $R i \geq 1 / 4$. This criterion is known as the Miles-Howard theorem.

Miles-Howard theorem(Miles, 1961), (Howard, 1961) :

For stationary solutions of the incompressible Euler equations in the form of parallel flows, a sufficient condition for stability is that the Richardson number be greater than or equal to $1 / 4$ every where in the fluid flow (ref to Warren (1968) for the proof).

Also $R i<0$ if $\rho_{z}>0$ which means the density increases with height. This is known as unstable stratification. In an incompressible, in a static state, the criterion for determining the stability of the medium is that:

- the medium is stable if the density decreases upwards

- the medium is unstable if the density increases upwards

- the medium is neutral if the density is uniform with height 


\section{Appendix D}

\section{Exact solution for transient}

\section{Rossby waves in the long-wave}

\section{limit}

The transient Rossby wave propagation is governed by the inviscid barotropic vorticity equation. For the special case where the the mean flow profile is constant $\bar{u}=$ constant and considering the linearized inviscid barotropic vorticity equation (2.51) and the aspect ratio $\delta=0$ with the forcing $\psi\left(x, y_{1}, t\right)=e^{i k_{o} x}$ at $y=y_{1}$ one can take the Laplace transform in time to derive an exact expression for transient Rossby waves in the long-wave limit $\delta=0$.

In order to do so let us first consider a solution of the form $\psi(x, y, t)=$ $\phi(y, t) e^{i k_{o} x}$. Substituting $\psi(x, y, t)$ in equation (2.51), taking the Laplace transform in time and applying the condition of initial zero vorticity $\phi_{y y}(y, 0)$ yields

$$
\tilde{\phi}_{y y}(y, s)+\left(\frac{i k_{o} \beta}{s+i k \bar{u}}\right) \tilde{\phi}(y, s)=0
$$


where $\tilde{\phi}(y, s)$ is the Laplace transform of $\phi(y, t)$. The solution of the equation (D.1) with the monochromatic forcing at $y=y_{1}$ for downward propagation is given by

$$
\tilde{\phi}(y, s)=\frac{1}{s} e^{i \sqrt{\left(\frac{i k_{o} \beta}{s+i k_{o} \bar{u}}\right)}\left(y-y_{1}\right)}
$$

$\phi(y, t)$ is obtained by inverting the Laplace transform to obtain

$$
\phi(y, t)=\mathcal{L}^{-1}\{\tilde{\phi}(y, s)\}=e^{-i k \bar{u} t} \frac{1}{2 \pi i} \int_{-i \infty}^{i \infty} \frac{1}{s-i k \bar{u}} e^{a s^{-\frac{1}{2}}} e^{s t} d s
$$

after shifting, with $a(y)=i\left(i k_{o} \beta\right)^{\frac{1}{2}}\left(y-y_{1}\right), a$ being a complex function.

To evaluate this integral we integrate along a straight vertical line from $s=$ $\alpha-i R$ to $s=\alpha+i R$ in the complex $s$ plane and then take the limit as $R \rightarrow \infty$. We require that $\alpha>0$ so that the vertical line lies to the right of the singularities of the integrand $s=0$ and $s=i k_{o} \bar{u}$ and then we close the contour around the pole $s=i k_{o} \bar{u}$ and indent it around the branch point $s=0$. In the limit as $R \rightarrow \infty$ the integral along the curved part of the closed contour goes to zero and the integral (D.3) is the sum of the contribution from the pole $s=i k_{o} \bar{u}$ and the branch point $s=0$ according to Cauchy's theorem. This gives the standard Bromwich contour that is used for evaluating inverse Laplace transforms (see for example Ablowitz and Fokas 2003).

The contribution from the pole $s=i k_{o} \bar{u}$ gives the steady part of the solution and is found by computing the residue,

$$
\phi_{o}(y)=e^{-i k_{o} \bar{u} t} \operatorname{Res}\left(s=i k_{o} \bar{u}\right)=e^{-i k_{o} \bar{u} t} e^{i k_{o} \bar{u} t} e^{a\left(\sqrt{i k_{o} \bar{u}}\right)^{-1}}=e^{i \sqrt{\frac{\beta}{\bar{u}}}\left(y-y_{1}\right)}
$$

To evaluate the time-dependent part of the solution, we make the substitution 
$p=+\sqrt{s}$ and integrate from $p=\alpha+e^{-i \pi / 4} R$ to $p=\alpha+e^{i \pi / 4} R$, with $\alpha>0$ and let $R \rightarrow \infty$. The integral becomes

$$
\begin{aligned}
\phi(y, t)= & \mathcal{L}^{-1}\{\tilde{\phi}(y, s)\}=2 e^{-i k \bar{u} t} \frac{1}{2 \pi i} \int_{\alpha+e^{-i \pi / 4} R}^{\alpha+e^{i \pi / 4} R} \frac{1}{p^{2}-i k \bar{u}} e^{a p^{-1}} e^{p^{2} t} p d p \\
& =2 e^{-i k \bar{u} t} \frac{1}{2 \pi i} \int_{\alpha+e^{-i \pi / 4} R}^{\alpha+e^{i \pi / 4} R} \frac{1}{(p-\sqrt{i k \bar{u}})(p+\sqrt{i k \bar{u}})} e^{a p^{-1}} e^{p^{2} t} p d p
\end{aligned}
$$

with $R \rightarrow \infty$.

We close the contour around the singularities $p=0$ and $p=+\sqrt{i k_{o}} \overline{\bar{u}}$. The integral evaluated along the curved part of the contour goes to zero as $R \rightarrow \infty$. The contribution from the pole $p=+\sqrt{i k_{o} \bar{u}}$ gives the steady part of the solution (D.4). The transient part of the solution is given by the contribution from the pole $p=0$ and is

$$
\phi_{1}(y, t)=2 e^{-i k_{o} \bar{u} t} \mathcal{R} e s(p=0)
$$

Before evaluating the integral (D.5) we first consider the integral of $e^{p^{2} t}\left(e^{a p^{-1}}-1\right) p$. This can be expanded out as a Laurent series to yield

$$
e^{p^{2} t}\left(e^{a p^{-1}}-1\right) p=p\left(1+\frac{p^{2} t}{1 !}+\frac{p^{4} t^{2}}{2 !}+. .\right)\left(\frac{a}{p}+\frac{a^{2}}{2 ! p^{2}}+\frac{a^{3}}{3 ! p^{3}}+\ldots\right)
$$

Then we sum up the coefficients corresponding to $p^{-1}$ according to the Residue Theorem. So the integral of $e^{p^{2} t}\left(e^{a p^{-1}}-1\right) p$ is given by the residue at $p=0$ and is 


$$
\begin{aligned}
& \quad \int_{\alpha+e^{-i \pi / 4} R}^{\alpha+e^{i \pi / 4} R}\left(e^{a p^{-1}}-1\right) e^{p^{2} t} p d p=2 \mathcal{R} e s\left\{e^{p^{2} t} e^{a p^{-1}} p\right\}(p=0) \\
& =2 \pi i \sum_{j=0}^{\infty} \frac{a^{2 j+2} t^{j}}{(2 j+2) ! j !}=2 \pi i \sum_{j=0}^{\infty} \frac{\left(-i k_{o} \beta\right)^{j+1}\left(y-y_{1}\right)^{2 j+2} t^{j}}{(2 j+2) ! j !}
\end{aligned}
$$

Let us define

$$
\begin{array}{r}
f_{0}(y, t)=\mathcal{L}^{-1}\left\{e^{a(y) s^{-1 / 2}}-1\right\}=\frac{1}{2 \pi i} \int_{\alpha+e^{-i \pi / 4} R}^{\alpha+e^{i \pi / 4} R} 2\left(e^{a p^{-1}}-1\right) e^{p^{2} t} p d p \\
=2 \pi i \mathcal{R} e s\left\{e^{p^{2} t}\left(e^{a p^{-1}}-1\right) p\right\}(p=0)=2 \sum_{j=0}^{\infty} \frac{a^{2 j+2} t^{j}}{(2 j+2) ! j !} \\
=2 \sum_{j=0}^{\infty} \frac{\left(-i k_{o} \beta\right)^{j+1}\left(y-y_{1}\right)^{2 j+2} t^{j}}{(2 j+2) ! j !}
\end{array}
$$

The Laurent series of $\frac{1}{p^{2}-i k_{o} \bar{u}}$ is

$$
\frac{1}{p^{2}-i k_{o} \bar{u}}=-\frac{1}{i k_{o} \bar{u}} \frac{1}{1-\frac{p^{2}}{i k_{o} \bar{u}}}=-\frac{1}{i k_{o} \bar{u}}\left(1+\frac{p^{2}}{i k_{o} \bar{u}}+\frac{p^{4}}{\left(i k_{o} \bar{u}\right)^{2}}+\frac{p^{6}}{\left(i k_{o} \bar{u}\right)^{3}}+\ldots\right)
$$

So to compute (D.5) we multiply (D.7) and (D.10) again we sum up the coefficients corresponding to $p^{-1}$ according to the Residue Theorem. We find that 
the residue at the pole $p=0$ is given by

$$
\begin{array}{r}
\mathcal{R e s}(p=0)=-\sum_{j=0}^{\infty} \frac{a^{2 j+2}}{\left(i k_{o} \bar{u}\right)^{j+1}(2 j+2) !} \frac{t^{j}}{j !}-\sum_{j=0}^{\infty} \frac{a^{2 j+4}}{\left(i k_{o} \bar{u}\right)^{j+1}(2 j+4) !} \frac{t^{j}}{j !} \\
-\sum_{j=0}^{\infty} \frac{a^{2 j+6}}{\left(i k_{o} \bar{u}\right)^{j+1}(2 j+6) ! j !}-\ldots .
\end{array}
$$

This can be written as a double sum in the form

$$
\mathcal{R} e s(p=0)=-\sum_{l=0}^{\infty} \sum_{j=0}^{\infty} \frac{a^{2 j+2 l+2}}{\left(i k_{o} \bar{u}\right)^{l+1}(2 j+2 l+2) !} \frac{t^{j}}{j !}
$$

The transient part of the solution $\phi_{1}(y, t)$ is thus given by

$$
\phi_{1}(y, t)=-2 \sum_{l=0}^{\infty} \sum_{j=0}^{\infty} \frac{a^{2 j+2 l+2}}{\left(i k_{o} \bar{u}\right)^{l+1}(2 j+2 l+2) !} \frac{t^{j}}{j !}
$$

So the amplitude $\phi(y, t)$ of the downward propagatin is given by

$$
\begin{array}{r}
\phi(y, t)=-2 e^{-i k_{o} \bar{u} t} \sum_{l=0}^{\infty} \sum_{j=0}^{\infty} \frac{1}{\left(i k_{o} \bar{u}\right)^{l+1}} \frac{\left(-i k_{o} \beta\right)^{l+j+1}\left(y-y_{1}\right)^{2 l+2 j+2} t^{j}}{(2 l+2 j+2) ! j !} \\
+e^{-i \sqrt{\frac{\rho}{\bar{u}}}\left(y-y_{1}\right)}=\phi_{0}(y)+\phi_{1}(y, t)
\end{array}
$$

Hence the exact expression for the transient Rossby wave is given by

$$
\begin{array}{r}
\psi(x, y, t)=-2 e^{i k_{o}(x-\bar{u} t)} \sum_{l=0}^{\infty} \sum_{j=0}^{\infty} \frac{1}{\left(i k_{o} \bar{u}\right)^{l+1}} \frac{\left(-i k_{o} \beta\right)^{l+j+1}\left(y-y_{1}\right)^{2 l+2 j+2} t^{j}}{(2 l+2 j+2) ! j !} \\
+e^{i k_{o} x} e^{-i \sqrt{\frac{\beta}{\bar{u}}}\left(y-y_{1}\right)}
\end{array}
$$

An alternative procedure for evaluating $\phi(y, t)$ is to make use of the function 
$f_{0}(y, t)$ given in (D.9) and apply the convolution theorem for Laplace transforms. This implies that the amplitude of the downward propagating wave $\phi(y, t)$ is given by

$\phi(y, t)=\mathcal{L}^{-1}\left\{\frac{e^{a(y)\left(s+i k_{o} \bar{u}\right)^{-1 / 2}}}{s}\right\}=e^{-i k \bar{u} t} \mathcal{L}^{-1}\left\{\frac{1}{s-i k_{o} \bar{u}}\right\} * \mathcal{L}^{-1}\left\{e^{a(y) s^{-\frac{1}{2}}}-1\right\}$

Then

$$
\begin{array}{r}
\phi(y, t)=e^{-i k \bar{u} t} \int_{0}^{t} e^{-i k \bar{u}(\tau-t)}\left(f_{0}(y, \tau)+2 \delta(t-0)\right) d \tau \\
=\int_{0}^{t} e^{-i k \bar{u} \tau}\left(f_{0}(y, \tau)+2 \delta(t-0)\right) d \tau
\end{array}
$$

where

$$
f_{0}(y, t)=\mathcal{L}^{-1}\left\{e^{a s^{-\frac{1}{2}}}-1\right\}
$$

is given by (D.9). The upper limit of the integration gives the time-dependent part $\phi_{1}(y, t)$ and the lower limit gives the steady part of the solution $\phi_{0}(y)$. Repeated integration by parts gives the desired result (D.14).

\section{D.1 Convergence of the solution}

The steady part $\phi_{0}(y)$ is bounded since it is periodic and oscillates between -1 and 1 . We can make use of the the ratio and comparison tests to verify that the 
transient part of the solution $\phi_{1}(y, t)$ is convergent.

To show that $\phi_{1}(y, t)$ is convergent we first consider the series

$$
f_{0}(y, t)=2 \sum_{j=0}^{\infty} \frac{\left(-i k_{o} \beta\right)^{j+1}\left(y-y_{1}\right)^{2 j+2} t^{j}}{(2 j+2) ! j !}
$$

Applying the ratio test to this series, we have that

$$
\frac{a_{j+1}}{a_{j}}=\left(-i k_{o} \beta\right)\left(y-y_{1}\right)^{2} t \frac{(2 j+2) !}{(2 j+4) !}=\left(-i k_{o} \beta\right)\left(y-y_{1}\right)^{2} \frac{t}{(2 j+4)(2 j+3)}(
$$

For finite values of $y$ and $t$ we have that

$$
\lim _{l \rightarrow \infty}\left|\frac{a_{j+1}}{a_{j}}\right|=0
$$

Hence $f_{0}(y, t)$ is convergent. And for each $l=1,2,3,4, \ldots$ we can show that

$$
f_{l}(y, t)=2 \sum_{j=0}^{\infty} \frac{1}{\left(i k_{o} \bar{u}\right)^{l+1}} \frac{\left(-i k_{o} \beta\right)^{l+j+1}\left(y-y_{1}\right)^{2 l+2 j+2} t^{j}}{(2 j+2 l+2) ! j !}
$$

is convergent.

In fact the series $f_{l}(y, t)$ have similar properties to the Bessel functions $\mathcal{J}_{2 l+2}$. To see this let us write $f_{l}(y, t)$ as

$$
f_{l}(y, t)=2 \sum_{j=0}^{\infty} \frac{1}{\left(i k_{o} \bar{u}\right)^{l+1}} \frac{\left(-i k_{o} \beta\right)^{l+j+1}\left(y-y_{1}\right)^{2 l+2 j+2} t^{j}}{(2 j+2 l+2) ! j !}=\frac{g_{l}(y, t)}{t^{l+1}}
$$

so that

$$
f_{l}(y, t)=\frac{2}{\left(i k_{o} \bar{u}\right)^{l+1}}\left(\frac{1}{t}\right)^{l+1} \sum_{j=0}^{\infty} \frac{\left(-i k_{o} \beta\right)^{l+j+1}\left(y-y_{1}\right)^{2 l+2 j+2}(\sqrt{t})^{2 j+2 l+2}}{(2 j+2 l+2) ! j !}
$$


where

$$
g_{l}(y, t)=\frac{2}{\left(i k_{o} \bar{u}\right)^{l+1}} \sum_{j=0}^{\infty} \frac{\left(-i k_{o} \beta\right)^{l+j+1}\left(y-y_{1}\right)^{2 l+2 j+2}(\sqrt{t})^{2 j+2 l+2}}{(2 j+2 l+2) ! j !}
$$

and compare with $\mathcal{J}_{2 l+2}$ using

$$
\begin{array}{r}
\frac{2}{\left(i k_{o} \bar{u}\right)^{l+1}}\left(\frac{1}{t}\right)^{l+1} \sum_{j=0}^{\infty} \frac{\left(-i k_{o} \beta\right)^{l+j+1}\left(y-y_{1}\right)^{2 l+2 j+2}(\sqrt{t})^{2 j+2 l+2}}{(j+2 l+2) ! j !} \\
\quad=\frac{2}{\left(i k_{o} \bar{u}\right)^{l+1}}\left(\frac{1}{t}\right)^{l+1} \mathcal{J}_{2 l+2}\left(2 \sqrt{-i k_{o} \beta}\left(y-y_{1}\right) \sqrt{t}\right)
\end{array}
$$

where $\mathcal{J}_{2 l+2}$ is the Bessel function of first kind of order $2 l+2$.

For any fixed value of $l$,

$$
\begin{aligned}
& \left|\frac{2}{\left(i k_{o} \bar{u}\right)^{l+1}}\left(\frac{1}{t}\right)^{l+1} \frac{\left(-i k_{o} \beta\right)^{l+j+1}\left(y-y_{1}\right)^{2 l+2 j+2}(\sqrt{t})^{2 j+2 l+2}}{(2 j+2 l+2) ! j !}\right| \\
& \leq\left|\frac{2}{\left(i k_{o} \bar{u}\right)^{l+1}}\left(\frac{1}{t}\right)^{l+1} \frac{\left(-i k_{o} \beta\right)^{l+j+1}\left(y-y_{1}\right)^{2 l+2 j+2}(\sqrt{t})^{2 j+2 l+2}}{(j+2 l+2) ! j !}\right|
\end{aligned}
$$

Hence by the comparison test $g_{l}(y, t)$ is convergent since $\mathcal{J}_{2 l+2}$ is convergent.

Finally we observe that

$$
\phi_{1}(y, t)=\sum_{l=0}^{\infty} \frac{g_{l}(y, t)}{t^{l+1}}
$$

is convergent as $t \rightarrow \infty$. 


\section{Bibliography}

[1] Abramowitz, M., Stegun, I.A., 1964, Handbook of mathematical functions with formulas, graphs and mathematical tables, Nat. Bur. Stands, 1046 pp.

[2] Ablowitz, M.J., Fokas, A.S., 2003, Introduction and application of complex variables, Cambridge University Press, second edition.

[3] Batchelor, G.K., 1967, An introduction to fluid dynamics, Cambridge University Press, 615 pp.

[4] Béland, M., 1976, Numerical study of the nonlinear Rossby wave critical level development in a barotropic zonal flow, J. Atmos. Sci., 33, 2066-2078.

[5] Béland, M., Warn, T., 1975, The radiation condition for transient Rossby waves, J. Atmos. Sci., 32, 1873-1880.

[6] Booker, J.R., Bretherton, F.P., 1967, The critical layer for gravity waves in a shear flow, J. Fluid Mech., 27, 513-539.

[7] Campbell, L.J., 2004, Wave-mean flow interactions in a forced Rossby wave packet critical layer Stud. Appl. Math., 47, 39-85.

[8] Campbell, L.J., Maslowe, S.A., 1998, Forced Rossby wave packets in barotropic shear flows with critical layers, Dyn. Atmos. Oceans, 28, 9-37. 
[9] Campbell, L.J., Maslowe, S.A., 2003, Nonlinear critical-layer evolution of a forced gravity wave packet, J. Fluid Mech., 29, 151-179.

[10] Dickinson, R.E., 1970, Development of a Rossby wave critical level, $J$. Atmos. Sci., 27, 627-633.

[11] Holton, J.R., 2004, An introduction to dynamic meteorology, Academic Press, New York.

[12] Howard, L.N., 1961, Note on a paper of John W. Miles, J. Fluid Mech., 10, 509-512.

[13] Kundu, P.K., Cohen, I.M., 2004, Fluid mechanics, Academic Press, New York.

[14] Lin, C.C., 1955, The theory of hydrodynamic stability, Cambridge Univ. Press, Cambridge, U.K., 1955

[15] Miles, J.W., 1961, On the stability of heterogenous flows, J. Fluid Mech., 10, 496-508.

[16] Nadon, M., Campbell, L.J, 2007, An exact expression for transient forced internal gravity waves in a Boussinesq fluid, Wave motion, 6, 340-345.

[17] Nappo, C.J., 2002, An introduction to atmospheric gravity waves, Academic Press, New York.

[18] Pearson, R.A., 1974, Consistant boundary conditions for numerical models of systems that admit dispersive waves, J. Atmos. Sci., 9, 1481-1489.

[19] Pogany, T.K., Suli, E., 2009, Integral representation of Neumann series of Bessel functions, Proceedings of the A.M.S. Vol. 37, 2363-2368. 
[20] Spiegel, E.A., Veronis, G., 1960, On the Boussinesq approximation for compressible fluid, Astrophys. J., 6, 442-447.

[21] Warn, T., Warn, H., 1976, On the development of a Rossby wave critical level, J. Atmos. Sci., 33, 2021-2024.

[22] Warn, T., Warn, H., 1978, The evolution of a nonlinear critical level, Stud. Appl. Math., 59, 37-71.

[23] Warren, F.W.G., 1968, A note on Howard's proof of Mile's theorem, Quart. Journ. Mech. Applied Math., Vol. XXI, Pt. 4 